\title{
Statistical Methods for Signal Detection in Pharmacovigilance
}

\author{
By \\ Christopher Gravel \\ B.Math, B.A (Psychology)

\begin{abstract}
A thesis submitted to the Faculty of Graduate Studies and Research in partial fulfillment of the requirement for the degree of Master of Science
\end{abstract}

School of Mathematics and Statistics

Ottawa Carleton Institute for Mathematics and Statistics

\author{
Carleton University \\ Ottawa, Ontario
}

January, 2009

Copyright

2009 Chris Gravel C 


$\begin{array}{ll}\begin{array}{l}\text { Library and } \\ \text { Archives Canada }\end{array} & \begin{array}{l}\text { Bibliothèque et } \\ \text { Archives Canada }\end{array} \\ \begin{array}{l}\text { Published Heritage } \\ \text { Branch }\end{array} & \begin{array}{l}\text { Direction du } \\ \text { Patrimoine de l'édition }\end{array} \\ \begin{array}{l}\text { 395 Wellington Street } \\ \text { Ottawa ON K1A 0N4 } \\ \text { Canada }\end{array} & \begin{array}{l}\text { 395, rue Wellington } \\ \text { Ottawa ON K1A 0N4 } \\ \text { Canada }\end{array}\end{array}$

Your file Votre référence ISBN: 978-0-494-47534-8 Our file Notre référence ISBN: 978-0-494-47534-8

NOTICE:

The author has granted a nonexclusive license allowing Library and Archives Canada to reproduce, publish, archive, preserve, conserve, communicate to the public by telecommunication or on the Internet, loan, distribute and sell theses worldwide, for commercial or noncommercial purposes, in microform, paper, electronic and/or any other formats.

The author retains copyright ownership and moral rights in this thesis. Neither the thesis nor substantial extracts from it may be printed or otherwise reproduced without the author's permission.
AVIS:

L'auteur a accordé une licence non exclusive permettant à la Bibliothèque et Archives Canada de reproduire, publier, archiver, sauvegarder, conserver, transmettre au public par télécommunication ou par l'Internet, prêter, distribuer et vendre des thèses partout dans le monde, à des fins commerciales ou autres, sur support microforme, papier, électronique et/ou autres formats.

L'auteur conserve la propriété du droit d'auteur et des droits moraux qui protège cette thèse. $\mathrm{Ni}$ la thèse ni des extraits substantiels de celle-ci ne doivent être imprimés ou autrement reproduits sans son autorisation.
In compliance with the Canadian Privacy Act some supporting forms may have been removed from this thesis.

While these forms may be included in the document page count, their removal does not represent any loss of content from the thesis.
Conformément à la loi canadienne sur la protection de la vie privée, quelques formulaires secondaires ont été enlevés de cette thèse.

Bien que ces formulaires aient inclus dans la pagination, il n'y aura aucun contenu manquant.

\section{Canada}


Pharmacovigilance attempts to detect potential correlations between certain drugs and adverse reactions to these drugs through the analysis of spontaneous reporting data. A variety of mathematical methods are used in a signal detection style to search out these greater than expected pairs of drugs and adverse reactions. First, a theoretical examination of these methods as well as their properties and their place in the current pharmacovigilance literature is conducted. Second, the introduction of a method commonly used in categorical data analysis that has not been applied to pharmacovigilance is considered. Then, a data example considering the effects of Vioxx on hypertension will be looked at from the perspective of the method's abilities to flag this known interaction. Finally, a simulation study is conducted to examine the estimators in action. This is followed by a discussion on power and type one error of the different methods. 
I would like to thank Dr. Patrick Farrell and Dr. Daniel Krewski for their support. Your insight and guidance has been a huge help in the researching and writing of this thesis. I am extremely grateful for all they have done.

Also I'd like to thank the School of Mathematics and Statistics at Carleton University for training and teaching me the skills I needed to complete this project.

Finally, I'd like to thank my parents, Dr. Roy and Ying Gravel, for being there for me throughout my educational process. 
List of Formulas $\quad$ V

List of Diagrams vii

Chapter 1 Introduction 1

1.2 Objectives 4

1.3 Notation 5

$\begin{array}{lll}1.4 & \text { Mathematical Results } & 7\end{array}$

Chapter 2 Theoretical Discussion of Signal Detection Methods 9

- Frequentist Approach

2.1 Proportional Reporting Ratio 9

2.2 Reporting Odds Ratio and Yule's Q 22

2.3 Sequential Reporting Probability Ratio Test 33

Chapter 3 Theoretical Discussion of Signal Detection Methods 39

- Bayesian Approach

3.1 Bayesian Confidence Propogation Neural Network 40

3.2 Empirical Bayesian Screening 51

Chapter 4 Adjusted Pearson Residuals $\quad 65$

$\begin{array}{llr}\text { Chapter } 5 & \text { Data Example } & 69\end{array}$

$\begin{array}{lll}5.1 & \text { Results } & 73\end{array}$ 
$\begin{array}{lll}\text { Chapter } 6 & \text { Simulation Study } & 75\end{array}$

6.1 General Concerns 75

6.2 Methods 83

$\begin{array}{lll}6.3 & \text { Results } & 90\end{array}$

6.4 Comparison of Methods 93

$6.5 \quad$ Significance levels 95

6.6 Power Analysis 100

6.7 Empirical Bayes (Roux) 108

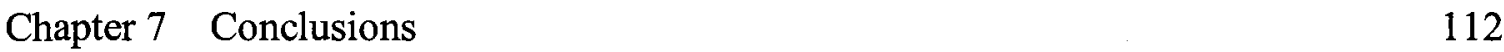

$\begin{array}{lll}\text { Appendix 1 Vioxx Data } & 117\end{array}$

$\begin{array}{lll}\text { Appendix } 2 \text { Simulation Study } & 121\end{array}$

$\begin{array}{ll}\text { References } & 155\end{array}$ 


\section{List of Formulas}

1.4.1 Second order multivariate Taylor series $\quad 7$

1.4.2 Second order bivariate Taylor series $\quad 8$

1.4.3 The Delta method $\quad 8$

2.1.1 The proportional reporting ratio 9

$\begin{array}{lll}2.1 .2 & E(\ln (P R R)) & 14\end{array}$

2.1.3 Yate's Chi-square 16

$\begin{array}{lll}2.1 .4 & V(\ln (P R R)) & 18\end{array}$

2.2.1 The reporting odds ratio $\quad 22$

2.2.2 $E(\ln (R O R)) \quad 23$

2.2.3 Asymptotic distribution of $\ln (R O R) \quad 28$

$\begin{array}{lll}2.2 .4 \quad V(\ln (R O R)) & 29\end{array}$

2.2.5 Yule's Q 29

$\begin{array}{lll}2.2 .6 & V(Q) & 30\end{array}$

2.3.1 The Sequential Probability Ratio Test using Roux's criteria 37

3.1.1 The Information Component 41

3.1.2 Approximate $E\left(I C_{i j}\right) \quad 45$

3.1.3 Approximate $V\left(I C_{i j}\right) \quad 46$

3.1.4 Expectation of a $\log$ Beta distributed random variable with $\quad 47$ parameters $\alpha, \beta$

3.1.5 Variance of a $\log$ Beta distributed random variable with parameters 48 $\alpha, \beta$

3.1.6 Exact $E\left(I C_{i j}\right) \quad 49$

3.1.7 Exact $V\left(I C_{i j}\right) \quad 49$

3.2.1 Definition of $\lambda_{i j}$ 
3.2.2 $f\left(\lambda_{i j} ; \alpha_{1}, \beta_{1}, \alpha_{2}, \beta_{2}, P\right)$

3.2.3 Marginal distribution of $n_{i j}$

3.2.4 Posterior weights $Q_{n}$

3.2.5 Posterior distribution $f\left(\lambda_{i j} \mid n_{i j}\right) \quad 56$

3.2.6 Posterior expectation $\lambda_{i j} \mid n_{i j}$

3.2.7 Posterior expectation of $\ln \left(\lambda_{i j}\right) \mid n_{i j}$

3.2.8 The Empirical Bayes Geometric Mean 59

3.2.9 Roux's criteria for signal classification 60

3.2.10 Signal classification using quantiles of the posterior distribution 64

4.1.1 Pearson Standardized Residuals 66

$\begin{array}{lll}\text { 4.1.2 Adjusted Pearson Residual } & 78\end{array}$

6.2.1 Weight function used in Lowess procedure 92 
1.3.1 IxJ Contingency table

1.3.2 2x2 Contingency table 6

$\begin{array}{lll}\text { 1.3.3 Alternate notation } & 6\end{array}$

5.1.1 Signals flagged for $2^{\text {nd }}$ quarter of 2004 in CDER database 72

5.1.2 Decisions made for Vioxx/Hypertension Pair 73

$\begin{array}{lll}\text { 6.1.1 Exposure to Drug } & 78\end{array}$

$\begin{array}{lll}\text { 6.1.2 Reporting Probability } & 79\end{array}$

6.1.3 Membership Function $\quad 80$

6.1.4 Values associated with the Membership Function 80

6.1.5 Membership Functions used in example $\quad 81$

6.2.1 Signal Detection Criteria at 5\% significance level 88

6.4.1 Power versus RR for the remaining methods at 5\% significance 93

6.6.1 Power comparison of remaining methods at 5\% significance 100

$\begin{array}{ll}\text { 6.6.2 Actual data for power comparison } & 101\end{array}$

6.6.3 Power comparison for ROR at 5\% significance across three types of $\quad 102$ variability

6.6.4 Power comparison with respect to underlying variability for Adjusted $\quad 103$ Residual and WHO approximation methods

6.6.5 Power comparison for ROR across three levels of significance 104

6.6.6 Power comparison for ROR of three significance levels within a 106 range of $\mathrm{RR}=[2,3]$

6.6.7 Power comparison for ROR of three significance levels within a 107 range of $R R=[2,3]$

6.7.1 Power versus RR for EBGM (Roux) across three types of underlying $\quad 110$ variability 


\section{Chapter 1 - Introduction}

\subsection{Introduction}

After pharmaceutical companies release their products into the marketplace, potentially dangerous drugs may go undetected until people start experiencing some of the adverse effects. Health agencies around the world have responded to this problem by instituting data collection systems that will enable statisticians to study this problem. The study of this data and development of the appropriate statistical tools is known as pharmacovigilance.

These data gathering systems are known as Spontaneous Reporting Systems (SRS). The concept of spontaneous reporting is that individuals will report their adverse drug reactions (ADRs) to a variety of sources when seeking out help. These sources range from the pharmacists and doctors to the individual directly reporting the side effect to the appropriate health agency. The reports can appear in a variety of ways but the basic similarities between these reports include the name(s) of the drug(s) being taken by the individual and the $\mathrm{ADR}(\mathrm{s})$ being experienced. The number of times a particular ADR is reported for a given drug is recorded. Since this information is obtained for many different ADR-drug combinations, the result is a database that is reflected by a large dimensional contingency table of counts. 
This data holds a great number of difficulties for the analyst attempting to weed out the significantly large interactions between drug and ADR. First of all, there is a certain level of exposure of ADRs in the table that have nothing to do with drugs. For example, there will be a certain level of heart attacks, based purely on the 'natural' occurrence of heart attacks and not due to taking a given drug. Determining the causal properties of an entry in the table is impossible. Thus, the pharmacovigilance experts have focused their interest on signal detection methods that will point out potential problem drugs and leave the causality discussion to the pharmacologists.

Another issue is the difficulty of multi-item interactions. Individuals taking multiple drugs that experience a particular ADR will be recorded in this database as an entry for each drug associated with the given ADR. However it is extremely difficult to tell whether the ADR is a result of an individual drug, all of the drugs or even a subset of the drugs being taken. The opposite is true as well in that multiple ADRs can confound the pairwise correlation and muffle a specific drug/ADR interaction. This reinforces the decision to examine this data from the perspective of simply pointing out potentially dangerous interactions and allowing the pharmacologists to further research the drugs. For the purposes of this review, we will not consider interactions based on multiple sources and only look at the data created by a direct comparison of a drug and an ADR.

A number of statistical methods have been proposed to analyze SRS datasets. Due to the nature of the data a number of these methods are taken from standard techniques in categorical data analysis, specifically those that test independence of 
variables. Some of the methods include the Proportional Reporting Ratio (PRR), the Reporting Odds Ratio (ROR) and Yule's Q. The first of these approaches is effectively a measure of relative risk, while the ROR mimics an odds ratio. Significance tests are performed by assuming a distribution to the counting variable and then determining a critical value for comparison. This variable is commonly assigned to be a Poisson random variable. Also, applying a simple transformation to the random variable in question allows us to consider Yate's Chi-square as another decision making technique. Sequential tests are another method of signal detection used in pharmacovigilance. Through updating a statistic with new data, we can observe whether the statistic crosses predetermined boundaries created through the weighting of Type 1 and Type 2 errors. These errors represent respectively, the probability of rejecting the null hypothesis under the condition that it is true and the probability of rejecting the alternative hypothesis under the condition that it is true. In our discussion, assuming a null hypothesis of no signal, a Type 1 error would be the probability that a signal is flagged given that there is actually no signal present, and a Type 2 error would be the probability of concluding no signal when in fact one exists. A method not currently used in pharmacovigilance that we propose and discuss here, is a standardized Pearson residual. Finally, two Bayesian methods are considered, namely the Bayesian Confidence Propogation Neural Network (BCPNN) or WHO method and empirical Bayes Screening (EBS). 


\subsection{Objectives}

This thesis is broken down as follows. In Chapters 2 and 3, the signal detection methods listed above will be discussed through a thorough examination of their theoretical properties as well as a discussion on the pros and cons of these methods. Specifically, derivations of the expectations and variances of these estimators will be considered as well as an examination of their application to SRS data. Some of the practical issues aiding or hindering these methods in the search for signals will also be

discussed. Chapter 4 will introduce the Pearson adjusted residual for use in pharmacovigilance, again through a theoretical review of the estimators properties. Then, a practical example to demonstrate the estimators in action is presented in Chapter 5 . In Chapter 6 the results of a simulation study, which was conducted for the purpose of comparing the methods through a power analysis, will be discussed. In Chapter 7 , we conclude with a discussion of the circumstances in which each method best performs and talk about the directions for future research.

Finally, a number of problems in the pharmacovigilance literature are brought up for discussion throughout this paper. The first is the formula for the logarithmic variance of the PRR. Another problematic formula is found in the literature on the empirical Bayes method when looking at the logarithmic variance of the posterior distribution. Finally, the simulation study conducted in Chapter 6 demonstrates that some of these methods have difficulty holding the significance level at a preset value largely due to issues regarding the chosen critical values in the literature. 


\subsection{Notation}

To facilitate the discussion of signal detection methods, we consider a set of $J$ adverse reactions to $I$ different drugs within a specified time period. The resulting data can be summarized in an $I \times J$ contingency table:

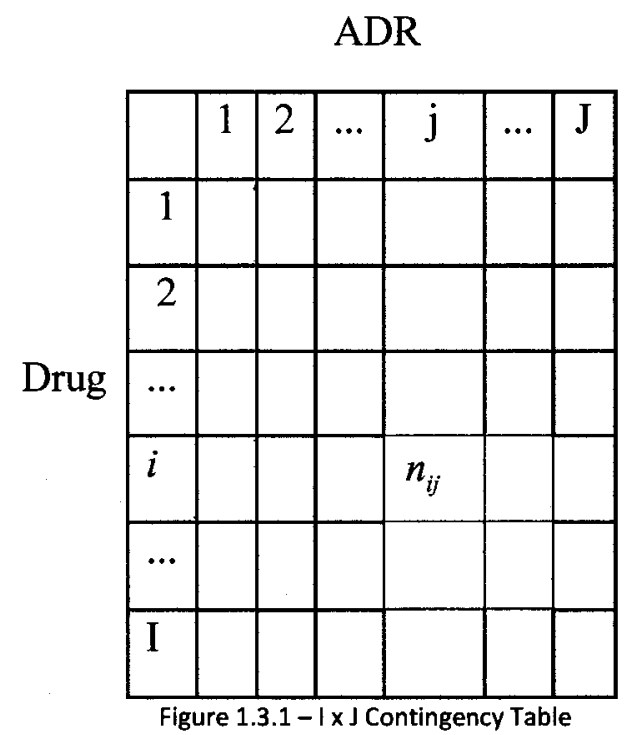

Here, $n_{i j}$ represents the number of times the $j^{\text {th }}$ ADR, $A_{j}$ is reported for the $i^{\text {th }}$ drug, $D_{i}$. To illustrate the use of the different signal detection methods, it suffices to reduce this $I \times J$ contingency table to a $2 \times 2$ table which aggregates the data for all drugs with the exception of drug $D_{i}$, and all ADRs with the exception of $A_{j}$. This yields the more compact contingency table with notation denoted as: 


\begin{tabular}{|c|c|c|c|}
\hline & $A_{j}$ & $A_{j}^{C}$ & Total \\
\hline $\begin{array}{c}\text { Drug } \\
D_{i}\end{array}$ & $n_{i j}$ & $n_{i .}-n_{i j}$ & $n_{i .}$ \\
\hline $\begin{array}{c}\text { Other } \\
\text { Drugs }\end{array}$ & $n_{. j}-n_{i j}$ & $n_{. .}-n_{. j}-n_{i .}+n_{i j}$ & $n_{. .}-n_{i .}$ \\
$D_{i}^{C}$ & & & \\
\hline Total & $n_{. j}$ & $n_{. .}-n_{. j}$ & $n_{. .}$ \\
\hline
\end{tabular}

Figure 1.3.2-2 $\times 2$ Contingency Table

Here, $n_{i .}$ is the total number of ADR reports for drug $D_{i}$ so that $n_{i .}=\sum_{j} n_{i j}, n_{. j}$ is the number of reports of the $j^{t h}$ ADR, $A_{j}$, across all drugs so that $n_{. j}=\sum_{i} n_{i j}$, and $n_{. .}$is the total number of reports of all ADR's across all drugs, thus $n_{. .}=\sum_{i j} n_{i j}$. Note that the set of drugs included in the contingency table will involve some expert judgement on the part of experienced investigators familiar with commonalties and differences among the set of drugs under consideration.

Finally, an alternative notation commonly used in the pharmacovigilance literature, will at times be used in this discussion especially when proving results. It will be referred to in the text as notation (1.3.3). For this notation let $A=n_{i j}, B=n_{i .}-n_{i j}, C=n_{. j}-n_{i j}$ and $D=n_{. .}-n_{. j}-n_{i .}+n_{i j}$. Whenever this switch is made, it will be noted in the text. 


\subsection{Mathematical Results}

Throughout this paper a variety of mathematical results are used while proving the various properties of the estimators used in signal detection methods. The following is a list of these results.

Second order multivariate Taylor series about $\mathbf{P}=\mathbf{p}$ where $\mathbf{P}=\left(P_{1}, \ldots, P_{n}\right)$ and $\mathbf{p}=\left(p_{1}, \ldots, p_{n}\right)$

$$
\begin{aligned}
& f(\mathbf{P}) \approx f(\mathbf{p})+\Delta(\mathbf{p})(\mathbf{P}-\mathbf{p})+\frac{(\mathbf{P}-\mathbf{p}) \mathrm{H}(\mathbf{p})(\mathbf{P}-\mathbf{p})^{T}}{2} \\
& \text { where } \Delta(\mathbf{p})=\left\{\left.\frac{\partial f}{\partial P_{1}}\right|_{P_{1}=p_{1}}, \ldots,\left.\frac{\partial f}{\partial p_{n}}\right|_{P_{n}=p_{n}}\right\} \text { and } \\
& \mathrm{H}(\mathbf{p})=\left(\begin{array}{cccc}
\left.\frac{\partial^{2} f}{\partial P_{1}^{2}}\right|_{P_{1}=p_{1}} & \left.\frac{\partial^{2} f}{\partial P_{1} \partial P_{2}}\right|_{\substack{P_{1}=p_{1} \\
P_{2}=p_{2}}} & \cdots & \left.\frac{\partial^{2} f}{\partial P_{1} \partial P_{n}}\right|_{\substack{P_{1}=p_{1} \\
P_{n}=p_{n}}} \\
\left.\frac{\partial^{2} f}{\partial P_{2} \partial P_{1}}\right|_{\substack{P_{1}=p_{1} \\
P_{2}=p_{2}}} & \left.\frac{\partial^{2} f}{\partial P_{2}^{2}}\right|_{P_{2}=p_{2}} & & \cdots \\
\cdots & \cdots & & \left.\frac{\partial^{2} f}{\partial P_{n}^{2}}\right|_{P_{n}=p_{n}}
\end{array}\right)
\end{aligned}
$$

This equation will reduce to the following expression in the bivariate case:

Second order Bivariate Taylor Series Expansion about $X=x, Y=y$ 


$$
f(X, Y) \approx f(x, y)+\left.\frac{\partial f(X, Y)}{\partial X}\right|_{X=x, Y=y}(X-x)+\left.\frac{\partial f(X, Y)}{\partial Y}\right|_{X=x, Y=y}(Y-y)
$$

$+\left.\frac{1}{2} \frac{\partial^{2} f(X, Y)}{\partial X^{2}}\right|_{X=x, Y=y}(X-x)^{2}+\left.\frac{1}{2} \frac{\partial^{2} f(X, Y)}{\partial Y^{2}}\right|_{X=x, Y=y}(Y-y)^{2}+\left.\frac{\partial^{2} f(X, Y)}{\partial X \partial Y}\right|_{X=x, Y=y}(X-x)(Y-y)$

The delta method

Let $\mathbf{p}=\left(p_{1}, \ldots, p_{n}\right)^{T}$ be a random vector that is asymptotically multivariate normal with mean $\boldsymbol{\mu}=\left(\mu_{1}, \ldots, \mu_{n}\right)^{T}$ and variance $. \frac{\boldsymbol{\Sigma}}{n}, \boldsymbol{\Sigma}=\left\{\operatorname{Cov}\left(p_{i}, p_{j}\right)\right\}_{i, j=1}^{n}$

Let $g(\cdot)$ be a differentiable function with $\varphi=\left(\phi_{1}, \ldots, \phi_{n}\right)$ such that $\phi_{i}=\left.\frac{\partial g\left(p_{i}\right)}{\partial p_{i}}\right|_{\mu_{i}}$, then

$$
\sqrt{n}(g(\mathrm{p})-g(\mu)) \stackrel{d}{\longrightarrow} N\left(0, \varphi^{T} \Sigma \varphi\right.
$$




\section{Chapter 2}

Theoretical Discussion of Signal Detection Methods - Frequentist Approach

The discussion of signal detection methods can be expressed in a hypothesis testing scenario where the null hypothesis reflects a lack of association between drug and ADR. Since the $(i, j)^{\text {th }}$ cell can be represented as a joint probability of the presence of drug $D_{i}$ and ADR $A_{j}$ in the table, the testing problem can be described as $H_{0}$ : no signal versus $H_{1}$ : signal (2.1) where a signal can be described as an association larger than expected.

\subsection{Proportional Reporting Ratio}

The proportional reporting ratio (PRR) has been used by a number of authors (Evans $e t$ al., 2001; van Puijenbroek et al., 2002; Waller et al., 2004; Roux et al., 2005) may be written as:

$$
P R R=\frac{\frac{n_{i j}}{n_{i .}}}{\frac{n_{. j}-n_{i j}}{n_{. .}-n_{i .}}}
$$

The numerator is the number of observed interactions of a specific ADR, $A_{j}$, with a specific drug, $D_{i}$, compared to the number of observed interactions of any ADR with 
drug $D_{i}$. In other words, the PRR is a proportional measure of the frequency of adverse reactions $A_{j}$ within the set of all ADR's, given drug $D_{i}$. The denominator essentially compares the prevalence of $A_{j}$ across all other drugs excluding $D_{i}$ with the interactions of all other ADRs (excluding $A_{j}$ ) with all other drugs (excluding $D_{i}$ ). This estimator may be viewed as the ratio of conditional probabilities of observing an interaction between drug $D_{i}$ and ADR $A_{j}$ of the drug in question versus all other drugs. The PRR involves several assumptions. First, relative risk is only a valid measure in a prospective study or in a scenario where the row totals are fixed. In our application, we are examining the likelihood of $\operatorname{ADR} j$ with drug $i$, thus if we assume that we have knowledge of the prevalence of use for each drug in our table, then the PRR becomes a valid indicator for further discussion.

It is important to discuss the behaviour of the PRR when the relationship between the drug and ADR is an independent one, $H_{0}$, or when the influence of drug $D_{i}$ has no effect on the corresponding ADR, $A_{j}$. If the relative frequency of $A_{j}$ observed with $D_{i}$ is the same as the relative frequency of $A_{j}$ with all other drugs, we can then argue that the $A_{j} / D_{i}$ interaction is approximately equal to the relative frequency of this $\mathrm{ADR}$ due to chance alone. Under this condition the PRR should be one, since the numerator and denominator would be the same. However, if the numerator is larger than the denominator, the relative frequency of this specific ADR is greater than the relative frequency due to chance yielding a PRR greater than one and a signal is hypothesized. 
Since this estimator is subject to sampling variability, it is important to examine the mathematical expectation and variance as they relate to signal detection.

Note that this concept of fixing the row totals implies that the distribution of each fraction is a Binomial with the appropriate parameters. Specifically, $n_{i j} \sim \operatorname{Binomial}\left(n_{i .}, n_{i j} / n_{i .}\right)$ and $n_{. j}-n_{i j} \sim \operatorname{Binomial}\left(n_{. .}-n_{i .}, n_{. j}-n_{i j} / n_{. .}-n_{i .}\right)$. This means that we are dealing with a ratio of binomial random variables, and as such this is not a linear function. Therefore the expression for the expectation of this estimator under $H_{0}$ requires some level of approximation. Using a Taylor series we can get an idea of the true nature of this approximation.

Let $A=n_{i j}, B=n_{i .}-n_{i j}, C=n_{. j}-n_{i j}$ and $D=n_{. .}-n_{. j}-n_{i .}+n_{i j}$ then $A \sim \operatorname{Binomial}\left(a+b, \frac{a}{a+b}\right)$ and $C \sim \operatorname{Binomial}\left(c+d, \frac{c}{c+d}\right)$

Thus, $P R R=\frac{\frac{A}{A+B}}{\frac{C}{C+D}}=f(A, C)$. Applying a bivariate Taylor series expansion (1.4.2) about $A=a, C=c$ and the taking the expectation yields:

$E(f(A, C)) \approx f(a, c)+\left.\frac{1}{2} \frac{\partial^{2} f(A, C)}{\partial A^{2}}\right|_{A=a, C=c} \operatorname{Var}(A)+\left.\frac{1}{2} \frac{\partial^{2} f(A, C)}{\partial C^{2}}\right|_{A=a, C=c} \operatorname{Var}(C)$ because $E(A-a)=0, E(C-c)=0$ and because $A$ is independent of $C$ under $H_{0}, \operatorname{Cov}(A, C)=0$. 
The necessary partial derivatives are as follows:

$\left.\frac{\partial^{2} f(A, C)}{\partial A^{2}}\right|_{A=a, C=c}=\left.\frac{-2 b(c+d)}{c(a+b)^{3}} \quad \frac{\partial^{2} f(A, C)}{\partial C^{2}}\right|_{A=a, C=c}=\frac{2 a d}{c^{3}(a+b)}$

Thus, $E(P R R) \approx \frac{a(c+d)}{c(a+b)}+\frac{1}{2} \frac{-2 b(c+d)}{c(a+b)^{3}}(a+b)\left(\frac{a}{a+b}\right)\left(\frac{b}{a+b}\right)$

$$
\begin{gathered}
+\frac{1}{2} \frac{2 a d}{c^{3}(a+b)}(c+d)\left(\frac{c}{c+d}\right)\left(\frac{d}{c+d}\right) \\
\approx \frac{a(c+d)}{c(a+b)}+\frac{a c d^{2}}{c^{3}(a+b)(c+d)}-\frac{a b^{2}(c+d)}{c(a+b)^{4}} .
\end{gathered}
$$

Therefore the PRR's bias is expressed as $\frac{a d^{2}}{c^{2}(a+b)(c+d)}-\frac{a b^{2}(c+d)}{c(a+b)^{4}}$, or in notation (1.3.2), $\frac{n_{i j}\left(n-n_{. j}-n_{i .}+n_{i j}\right)^{2}}{n_{i .}\left(n-n_{i .}\right)\left(n_{. j}-n_{i j}\right)^{2}}-\frac{n_{i j}\left(n_{i .}-n_{i j}\right)^{2}\left(n . .-n_{i .}\right)}{n_{i .}^{4}\left(n_{. j}-n_{i j}\right)}$.

To determine whether the bias will affect the performance of the $P R R$ we must consider the quantity as it approaches infinity. To do so we will return to our alternate notation (1.3.3). First of all, note that the second term is dominated by the $(a+b)^{4}$ in the denominator implying that we can ignore this term as it will approach zero as $(a+b) \rightarrow \infty$. However, for the first term, it is not so clear. To determine its behaviour as it approaches infinity, we will examine the asymptotic behaviour with respect to each quantity in the $2 \times 2$ table. 
For $b$ we get

$$
\begin{aligned}
& \lim _{b \rightarrow \infty} \frac{a d^{2}}{c^{2}(a+b)(c+d)}=\lim _{b \rightarrow \infty} \frac{a d^{2}}{a c^{3}+a c^{2} d+b c^{3}+b c^{2} d} \\
& =\lim _{b \rightarrow \infty} \frac{a d^{2}}{a c^{3}+a c^{2} d+b c^{3}+b c^{2} d}=\frac{0}{c^{3}+c^{2} d}=0 .
\end{aligned}
$$

Similarly, the limit as $c \rightarrow \infty$ is also zero.

$$
\begin{aligned}
& \lim _{c \rightarrow \infty} \frac{a d^{2}}{c^{2}(a+b)(c+d)}=\lim _{c \rightarrow \infty} \frac{a d^{2}}{a c^{3}+a c^{2} d+b c^{3}+b c^{2} d} \\
& =\lim _{c \rightarrow \infty} \frac{a d^{2}}{a c^{3}+a c^{2} d+b c^{3}+b c^{2} d}=\frac{0}{a+b}=0 .
\end{aligned}
$$

When $a \rightarrow \infty$ we have a problem:

$\lim _{a \rightarrow \infty} \frac{a d^{2}}{c^{2}(a+b)(c+d)}=\lim _{a \rightarrow \infty}\left(\frac{a}{a+b}\right)\left(\frac{d^{2}}{c^{3}+c^{2} d}\right)=\frac{d^{2}}{c^{3}+c^{2} d}$. Thus this limit relies on the behaviour of the second row of our $2 \times 2$ table, or the set of other drugs. Specifically, when $a$ is large, if $d^{2} \geq c^{3}+c^{2} d$ the bias will become large enough to cause issues with estimation.

Finally, the behaviour as $d \rightarrow \infty$ is $\lim _{d \rightarrow \infty} \frac{a d^{2}}{a c^{3}+a c^{2} d+b c^{3}+b c^{2} d}=\frac{a}{0}=\infty$.

This implies that as the number of times counts are observed between the reference set of drugs and ADRs increases, the bias will get larger and larger.

The consequences of a noticeable bias can be seen in the observed values of the estimator since it will shift in the positive direction causing misidentification of signals. Also the 
bias can affect the MSE increasing the variability causing potential signals to be missed. Thus, a biased PRR can cause numerous Type 1 and Type 2 errors. However the bias will generally converge to zero in our application because $a$ is the smallest value in the contingency table. In order for $a$ to outweigh $c$ the sample size of the drug in question has to be greater than $50 \%$ of the overall sample size and under this condition other more serious problems would arise as well.

Alternatively, we can take a logarithmic transformation to determine the expectation under $H_{0}$ which is the commonly used method in the literature.

$\ln (P R R)=\ln \left(\frac{\frac{n_{i j}}{n_{i .}}}{\frac{n_{. j}-n_{i j}}{n_{. .}-n_{i .}}}\right)=\ln \left(\frac{n_{i j}}{n_{i .}}\right)-\ln \left(\frac{n_{. j}-n_{i j}}{n_{. .}-n_{i .}}\right)$. Thus,
$E(\ln (P R R))=E\left(\ln \left(\frac{n_{i j}}{n_{i .}}\right)\right)-E\left(\ln \left(\frac{n_{. j}-n_{i j}}{n_{. .}-n_{i .}}\right)\right) \approx \ln \left(E\left(\frac{n_{i j}}{n_{i .}}\right)\right)-\ln \left(E\left(\frac{n_{. j}-n_{i j}}{n_{. .}-n_{i .}}\right)\right)$

$$
E(\ln (P R R)) \approx \ln \left(\frac{n_{i j}}{n_{i .}}\right)-\ln \left(\frac{n_{. j}-n_{i j}}{n_{. .}-n_{i .}}\right) .
$$

This quantity is asymptotically unbiased, thus the taking a logarithmic transformation of this estimator allows us to ignore the preceding discussion regarding the bias.

Of note is the fact that the PRR depends on quantities other than the cell counts, $n_{i j}$, in question. In particular, the denominator depends to a great extent on the size of $n_{i}-n_{i j}$. Thus, if drug $D_{i}$ is associated with one specific ADR the rest of the observed 
PRRs for drug $D_{i}$ will be diminished due to an inflated denominator alternatively, if $n_{i}-n_{i j}$ is extremely large due to a single drug/ADR interaction, the quantity $\left(n_{. j}-n_{i j}\right) n_{i .}$ will be extremely large and will cause the value of the PRR to be unduly small. This also applies if there is a large value for $n_{. j}-n_{i j}$ due to another drug's association with the event $A_{j}$, causing the value of $n_{. j}-n_{i j}$ and the value of the PRR to be unduly inflated. To deal with this unwarranted bias in the numerator of the PRR, the original proponents assert that one can simply remove a large cell from the calculation of the PRR and continue as normal.

Now that we have examined the expectation of the PRR and have discussed some of the estimators' problems we can begin to look at how the estimator is applied to signal detection. To attempt to deal with sampling variability, some authors (Evans et al., 2001) have combined the use of the PRR with a commonly used method of assessing pairwise dependency among the variables found in a contingency table. This method is the Yate's Chi-squared test, which is essentially a discrete version of Pearson's Chi-square. Pearson's Chi-square measures the level of dependency between variables and is represented by the following statistic: $\chi_{P}^{2}=\sum_{i=1}^{n} \frac{\left(O_{i}-E_{i}\right)^{2}}{E_{i}}$. Here $O_{i}$ represents an observed frequency and $E_{i}$ represents the expected frequency of a variable of interest. Note that the statistic is essentially the square of a standard normal random variable, which is the justification for the Chi-square distribution. Yate's showed (Yates, 1934) 
that subtracting a half from the deviation in the numerator will greatly improve the approximations in a $2 \times 2$ table when the data is categorically distributed. Thus, our statistic takes on the form:

$$
\chi_{o}^{2}=\sum_{i=1}^{2} \sum_{j=1}^{2} \frac{\left(\left|n_{i j}-E_{i j}\right|-0.5\right)^{2}}{E_{i j}}
$$

such that $E_{i j}=\frac{n_{i \cdot} n_{. j}}{n_{.}}$. To apply this to our current application, this statistic is often compared to the $95^{\text {th }}$ percentile of a chi-square distribution with one degree of freedom (a numerical value of 3.84) to determine if there is evidence of an association between drug utilization and the ADR.

The authors (Evans et al., 2001) identify a signal as PRR greater than 2, a cell count greater than 3, and an observed chi-square statistic with Yate's correction $>4$, since the probability of observing a chi-square random variable with one degree of freedom taking on a value of 4 or more is extremely small. Using a PRR $>2$ as evidence of a signal allows for random fluctuation around the null value of one, while acknowledging that a PRR of less than one is definitely not a signal. This makes intuitive sense, since we are comparing the $\mathrm{ADR}$ occurrence rate for a specific drug with the occurrence rate of the same ADR across all other drugs.

Other authors (van Puijenbroek et al., 2002) have approached signal detection with the PRR from a more classical statistical inference approach. Due to the fact that 
the raw formula for this estimator is obviously dependent on the data it is possible to get some highly variable values for the PRR. For example, if the denominator gets extremely small, the PRR will become extremely large and vice versa. Therefore it is useful to continue our discussion of the mathematical properties and develop an expression for the variance and subsequently a confidence interval for the PRR. Since the PRR's distribution is highly skewed we cannot simply label it normally distributed and calculate a general interval based on this assumption. Instead we consider the distribution of the natural logarithm of the PRR, since it will possess a more bell-shaped and symmetric distribution. Using the delta method we can get a concrete expression for this asymptotic variance.

Let $\quad p_{\text {Drug }}=\frac{n_{i j}}{n_{i .}} \quad p_{\text {Other }}=\frac{n_{. j}-n_{i j}}{n_{. .}-n_{i .}}$

Then $P R R=\frac{p_{\text {Drug }}}{p_{\text {Other }}}$ and $\ln (P R R)=\ln \left(p_{\text {Drug }}\right)-\ln \left(p_{\text {Other }}\right)$

such that $\quad n_{i j} \sim \operatorname{Binomial}\left(n_{i .}, p_{\text {Drug }}\right)$

$$
n_{. j}-n_{i j} \sim \operatorname{Binomial}\left(n-n_{i .}, p_{\text {other }}\right)
$$

Then $\operatorname{Var}\left(n_{i j}\right)=n_{i .}\left(p_{\text {Drug }}\right)\left(1-p_{\text {Drug }}\right) \quad \operatorname{Var}\left(n_{. j}-n_{i j}\right)=\left(n_{. .}-n_{i .}\right)\left(p_{\text {Other }}\right)\left(1-p_{\text {Other }}\right)$

And $\operatorname{Var}\left(\hat{p}_{\text {Drug }}\right)=\frac{p_{\text {Drug }}\left(1-p_{\text {Drug }}\right)}{n_{i .}} \quad \operatorname{Var}\left(\hat{p}_{\text {Other }}\right)=\frac{p_{\text {Other }}\left(1-p_{\text {Other }}\right)}{n_{. .}-n_{i .}}$

Finally, $\quad \operatorname{Var}(\ln (P R R))=\operatorname{Var}\left(\ln \left(p_{\text {Drug }}\right)\right)+\operatorname{Var}\left(\ln \left(p_{\text {Other }}\right)\right)$ 
Using the Delta Method (1.4.3),

$$
\begin{gathered}
\operatorname{Var}\left(\ln \left(p_{\text {Drug }}\right)\right) \approx\left[\frac{\partial \ln \left(p_{\text {Drug }}\right)}{\partial p_{\text {Drug }}}\right]^{2} \operatorname{Var}\left(\hat{p}_{\text {Drug }}\right)=\frac{1}{p_{\text {Drug }}^{2}} \frac{p_{\text {Drug }}\left(1-p_{\text {Drug }}\right)}{n_{i .}} \\
=\frac{1-p_{\text {Drug }}}{n_{i .} p_{\text {Drug }}} \\
\begin{aligned}
\operatorname{Var}\left(\ln \left(p_{\text {Other }}\right)\right) \approx\left[\frac{\partial \ln \left(p_{\text {Other }}\right)}{\partial p_{\text {Other }}}\right]^{2} \operatorname{Var}\left(\hat{p}_{\text {Other }}\right) & =\frac{1}{p_{\text {Other }}^{2}} \frac{p_{\text {Other }}\left(1-p_{\text {Other }}\right)}{\left(n-n_{i .}\right)} \\
& =\frac{1-p_{\text {Other }}}{\left(n-. . n_{\text {i. }}\right) p_{\text {Other }}}
\end{aligned}
\end{gathered}
$$

Therefore,

$$
\begin{aligned}
\operatorname{Var}(\ln (P R R)) \approx & \frac{1-p_{\text {Drug }}}{n_{i .} p_{\text {Drug }}}+\frac{1-p_{\text {Oher }}}{\left(n-n_{i .}\right) p_{\text {Other }}}= \\
& \frac{1}{n_{i .} p_{\text {Drug }}}-\frac{p_{\text {Drug }}}{n_{i .} p_{\text {Drug }}}+\frac{1}{\left(n_{. .}-n_{i .}\right) p_{\text {Other }}}-\frac{p_{\text {Other }}}{\left(n-.-n_{i .}\right) p_{\text {Other }}}
\end{aligned}
$$

By substituting

$$
p_{\text {Drug }}=\frac{n_{i j}}{n_{i .}} \quad p_{\text {Other }}=\frac{n_{. j}-n_{i j}}{n_{. .}-n_{i .}} \quad \text { we get }
$$

$$
V(\ln (P R R)) \approx \frac{1}{n_{i j}}-\frac{1}{n_{i .}}+\frac{1}{n_{. j}-n_{i j}}-\frac{1}{n_{. .}-n_{i .}}
$$

The derivation of this variance formula is in agreement with that for similar estimators, such as the logarithmic variance of the Relative Risk (RR) (Agresti, 2002) 
and the logarithmic variance of the Incidence Rate Ratio (IRR) (Greenland and Rothman, 2008). However, it seems that the logarithmic variance of the PRR is frequently expressed as

$$
V(\ln (P R R))=\frac{1}{n_{i j}}-\frac{1}{n_{i .}}+\frac{1}{n_{. j}-n_{i j}}+\frac{1}{n_{. .}-n_{i .}}
$$

in the pharmacovigilance literature (van Puijenbroek et al., 2002; Roux et al., 2005), which is inconsistent with the expression derived above. However, due to the nature of the data considered here, the difference in the two formulas is negligible. In order for a serious difference to be observed when the term $\left(n-n_{i}\right)^{-1}$ is added instead of subtracted, its' value needs to be large enough to have a significant impact on the sum total of all terms. However, since $n=\sum_{i=1}^{n} n_{i .}$, in order for $n_{. .}-n_{i .}$ to be small, one particular drug must account for a large proportion (approximately 35 to 50\%) of the total sample size, which rarely occurs in pharmacovigilance. Finally, in exceptional cases where this term could have a noticeable impact in a particular application, the effect of using the latter formula will be a wider confidence interval. It follows that the variance formula presented here will enjoy stronger signal detection properties, reducing the likelihood of a false negative.

We can now create a $95 \%$ confidence interval for the natural logarithm of the proportional reporting ratio as follows: 


$$
\ln (P R R) \pm 1.96 \sqrt{\frac{1}{n_{i j}}-\frac{1}{n_{i .}}+\frac{1}{n_{. j}-n_{i j}}-\frac{1}{n_{. .}-n_{i .}}}
$$

Since we have taken the natural logarithm of the PRR, the null value of $\ln (P R R)$ is zero; as such, we can comfortably place our cut off for signal detection at a lower bound of zero. Exponentiating the confidence limits for $\ln (P R R)$ will provide (asymmetric) confidence limits for PRR.

An obvious issue with the PRR is how to deal with a cell count of zero, which would not only yield a PRR of zero, but the variance for $\ln (P R R)$ would be indeterminate. Zero cell counts occur frequently in pharmacovigilance data. This issue can be addressed by omitting all cells with zero counts in the calculation of the PRR; this is reasonable, since a cell count of zero is definitely not a signal of an adverse drug reaction.

Another issue was pointed out by Rothman et al (Rothman et al., 2004). They formulate an example involving interactions of a single drug with three different ADRs (ADR1, ADR2, and ADR3), where the purpose of the drug is to reduce the risk of ADR1. However, the PRR signals an interaction between this drug and ADR2. They then assign a fixed value for $n$ and proceed to evaluate the relative risk for all of the interactions. Curiously, the risk ratios for ADR2 do not yield a value that would imply a signal indicating that the PRR does not always successfully evaluate the risk of an interaction. 
Despite these limitations, the PRR also possesses some advantages as a tool for signal detection. First, the value of the PRR is proportional to the ratio of the rates of occurrences of ADRs, so that a larger PRR implies a larger signal. It follows that the PRR's value in signal detection is somewhat proportional to the strength of signal. The second major advantage of the PRR is that the values required can be taken directly from the data matrix and no extraneous information is needed. This is advantageous, since the nature of the data is such that the reporting rate of certain drug-ADR pairs will be biased by extraneous influences. The existence of reporting bias can limit the usefulness of an estimator, since avoidance of this bias requires outside knowledge of the attributes of the population under study. The only drawback to this advantage is that its base assumption requires that the reporting bias for the $\mathrm{ADR}$ in question be similar in magnitude to the reporting bias across all other ADRs. Fortunately, this assumption may be reasonable in most cases. 


\subsection{ROR and Yule's Q}

The PRR is essentially a measure of relative risk, comparing conditional probabilities of successfully observing interactions between drug and ADR under different circumstances. These probabilities reflect the likelihood of observing a specific ADR with drug $D_{i}$, and the chance of observing that same ADR across all other drugs in the dataset. One criticism of using this form of estimator is that it is based on cross-sectional data, for which indicators of relative risk are inappropriate (Agresti, 2002). A more appropriate measure is the Odds Ratio (OR), or, as it's termed in pharmacovigilance, the Reporting Odds Ratio (ROR). This measure reflects the magnitude of association between drug and ADR by comparing the odds of each possible outcome in the $2 \times 2$ table summarizing the available pharmacovigilance data given above. Note that the definition of odds here is the ratio of the probability of success to the probability of failure. Thus the ROR can be defined as:

$$
R O R=\frac{\frac{n_{i j}}{n_{i .}} / \frac{n_{i .}-n_{i j}}{n_{i .}}}{\frac{n_{. j}-n_{i j}}{n_{. .}-n_{. j}} / \frac{n_{. .}-n_{. j}-n_{i .}+n_{i j}}{n_{. .}-n_{. j}}}=\frac{n_{i j}\left(n-n_{. j}-n_{i .}+n_{i j}\right)}{\left(n_{. j}-n_{i j}\right)\left(n_{i .}-n_{i j}\right)}
$$

Once again, it is important to consider the mathematical properties of this estimator for the purposes of signal detection. Intuitively, the ROR has an expectation of one under $H_{0}$, or when there is no association between drug and ADR, which makes sense given the definition of the ROR. If there is a signal at a specific drug-ADR pair, then the 
ROR will be greater than one, since the interaction of interest is showing a higher probability of association than the other possible interactions (i.e., the ADR with all other drugs). If the ROR is less than one the magnitude of association is weighted more towards the other drugs than the drug under scrutiny implying no signal.

Similarly to the PRR, the ROR is a ratio of random variables, and as such any expression for the expectation will be an approximation. Here the only fixed value is $n_{n}$, or the grand total of observations in the data set under the assumption of a cross-sectional study. The rest of the variables in this estimator are multinomial random variables with parameters $n$ and $\hat{p}_{i}=\frac{i}{n} \forall i$ where $i$ takes on the value of each cell in the $2 \times 2$ table. Once again this estimator is frequently used with a log transformation to closer approximate a symmetric distribution yielding, $\ln (R O R)=\ln \left(n_{i j}\right)+\ln \left(n_{. .}-n_{. j}-n_{i .}+n_{i j}\right)-\ln \left(n_{. j}-n_{i j}\right)-\ln \left(n_{i .}-n_{i j}\right)$. Taking the expectation we get $E(\ln (R O R)) \approx \ln \left(E\left(n_{i j}\right)\right)+\ln \left(E\left(n_{. .}-n_{. j}-n_{i .}+n_{i j}\right)\right)-\ln \left(E\left(n_{. j}-n_{i j}\right)\right)-\ln \left(E\left(n_{i .}-n_{i j}\right)\right)$

$$
E(\ln (R O R)) \approx \ln \left(n_{i j}\right)+\ln \left(n_{. .}-n_{. j}-n_{i .}+n_{i j}\right)-\ln \left(n_{. j}-n_{i j}\right)-\ln \left(n_{i .}-n_{i j}\right)
$$

Again, our estimator is approximately unbiased using a log transformation. However, a multivariate Taylor series (1.4.1) can give us an idea of the bias associated with this estimator. Once again, 
Let $A=n_{i j}, B=n_{i .}-n_{i j}, C=n_{. j}-n_{i j}$ and $D=n_{. .}-n_{. j}-n_{i .}+n_{i j}$ and $n=n_{\text {.. }}$

Then

$$
R O R=\frac{\frac{n_{i j}}{n_{i .}} / \frac{n_{i .}-n_{i j}}{n_{i .}}}{\frac{n_{. j}-n_{i j}}{n_{. .}-n_{. j}} / \frac{n_{.}-n_{. j}-n_{i .}+n_{i j}}{n_{. .}-n_{. j}}}=\frac{n_{i j}\left(n_{.}-n_{. j}-n_{i .}+n_{i j}\right)}{\left(n_{. j}-n_{i j}\right)\left(n_{i .}-n_{i j}\right)}=\frac{A D}{B C}
$$

where

$$
A, B, C, D \sim \text { multinomia } l\left(n, p_{i}\right) \quad i=a, b, c, d \text { and } p_{i}=\frac{i}{n} \forall i
$$

Let $\Theta=(A, B, C, D)^{T}$ where $A, B, C, D$ represent the so-called 'true' values of the cells in the $2 \times 2$ table, which are estimated by $\theta=(a, b, c, d)^{T}$.

Taking the expectation of a second order multivariate Taylor series expansion about $\Theta=\theta$ yields:

$E(f(\Theta)) \approx f(\theta)+\frac{1}{2} E\left[(\Theta-\theta) \mathrm{H}(\theta)(\Theta-\theta)^{T}\right]$ since $E(\Theta-\theta)=0$. Substituting the partial derivatives make the Hessian matrix:

$$
\mathrm{H}(\boldsymbol{\theta})=\left(\begin{array}{cccc}
0 & \frac{-d}{b^{2} c} & \frac{-d}{b c^{2}} & \frac{1}{b c} \\
\frac{-d}{b^{2} c} & \frac{a d}{2 b^{3} c} & \frac{a d}{b^{2} c^{2}} & \frac{-a}{b^{2} c} \\
\frac{-d}{b c^{2}} & \frac{a d}{b^{2} c^{2}} & \frac{a d}{2 b c^{3}} & \frac{-a}{b c^{2}} \\
\frac{1}{b c} & \frac{-a}{b^{2} c} & \frac{-a}{b c^{2}} & 0
\end{array}\right)
$$


Finally, the second term, the bias, reduces to $\frac{a d}{4 c b}\left(\frac{\operatorname{Var}(b)}{b^{2}}+\frac{\operatorname{Var}(c)}{c^{2}}\right)$ which means the $E(R O R) \approx \frac{a d}{c b}\left(1+\frac{1}{4}\left(\frac{\operatorname{Var}(b)}{b^{2}}+\frac{\operatorname{Var}(c)}{c^{2}}\right)\right)$. Finally, substituting the formulas for the variance terms into the expression of the bias yields

$$
\begin{gathered}
\frac{n a d}{4 c b}\left(\frac{p_{B}\left(1-p_{B}\right)}{b^{2}}+\frac{p_{C}\left(1-p_{C}\right)}{c^{2}}\right)=\frac{a d}{4 c b n}\left(\frac{n-b}{b}+\frac{n-c}{c}\right) \\
=\frac{a d}{4 c b^{2}}+\frac{a d}{4 c^{2} b}-\frac{a d}{2 c b n}=\frac{a d(b+c)}{4 b^{2} c^{2}}-\frac{a d}{2 c b n}
\end{gathered}
$$

Thus the size of the bias is determined by the difference in $\frac{a d(b+c)}{4 b^{2} c^{2}}$ and $\frac{a d}{2 c b n}$.

Once again it is appropriate to consider the bias's behaviour as $n \rightarrow \infty$. Clearly, the second term will go to zero as there is an $n$ in the denominator. For the first term we have a similar issue as with the PRR. Therefore, to assess the asymptotic behaviour of the ROR we will consider all possibilities of convergence.

For the behaviour of $b$ we have $\lim _{b \rightarrow \infty} \frac{a d(b+c)}{4 b^{2} c^{2}}=\lim _{b \rightarrow \infty} \frac{\frac{a d b}{b^{2}}+\frac{a d c}{b^{2}}}{4 c^{2}}=\frac{0}{4 c^{2}}=0$ and the same argument will apply to $c$ as well. For $a$ and $d$ however, it is not so straightforward. We can rewrite the expression as $a d\left(\frac{1}{4 b c^{2}}+\frac{1}{4 b^{2} c}\right)$ which implies that $\lim _{a \rightarrow \infty} a d\left(\frac{1}{4 b c^{2}}+\frac{1}{4 b^{2} c}\right)=\infty$ and the same for $d$. However, in pharmacovigilance this type 
of problem rarely occurs for $a$. As $a$ gets large, generally all other values are getting large as well. However $d$ is a bit more suspicious since it represents the total of all observed interactions in the complement set of drugs and ADRs. Therefore it is important to assess the size of $d$ when considering the bias. A loose guide for the relative size of $d$ is to consider a scenario where $a \approx b \approx c$. Under this condition, we can see that the overall exponent in the numerator is four and in the denominator it is three.

Therefore if $d \geq c^{2}$ the exponents will be of the same order and the bias will be noticeable. Practically, this approximation is relatively close to reality with the exception of $a$. It is generally the smallest term and is dominated by $b$ and $c$. However this adjustment gives us even more leeway as it will shrink the numerator and require a larger value for $d$ to make the bias noticeable. Finally, resubstituting our previous notation into the expression for the bias yields,

$$
\frac{n_{i j}\left(n-n_{. j}-n_{i .}+n_{i j}\right)}{2\left(n_{i .}-n_{i j}\right)\left(n_{. j}-n_{i j}\right)}\left(\frac{1}{2\left(n_{i .}-n_{i j}\right)}+\frac{1}{2\left(n_{. j}-n_{i j}\right)}-\frac{1}{n_{. .}}\right) .
$$

Similarly to the PRR, the variance calculation for the ROR is done logarithmically.

Let $A=n_{i j}, B=n_{i .}-n_{i j}, C=n_{. j}-n_{i j}$ and $D=n_{. .}-n_{. j}-n_{i .}+n_{i j}$

Then

$$
R O R=\frac{\frac{n_{i j}}{n_{i .}} / \frac{n_{i .}-n_{i j}}{n_{i .}}}{\frac{n_{. j}-n_{i j}}{n_{. .}-n_{. j}} / \frac{n_{. .}-n_{. j}-n_{i .}+n_{i j}}{n_{. .}-n_{. j}}}=\frac{n_{i j}\left(n-n_{. j}-n_{i .}+n_{i j}\right)}{\left(n_{. j}-n_{i j}\right)\left(n_{i .}-n_{i j}\right)}=\frac{A D}{B C}
$$


Let

$$
A, B, C, D \sim \operatorname{multinomial}\left(n, p_{i}\right) \quad i=a, b, c, d \quad p_{i}=\frac{i}{n} \forall i
$$

Since $R O R=\frac{A D}{B C} \frac{n^{-2}}{n^{-2}}=\frac{p_{A} p_{D}}{p_{B} p_{C}}$

$$
g\left(p_{A}, p_{B}, p_{C}, p_{D}\right)=\ln (R O R)=\ln \left(p_{A}\right)+\ln \left(p_{D}\right)-\ln \left(p_{B}\right)-\ln \left(p_{C}\right)
$$

Then define, $\boldsymbol{\varphi}=\left[\left.\frac{d g}{d p_{A}}\right|_{A=a},\left.\frac{d g}{d p_{B}}\right|_{B=b},\left.\frac{d g}{d p_{C}}\right|_{C=c},\left.\frac{d g}{d p_{D}}\right|_{D=d}\right]^{T}=\left[\frac{1}{p_{a}}, \frac{-1}{p_{b}}, \frac{-1}{p_{c}}, \frac{1}{p_{d}}\right]^{T}$

By the Multivariate Delta Method (1.4.3), the asymptotic variance of $\sqrt{n}[g(\hat{\mathbf{p}})-g(\mathbf{p})]$ is $\varphi^{T} \Sigma \varphi$ where $\Sigma$ is the covariance matrix of $\hat{\mathbf{p}}$.

To solve the covariance matrix of $\hat{\mathrm{p}}$, for each $i$,

$$
\begin{aligned}
& E\left(\hat{p}_{i}\right)=E\left(\frac{i}{n}\right)=\frac{1}{n} E(i)=\frac{n p_{i}}{n}=p_{i} \\
& \operatorname{Var}\left(\hat{p}_{i}\right)=\operatorname{Var}\left(\frac{i}{n}\right)=\frac{1}{n^{2}} \operatorname{Var}(i)=\frac{n p_{i}\left(1-p_{i}\right)}{n^{2}}=\frac{p_{i}\left(1-p_{i}\right)}{n}
\end{aligned}
$$

To solve $\operatorname{Cov}\left(\hat{p}_{j}, \hat{p}_{k}\right)$ where $j$ and $k$ represent two different outcomes,

define $Y_{i j}=\left\{\begin{array}{rr}1 & \text { if the ith observation has outcome } j \\ 0 & \text { otherwise }\end{array}\right.$

This implies that $\hat{p}_{j}=\frac{\sum_{i} Y_{i j}}{n}$ and that $Y_{i j} Y_{i k}=0, \forall j \neq k$.

$\operatorname{Cov}\left(Y_{i j}, Y_{i k}\right)=0-E\left(Y_{i j}\right) E\left(Y_{i k}\right)=-\hat{p}_{j} \hat{p}_{k}$ since $Y_{i j} \sim \operatorname{Bernoulli}\left(p_{j}\right)$. 
The covariance matrix

$$
\begin{aligned}
& \operatorname{Cov}(\mathbf{Y})=\left[\operatorname{Cov}\left(Y_{j}, Y_{k}\right)\right]_{n x n}=\left[\begin{array}{cccc}
p_{1}\left(1-p_{1}\right) & -p_{1} p_{2} & \ldots & -p_{1} p_{n} \\
-p_{2} p_{1} & p_{2}\left(1-p_{2}\right) & & \ldots \\
\ldots & & \ldots & \\
-p_{n} p_{1} & \ldots & & p_{n}\left(1-p_{n}\right)
\end{array}\right] \\
& =\operatorname{diag}(\mathbf{p})-\mathbf{p p}^{T} \\
& \text { Thus, } \sum=\operatorname{Cov}(\mathbf{P})=\operatorname{Cov}\left(\frac{\sum_{i} \mathbf{Y}_{i}}{n}\right)=\frac{1}{n^{2}} \operatorname{Cov}\left(\sum_{i} \mathbf{Y}_{i}\right)=\frac{1}{n^{2}} \sum_{i} \operatorname{Cov}\left(\mathbf{Y}_{i}\right) \\
& =\frac{1}{n^{2}} \sum_{i}\left(\operatorname{diag}(\mathbf{p})-\mathbf{p} \mathbf{p}^{T}\right)=\frac{\operatorname{diag}(\mathbf{p})-\mathbf{p} \mathbf{p}^{T}}{n}
\end{aligned}
$$

Finally,

$$
\sqrt{n}[g(\hat{\mathbf{p}})-g(\mathbf{p})] \stackrel{d}{\longrightarrow} N\left(0, \varphi^{T}\left[\operatorname{diag}(\mathbf{p})-\mathbf{p p}^{T}\right] \varphi\right)
$$

Therefore, the asymptotic variance of $\sqrt{n}[g(\hat{\mathbf{p}})-g(\mathbf{p})]$ is

$$
\begin{gathered}
\boldsymbol{\varphi}^{T}\left[\operatorname{diag}(\mathbf{p})-\mathbf{p p}^{T}\right] \varphi=\varphi^{T} \operatorname{diag}(\mathbf{p}) \varphi-\varphi^{T} \mathbf{p p}^{T} \boldsymbol{\varphi} \\
=\varphi^{T} \operatorname{diag}(\mathbf{p}) \varphi=\sum p_{i} \phi_{i}^{2}=p_{a}\left(\frac{1}{p_{a}}\right)^{2}+p_{b}\left(\frac{-1}{p_{b}}\right)^{2}+p_{c}\left(\frac{-1}{p_{c}}\right)^{2}+p_{d}\left(\frac{1}{p_{d}}\right)^{2} \\
=\frac{1}{p_{a}}+\frac{1}{p_{b}}+\frac{1}{p_{c}}+\frac{1}{p_{d}}
\end{gathered}
$$

because $\mathbf{p}^{T} \boldsymbol{\varphi}=0$.

Finally, $V(\ln (R O R)) \approx \frac{\varphi^{T} \operatorname{diag}(\mathbf{p}) \varphi}{n}$ and 


$$
V(\ln (R O R)) \approx \frac{1}{n_{i j}}+\frac{1}{n_{. j}-n_{i j}}+\frac{1}{n_{i .}-n_{i j}}+\frac{1}{n_{. .}-n_{. j}-n_{i .}+n_{i j}}
$$

The range of the ROR, zero to infinity, warrants some discussion with respect to it interpretability. The larger the ROR, the stronger the association between the two variables involved. However, for values of the ROR less than one, we see an opposite effect. An ROR of less than one implies that there is a greater probability that a signal exists with respect to something other than the drug in question, and that the magnitude of the association is lessened. Unfortunately, these scales are not symmetric and it is difficult to interpret values between zero and one.

Yule's Q is frequently used to address this issue. Yule's Q employs a one-to-one transformation of the ROR to adjust its range to include both negative and positive

values, $Q=\frac{R O R-1}{R O R+1}=\frac{\frac{n_{i j}\left(n . .-n_{. j}-n_{i .}+n_{i j}\right)-\left(n_{. j}-n_{i j}\right)\left(n_{i .}-n_{i j}\right)}{\left(n_{. j}-n_{i j}\right)\left(n_{i .}-n_{i j}\right)}}{\frac{n_{i j}\left(n_{. .}-n_{. j}-n_{i .}+n_{i j}\right)+\left(n_{. j}-n_{i j}\right)\left(n_{i .}-n_{i j}\right)}{\left(n_{. j}-n_{i j}\right)\left(n_{i .}-n_{i j}\right)}}$, so

$$
Q=\frac{n_{i j}\left(n-n_{. j}-n_{i .}+n_{i j}\right)-\left(n_{. j}-n_{i j}\right)\left(n_{i .}-n_{i j}\right)}{n_{i j}\left(n_{. .}-n_{. j}-n_{i .}+n_{i j}\right)+\left(n_{. j}-n_{i j}\right)\left(n_{i .}-n_{i j}\right)}
$$

This symmetric range is much easier to interpret, and since it essentially implies the same sort of inference with negative and positive values, it has been favourably applied in pharmacovigilance. Yule's Q has zero expectation under the null hypothesis with variance 


$$
V(Q) \approx\left(\frac{1}{2}\left(1-Q^{2}\right)\right)^{2}\left(\frac{1}{n_{i j}}+\frac{1}{n_{. j}-n_{i j}}+\frac{1}{n_{i .}-n_{i j}}+\frac{1}{n_{. .}-n_{. j}-n_{i .}+n_{i j}}\right)
$$

Since the bias of the $R O R$ went to zero as $n \rightarrow \infty$ and we already have information on $V(\ln (R O R))$ these properties can be derived using the delta method.

Let $A=n_{i j}, B=n_{i .}-n_{i j}, C=n_{. j}-n_{i j}$ and $D=n_{. .}-n_{. j}-n_{i .}+n_{i j}$

Let $R=\ln (R O R)=\ln \left(\frac{A D}{B C}\right)$ then $\operatorname{Var}(R)=\frac{1}{a}+\frac{1}{b}+\frac{1}{c}+\frac{1}{d}$ and $Q=\frac{e^{R}-1}{e^{R}+1}$.

By the delta method (1.4.3),

$$
\begin{aligned}
& E(Q)=Q \text { and } \\
& \begin{aligned}
\operatorname{Var}(Q) \approx\left(\frac{1}{a}+\frac{1}{b}+\frac{1}{c}+\frac{1}{d}\right)\left(\frac{2 e^{R}}{\left(e^{R}+1\right)^{2}}\right)^{2}=\left(\frac{1}{a}+\frac{1}{b}+\frac{1}{c}+\frac{1}{d}\right)\left(\frac{4 R O R}{2(R O R+1)^{2}}\right)^{2} \\
=\left(\frac{1}{a}+\frac{1}{b}+\frac{1}{c}+\frac{1}{d}\right)\left(\frac{4 R O R+R O R^{2}-R O R^{2}+1-1}{2(R O R+1)^{2}}\right)^{2} \\
=\left(\frac{1}{a}+\frac{1}{b}+\frac{1}{c}+\frac{1}{d}\right)\left(\frac{(R O R+1)^{2}-(R O R-1)^{2}}{2(R O R+1)^{2}}\right)^{2} \\
=\left(\frac{1}{a}+\frac{1}{b}+\frac{1}{c}+\frac{1}{d}\right)\left(\frac{1}{2}-\left(\frac{R O R-1}{R O R+1}\right)^{2}\right)^{2}=\left(\frac{1}{a}+\frac{1}{b}+\frac{1}{c}+\frac{1}{d}\right)\left(\frac{1}{2}\left(1-Q^{2}\right)\right)^{2}
\end{aligned}
\end{aligned}
$$


The availability of confidence intervals for both of these measures is an asset in signal detection. Specifically, the lower bound of the confidence interval should exceed the expectation of the measure in question in order to provide statistically significant evidence of a signal. Van Puijenbroek et al. (van Puijenbroek et al., 2002) use a cutoff of 1 for the lower bound of the ROR, and Roux et al. (Roux et al., 2005) use a cutoff of 0 for the lower bound of Yule's Q. Note that given the small number of reports, these confidence intervals can have some issues since the distributions can become skewed, and as such a normally distributed confidence interval will exhibit some problems. Thus a technique used in some simulations is to not consider drug/ADR pairs below a certain number of reports.

As previously stated, one characteristic of the PRR that Rothman et al. (Rothman et al., 2004) criticize is the relationship with risk. They imply that the ROR is a much better estimator due to its consistency with respect to the risk ratio and its ability to reduce some of the biases that plague the PRR. The first bias they mention relates to the use of a control group used to gain information about the background incidence level of ADRs. If this group includes adverse reactions associated with the drug in question, the background incidence rate for that health outcome will be overestimated. The ROR is capable of bringing these interactions to light, and, as such, offers a method for reducing this bias. The second bias Rothman refers to relates to the fact that the PRR can be biased by an extreme observation at another place in the row or column, causing the denominator to inflate, thus biasing the estimator downward. Due to the algebraic 
cancellations that occur in the ROR, these potentially skewing large values are not of issue.

In response to this concern, Waller et al. (Waller et al., 2004) assert that these so called advantages are of minor importance in the practical sense. First of all, the risk ratio calculations appear biased under the condition that $n_{i j}$ and $n_{. j}-n_{i j}$ (the two numerators in the calculation of the PRR) are large compared to their respective denominators. This is not the case in pharmacovigilance as we are generally dealing with rare events. In fact, the opposite is true: if these numerators are small with respect to their corresponding denominators, the PRR and ROR will give similar results with respect to the risk ratio. Finally, Waller et al deal with the issue of inflated denominators by stating that there is no mathematical issue with simply removing the biasing cell from these calculations.

Finally, we note that under the assumption that the success probabilities are small, the odds ratio approximates the relative risk. The same can be said in this application given the discussion of the previous paragraph. The argument made by Waller et al. demonstrates that when the two numerators are low, the estimated risk ratios are similar; this can be extended to assert that under the preceding condition, the ROR will approximate the PRR. Thus, these two estimators seem to be investigating the same thing; however, they will perform differently under different conditions. 


\subsection{Sequential tests}

Sequential tests examine the effect of adding newly observed sample points to an existing sequence and monitoring their effect on signal detection. The first sequential test considered here is known as the cumulative sum technique. Although this method has not been shown to perform favourably, we will briefly consider its properties. The concept underlying the cumulative sum technique is simple; if addition of new information to the sequence causes the deviations about the mean to increase or decrease, then an underlying process is presumed to be affecting these new observations.

Essentially, upper and lower boundaries are placed around a reference statistic, usually the mean, and the system detects a signal if these boundaries are transgressed. This basic algorithm leads into the sequential probability ratio test, which has performed favourably in pharmacovigilance.

The sequential probability ratio test (SPRT) is similar in nature; however the boundaries and reference statistic are different. This technique, originally published by Wald (Wald, 1945), looks at upper and lower bounds made up of a ratio of Type 1 and Type 2 errors. Also, the reference statistic is a likelihood ratio statistic.

Let $g_{i}$ represent the prior probability that $H_{i} i=0,1$ is true. 
Then by Bayes rule, $g_{i m}=\frac{g_{i} p_{i m}\left(x_{1}, \ldots x_{n}\right)}{g_{0} p_{0 m}\left(x_{1}, \ldots x_{n}\right)+g_{1} p_{1 m}\left(x_{1}, \ldots x_{n}\right)} i=0,1$ and $p_{i m}\left(x_{1}, \ldots x_{n}\right)$ is the pdf of the null or alterative space. He denotes these joint distributions as $p_{i m} i=0,1$ for simplicity's sake.

Let $d_{i} \in[0.5,1], i=0,1$, such that

$$
P\left(H_{0} \text { is accepted } \mid H_{0} \text { true }\right) \geq d_{0} \quad P\left(H_{1} \text { is accepted } \mid H_{1} \text { true }\right) \geq d_{1}
$$

This implies that we should accept $H_{0}$ if $g_{0 m} \geq d_{0}$ and accept $H_{1}$ if $g_{1 m} \geq d_{1}$. The $d_{i}$ 's have the numerical restriction to avoid a situation where the hypothesis with the smaller posterior will be accepted.

Thus, we reject $H_{i}$ if $g_{i m}=\frac{g_{i} p_{i m}}{g_{0} p_{0 m}+g_{1} p_{1 m}} \geq d_{i}$. Note that this defines three regions corresponding to the decisions above. The null and alternative space is previously defined and Wald denotes these as $R_{m}^{0}$ and $R_{m}^{1}$ respectively. The other region, denoted by $R_{m}$ represents the situation where both of the previous inequalities are not satisfied or, $g_{1 m}<d_{1}$ and $g_{0 m}<d_{0}$. Under this circumstance the decision is to keep sampling and updating the posterior probabilities until a clear decision is reached. An important implied assumption of these regions is that they are mutually exclusive, or their intersection must be zero. 
The next step in implementation of this test is to note that the information most accessible is the ratio of the joint pdf's, or likelihood ratio, corresponding to the probability of being in null or alternative space. With this goal in mind we can make the decision to accept $H_{i}, i=0,1$ if:

$$
g_{i m}=\frac{g_{i} p_{i m}}{g_{0} p_{0 m}+g_{1} p_{1 m}} \geq d_{i} \Rightarrow g_{i} p_{i m} \geq d_{i}\left(g_{0} p_{0 m}+g_{1} p_{1 m}\right) \text { which leads to }
$$

$$
\left\{\begin{array}{l}
\frac{p_{1 m}}{p_{0 m}} \geq \frac{g_{0}}{g_{1}} \frac{d_{1}}{1-d_{1}}=A \Rightarrow \text { Accept } H_{1} \\
\frac{p_{1 m}}{p_{0 m}} \leq \frac{g_{0}}{g_{1}} \frac{1-d_{0}}{d_{0}}=B \Rightarrow \text { Accept } H_{0} \\
\text { Otherwise, continue to sample }
\end{array}\right.
$$

Since we now have expressions that lead to the required decisions without any knowledge of the priors, we have developed a way to make decisions simply by looking at the null and alternative pdfs. Also Wald puts the restriction that if $p_{0 m}=p_{1 m}=0$ then the likelihood ratio is defined as 1 .

The values of $\mathrm{A}$ and $\mathrm{B}$ are chosen such that $0<B<A$, and the test has appropriately chosen values for the type I and type II errors. Wald went on to derive appropriate thresholds for this test. If we define $Q_{i}$ to be the sum of all points leading to the acceptance of $H_{i}$, and $P_{j}(X)$ to be the probability of $X$ given that $H_{j}$ is true, then we can create the following inequalities: 


$$
P_{1}\left(Q_{1}\right) \geq A P_{0}\left(Q_{1}\right) \quad P_{1}\left(Q_{0}\right) \leq B P_{0}\left(Q_{0}\right)
$$

Furthermore, note that by definition, $P_{0}\left(Q_{1}\right)=\alpha$ and $P_{1}\left(Q_{0}\right)=\beta$, where $\alpha$ is the probability of rejecting $H_{0}$ given that it is true (Type 1 error) and $\beta$ is the probability of rejecting $H_{1}$ given that it is true (type 2 error). Since the $Q_{i}$ 's are disjoint, it follows that $P_{1}\left(Q_{1}\right)=1-\beta$ and $P_{0}\left(Q_{o}\right)=1-\alpha$. Plugging these back into the inequalities and solving for $\mathrm{A}$ and $\mathrm{B}$ gives the following boundaries for our test: $A=\frac{1-\beta}{\alpha}$ and $B=\frac{\beta}{1-\alpha}$.

Keep in mind that the values of $\alpha$ and $\beta$ are chosen by the experimenter, and should reflect practical values given their definitions. In pharmacovigilance, Evans (Evans, 2003) proposed the application of this technique as a method of signal detection. Roux et al (Roux et al., 2005) developed a specific form of the test assuming that the pdfs are Poisson distributed, and they assert that a signal can be defined by a relative risk twice that of a drug/ADR pair with no association. They also chose a value of 0.05 for both types of error.

Let $A=n_{i j}, B=n_{i .}-n_{i j}, C=n_{. j}-n_{i j}$ and $D=n_{. .}-n_{. j}-n_{i .}+n_{i j}$

Let $R R=\frac{\frac{a}{a+b}}{\frac{c}{c+d}}$ and $E=\frac{(a+b)(c+d)}{N}$ where $N=a+b+c+d=n$.. Solving these equations we get $R R=\frac{a(c+d)^{2}}{c E N}$. According to Roux et al's description of the rejection 
region, $R R_{0}=\frac{a(c+d)^{2}}{c E N}$ and $R R_{1}=\frac{2 a(c+d)^{2}}{c E N}$ which implies that $E_{0}=\frac{a(c+d)^{2}}{R R c N}$ and $E_{1}=\frac{2 a(c+d)^{2}}{R R c N}$. Since the pdfs associated with the null and alternative spaces are both

Poisson with $E_{i} i=0,1$ as the parameter, we get $\frac{p_{1 m}}{p_{0 m}}=\frac{e^{-E_{1}} E_{1}^{a} / a !}{e^{-E_{0}} E_{0}^{a} / a !}=e^{E_{0}-E_{1}}\left(\frac{E_{1}}{E_{0}}\right)^{a}$.

Taking the natural logarithm yields

$E_{0}-E_{1}+a \ln \left(\frac{E_{1}}{E_{0}}\right)=\frac{a(c+d)^{2}}{R R c N}-\frac{2 a(c+d)^{2}}{R R c N}+a \ln 2=-\frac{a(c+d)^{2}}{R R c N}+a \ln (2)=-E_{0}+a \ln (2)$

Thus,

$$
\ln (2) \cdot a-E \geq \ln \left(\frac{1-\beta}{\alpha}\right)
$$

implies that a signal has been identified.

This test is attractive in that its purpose is to assess the point at which the probability of incorrectly identifying a non-signal is minimized. This leads to a situation where false negatives can occur, since the boundaries set by Roux et al do not include an upper bound. In other words, this test will incorrectly identify an interaction as a signal rather than to leave out a signal due to the over-correctness of the model. How one views this fact with respect to pharmacovigilance is subjective and the trade off between sensitivity and specificity with respect to hypothesis testing in general is a discussion frequently had in applied areas of statistics. It is difficult to comment on what would be more ideal in this application, either to over flag signals and expend resources 
researching the validity of these hypotheses, or to minimize the flagging of false signals and potentially miss some real ADR/drug pairs. This debate is not one that is covered in the scope of this thesis and this question will be left for another time. 


\section{Chapter 3}

\section{Theoretical Discussion of Signal Detection Methods - Bayesian Approach}

A reoccurring criterion for signal detection is the reporting rate, or the ratio of the observed to the expected count of adverse reactions. The random variable, $R R=n_{i j} / E_{i j}$, is subject to sampling variability. Thus, for small sample sizes, estimates of the reporting rate will be highly variable. In order to deal with this problem, Bayesian methods have been proposed to enhance the accuracy with which the reporting rate is estimated through the use of prior distributions on these quantities, and observing the effect that more and more information has on the posterior distributions.

There are two major methods in this category, one used by the World Health Organization, known as the Bayesian Confidence Propogation Neural Network (BCPNN) or simply the WHO method (Bate et al., 1998; Orre et al., 2000; Bate et al., 2002; Bate and Edwards, 2006; Bate, 2007), and the empirical Bayesian Screening method (EBS) (Dumouchel, 1999; Szarfman et al., 2002; Gould, 2003). 


\subsection{Bayesian Confidence Propogation Neural Network (BCPNN)}

This method considers the probability of an adverse medical condition, $A_{j}$, to be represented by the prior probability $P\left(A_{j}\right)$. After addition of information about a specific drug, we get the posterior probability of this adverse condition, $P\left(A_{j} \mid D_{i}\right)$, given drug $D_{i}$. If $P\left(A_{j} \mid D_{i}\right)>P\left(A_{j}\right)$, this implies that the additional information added by drug $D_{i}$ has increased this probability and a signal is perceived. Baye's rule demonstrates that these probabilities are related by the multiplicative factor:

$$
P\left(A_{j} \mid D_{i}\right)=\frac{P\left(A_{j}, D_{i}\right)}{P\left(D_{i}\right)}=P\left(A_{j}\right) \frac{P\left(A_{j}, D_{i}\right)}{P\left(A_{j}\right) P\left(D_{i}\right)},
$$

where $P\left(A_{j}, D_{i}\right)$ is the joint probability of simultaneously observing $A_{j}$ and $D_{i}$, and $P\left(D_{i}\right)$ is the marginal probability of observing drug $D_{i}$. Thus for values of

$\frac{P\left(A_{j}, D_{i}\right)}{P\left(A_{j}\right) P\left(D_{i}\right)}>1$, the previous inequality holds, and a signal is detected. Note that if $A_{j}$ and $D_{i}$ are independent, the posterior and prior will be equal, implying that under independence, the addition of information about the drug $D_{i}$ has no bearing on the prior probability, and no signal detected. In information theory, this ratio is discussed under the name "Information Component (IC)", or the strength of association between two variables, and is defined with respect to logarithms: 


$$
I C_{i j}=\log _{2}\left(\frac{P\left(A_{j}, D_{i}\right)}{P\left(A_{j}\right) P\left(D_{i}\right)}\right)
$$

Under the logarithmic form signals are detected for values of $I C_{i j}>0$.

Since the IC is an estimate of the 'true' factor relating these probabilities, the initial estimates may be extremely variable, especially under the small sample size condition. To deal with this variability beta distributions are applied to the prior components of the IC specifically, $P\left(A_{j}\right) \sim \operatorname{Beta}\left(\alpha_{1}, \alpha_{0}\right)$ and $P\left(D_{i}\right) \sim \operatorname{Beta}\left(\beta_{1}, \beta_{0}\right)$. These probabilities represent the probability that the event $A_{j}$ or $D_{i}$ take place, or more generally, they define the success probability in a Bernoulli trial. For the joint distribution, $P\left(A_{j}, D_{i}\right)$, there are four possible outcomes, $P\left(A_{j}=u, D_{i}=v\right) \forall u, v=0,1$, and thus a three-dimensional Dirichlet distribution with parameters $\gamma_{u v}$ is used. Practically, the only event that is of interest for our joint distribution is $\left\{A_{j}=1, D_{i}=1\right\}$. Thus we must determine the marginal distribution of this event. First note that the Dirichlet distribution is generated as follows:

Let $X_{i} \sim \Gamma\left(\alpha_{i}, \theta\right)$ then $\left(Y_{1}, \ldots, Y_{n}\right)=\left(\frac{X_{1}}{\sum_{j=1}^{n+1} X_{j}}, \ldots, \frac{X_{n}}{\sum_{j=1}^{n+1} X_{j}}\right) \sim$ Dirichlet $_{n}\left(\alpha_{1}, \ldots, \alpha_{n+1}\right)$

(Balakrishnan and Nevzorov, 2003) 
Thus the marginal for the $k^{\text {th }}$ element is equivalent in distribution to

$Y_{k} \stackrel{d}{=} \frac{X_{k}}{\sum_{j=1}^{n+1} X_{j}}=\frac{X_{k}}{X_{k}+\left(\sum_{j=1}^{n+1} X_{j}-X_{k}\right)}$. By the properties of the Gamma distribution,

$\sum_{j=1}^{n+1} X_{j} \sim \operatorname{Gamma}\left(\sum_{i} \alpha_{i}, \theta\right)$ which implies that $\sum_{j=1}^{n+1} X_{j}-X_{k} \sim \operatorname{Gamma}\left(\sum_{i} \alpha_{i}-\alpha_{k}, \theta\right)$

Thus the marginal $Y_{k}$ is represented by the well known transformation used to generate beta random variables, and the resulting parameters are $\alpha_{k}$ and $\sum_{i} \alpha_{i}-\alpha_{k}$. Thus we can express the marginal of $\left\{A_{j}=1, D_{i}=1\right\}$ by $P\left(A_{j}=1, D_{i}=1\right) \sim \operatorname{Beta}\left(\delta_{1}, \delta_{0}\right)$ where $\delta_{1}=\gamma_{11}, \delta_{0}=\gamma-\gamma_{11}$ where $\gamma=\sum_{u, v} \gamma_{u v} u, v=0,1$

Note that Gould (Gould, 2003) defines $\delta_{0}$ and $\delta_{1}$ as follows:

$\delta_{1}=1, \delta_{0}=\frac{1-E\left(A_{j}\right) E\left(D_{i}\right)}{E\left(A_{j}\right) E\left(D_{i}\right)}$ where $E\left(A_{j}\right)=\frac{\alpha_{1}}{\alpha_{1}+\alpha_{0}} \quad E\left(D_{i}\right)=\frac{\beta_{1}}{\beta_{1}+\beta_{0}}$. The posteriors are obtained by conditioning on the marginal counts as follows. Using the notation (1.3.2), the required posterior distributions are $P\left(A_{j} \mid a+c, b+d\right), P\left(D_{i} \mid a+b, c+d\right)$ and $P\left(A_{j}, D_{i} \mid a, b, c, d\right)$. To start we will consider the first probability distribution, $P\left(A_{j} \mid a+c, b+d\right)$. 
Let $A=n_{i j}, B=n_{i .}-n_{i j}, C=n_{. j}-n_{i j}, D=n_{. .}-n_{. j}-n_{i .}+n_{i j}$ and $N=A+B+C+D$

Then $A+C$ represents the number of times ADR $A_{j}$ was observed in the table and $B+D$ represents the total number of times $A_{j}$ was not the observed ADR, or the total for the complement set. Each of these can be represented as a binomial random variable representing the number of so-called successes and failures for the variable $A_{j}$, or $A+C \sim \operatorname{Binomial}\left(N, \frac{a+c}{N}\right)$ and $B+D \sim \operatorname{Binomial}\left(N, \frac{b+d}{N}\right) . \quad$ Thus the joint distribution conditioned on the random variable $A_{j}$ is also binomial and has the probability mass function $p\left(A+C, B+D \mid A_{j}=a_{j}\right)=\left(\begin{array}{c}N \\ a+c\end{array}\right) a_{j}^{a+c}\left(1-a_{j}\right)^{b+d}$.

Finally we have,

$$
\begin{aligned}
P\left(A_{j} \mid a+c, b+d\right) & =\frac{p\left(A+C, B+D \mid A_{j}=a_{j}\right) p\left(A_{j}=a_{j}\right)}{\int p\left(A+C, B+D \mid A_{j}=a_{j}\right) p\left(A_{j}=a_{j}\right) d a_{j}} \\
& =\frac{\left(\begin{array}{c}
N \\
a+c
\end{array}\right) a_{j}^{a+c}\left(1-a_{j}\right)^{b+d} \frac{\Gamma\left(\alpha_{1}+\alpha_{0}\right)}{\Gamma\left(\alpha_{1}\right) \Gamma\left(\alpha_{0}\right)} a_{j}^{\alpha_{1}-1}\left(1-a_{j}\right)^{\alpha_{0}-1}}{\int\left(\begin{array}{c}
N \\
a+c
\end{array}\right) a_{j}^{a+c}\left(1-a_{j}\right)^{b+d} \frac{\Gamma\left(\alpha_{1}+\alpha_{0}\right)}{\Gamma\left(\alpha_{1}\right) \Gamma\left(\alpha_{0}\right)} a_{j}^{\alpha_{1}-1}\left(1-a_{j}\right)^{\alpha_{0}-1} d a_{j}} \\
& =\frac{a_{j}^{a+c+\alpha_{1}-1}\left(1-a_{j}\right)^{b+d+\alpha_{0}-1}}{\left[\frac{\Gamma(N+1)}{\Gamma(a+c+1) \Gamma(c+d+1)} \frac{\Gamma\left(\alpha_{1}+\alpha_{0}\right)}{\Gamma\left(\alpha_{1}\right) \Gamma\left(\alpha_{0}\right)}\right]^{-1}}
\end{aligned}
$$




$$
=\frac{\Gamma\left(N+\alpha_{1}+\alpha_{0}\right)}{\Gamma\left(a+c+\alpha_{1}\right) \Gamma\left(c+d+\alpha_{0}\right)} a_{j}^{a+c+\alpha_{1}-1}\left(1-a_{j}\right)^{b+d+\alpha_{0}-1}
$$

Thus $P\left(A_{j} \mid a+c, b+d\right)=P\left(A_{j} \mid n_{. j}, n_{. .}-n_{. j}\right) \sim \operatorname{Beta}\left(\alpha_{1}+n_{. j}, \alpha_{0}+n_{. .}-n_{. j}\right)$. The same logic can be used to produce the posterior distribution $P\left(D_{i} \mid n_{. j}, n_{. .}-n_{. j}\right) \sim \operatorname{Beta}\left(\beta_{1}+n_{. j}, \beta_{0}+n_{. .}-n_{. j}\right)$.

Solving the posterior of the joint distribution is similar but involves a bit more complexity. First, recall the discussion on the joint distribution of $\left\{A_{j}=1, D_{i}=1\right\}$ and let each event be denoted by $p_{u v}=\left\{A_{j}=u, D_{i}=v\right\} \forall u, v=0,1$. Also we will denote the vector of all possible $p_{u v}$ by $\mathrm{p}$. The posterior of this vector can be written as

$$
\begin{gathered}
P(\mathbf{p} \mid a, b, c, d) \propto P(A, B, C, D \mid \mathbf{p}) P(\mathbf{p})=\frac{N !}{a ! b ! c ! d !} p_{11}^{a} p_{01}^{c} p_{10}^{b}\left(1-p_{11}-p_{01}-p_{10}\right)^{d} \\
* \frac{\Gamma\left(\gamma_{11}+\gamma_{01}+\gamma_{10}+\gamma_{00}\right)}{\Gamma\left(\gamma_{11}\right) \Gamma\left(\gamma_{01}\right) \Gamma\left(\gamma_{10}\right) \Gamma\left(\gamma_{00}\right)} p_{11}^{\gamma_{11}-1} p_{01}^{\gamma_{01}-1} p_{10}^{\gamma_{10}-1}\left(1-p_{11}-p_{01}-p_{10}\right)^{\gamma_{00}-1}
\end{gathered}
$$

$\propto p_{11}^{a+\lambda_{11}-1} p_{01}^{c+\lambda_{01}-1} p_{10}^{b+\gamma_{10}-1}\left(1-p_{11}-p_{01}-p_{10}\right)^{d+\gamma_{00}-1}$ which is the kernel of a Dirichlet distribution with parameters $a+\gamma_{11}, b+\gamma_{10}, c+\gamma_{01}, d+\gamma_{00}$. Finally, to find the posterior in question, $P\left(A_{j}, D_{i} \mid a, b, c, d\right)$ we will use the same logic as before to get the marginal distribution $P\left(A_{j}, D_{i} \mid n_{i .}, n_{. .}-n_{i .}, n_{. j}, n_{. .}-n_{. j}\right) \sim \operatorname{Beta}\left(n_{i j}+\delta_{1}, n_{. .}-n_{i j}+\delta_{0}\right)$.

Using the properties of the beta distribution we can now obtain the moments required for calculation of the expected value and variance of the $I C$. Note that we will 
calculate the approximate expected value here to avoid the complexities of the logarithm of the probabilities.

$$
\begin{aligned}
E\left(I C_{i j}\right) & =E\left(\log _{2} \frac{P\left(A_{j}, D_{i} \mid n_{i,}, n_{. .}-n_{i, .}, n_{. j}, n-n_{. j}\right)}{P\left(A_{j} \mid n_{. j}, n_{. .}-n_{. j}\right) P\left(D_{i} \mid n_{i .}, n_{. .}-n_{i . .}\right)}\right) \\
& =E\left(\log _{2} P\left(A_{j}, D_{i} \mid n_{i .}, n_{. .}-n_{i .}, n_{. j}, n_{. .}-n_{. j}\right)\right) \\
& -E\left(\log _{2} P\left(A_{j} \mid n_{. j}, n_{. .}-n_{. j}\right)\right)-E\left(\log _{2} P\left(D_{i} \mid n_{i .}, n_{. .}-n_{i .}\right)\right) \\
& \approx \log _{2} E\left(P\left(A_{j}, D_{i} \mid n_{i .}, n_{. .}-n_{i . .}, n_{. j}, n_{. .}-n_{. j}\right)\right) \\
& -\log _{2} E\left(P\left(A_{j} \mid n_{. j}, n_{. .}-n_{. j}\right)\right)-\log _{2} E\left(P\left(D_{i} \mid n_{i .}, n_{. .}-n_{i .}\right)\right) \\
\text { (3.1.2) } & E\left(I C_{i j}\right) \approx \log _{2} \frac{\delta_{1}+n_{i j}}{\delta_{1}+\delta_{0}+n_{. .}}-\log _{2} \frac{\alpha_{1}+n_{. j}}{\alpha_{1}+\alpha_{0}+n_{. .}}-\log _{2} \frac{\beta_{1}+n_{i .}}{\beta_{1}+\beta_{0}+n_{. .}}
\end{aligned}
$$

We can also calculate an approximate variance using the delta method under the assumption of the independence of $A_{j}$ and $D_{i}$. This assumption allows the logarithmic variance to be expressed as

$$
\begin{aligned}
V\left(I C_{i j}\right) & =V\left(\log _{2} \frac{P\left(A_{j}, D_{i} \mid n_{i .}, n_{. .}-n_{i . .}, n_{. j}, n_{. .}-n_{. j}\right)}{P\left(A_{j} \mid n_{. j}, n_{. .}-n_{. j}\right) P\left(D_{i} \mid n_{i . .}, n_{. .}-n_{i . .}\right)}\right) \\
& =V\left(\log _{2} P\left(A_{j}, D_{i} \mid n_{i .}, n_{. .}-n_{i . .}, n_{. j}, n_{. .}-n_{. j}\right)\right) \\
& +V\left(\log _{2} P\left(A_{j} \mid n_{. j}, n_{. .}-n_{. j}\right)\right)+V\left(\log _{2} P\left(D_{i} \mid n_{i .}, n_{. .}-n_{i . .}\right)\right)
\end{aligned}
$$

This form allows us to use the delta method (1.4.3) to derive the individual terms. For this derivation we will represent the posterior distributions 
by $P_{A D}=P\left(A_{j}, D_{i} \mid n_{i .}, n_{. .}-n_{i . .}, n_{. j}, n_{. .}-n_{. j}\right), P_{A}=P\left(A_{j} \mid n_{. j}, n_{. .}-n_{. j}\right)$ and

$P_{D}=P\left(D_{i} \mid n_{i,}, n_{. .}-n_{i . .}\right)$. Also note that the estimates of these random variables will be

represented as $\hat{P}_{A D}=E\left(P_{A D}\right), \hat{P}_{A}=E\left(P_{A}\right)$ and $\hat{P}_{D}=E\left(P_{D}\right)$.

$$
\begin{aligned}
& V\left(\log _{2} P_{A D}\right) \approx\left[\frac{\partial \log _{2} P_{A D}}{\partial P_{A D}}\right]_{\hat{P}_{A D}}^{2} V\left(P_{A D}\right) \\
& =\frac{1}{\left(\ln (2) \hat{P}_{A D}\right)^{2}} \frac{\left(n_{i j}+\delta_{1}\right)\left(n_{. .}-n_{i j}+\delta_{0}\right)}{\left(n_{i j}+\delta_{1}+n_{. .}-n_{i j}+\delta_{0}\right)^{2}\left(n_{i j}+\delta_{1}+n_{. .}-n_{i j}+\delta_{0}+1\right)} \\
& =\frac{\left(\delta_{1}+n+\delta_{0}\right)^{2}}{\left(\ln (2)\left(n_{i j}+\delta_{1}\right)\right)^{2}} \frac{\left(n_{i j}+\delta_{1}\right)\left(n-n_{i j}+\delta_{0}\right)}{\left(\delta_{1}+n_{. .}+\delta_{0}\right)^{2}\left(\delta_{1}+n_{. .}+\delta_{0}+1\right)}=\frac{1}{2 \ln 2} \frac{\left(n-n_{i j}+\delta_{0}\right)}{\left(n_{i j}+\delta_{1}\right)\left(\delta_{1}+n_{. .}+\delta_{0}+1\right)} \\
& V\left(\log _{2} P_{A}\right) \approx\left[\frac{\partial \log _{2} P_{A}}{\partial P_{A}}\right]_{\hat{P}_{A}}^{2} V\left(P_{A}\right)=\frac{1}{\left(\ln (2) \hat{P}_{A}\right)^{2}} \frac{\left(n_{. j}+\alpha_{1}\right)\left(n_{. .}-n_{. j}+\alpha_{0}\right)}{\left(n_{. .}+\alpha_{1}+\alpha_{0}\right)^{2}\left(n_{. .}+\alpha_{1}+\alpha_{0}+1\right)} \\
& =\frac{\left(n_{. .}+\alpha_{1}+\alpha_{0}\right)^{2}}{\left(\ln (2)\left(n_{. j}+\alpha_{1}\right)\right)^{2}} \frac{\left(n_{. j}+\alpha_{1}\right)\left(n_{. .}-n_{. j}+\alpha_{0}\right)}{\left(n_{. .}+\alpha_{1}+\alpha_{0}\right)^{2}\left(n_{. .}+\alpha_{1}+\alpha_{0}+1\right)}=\frac{1}{2 \ln 2} \frac{\left(n_{. .}-n_{. j}+\alpha_{0}\right)}{\left(n_{. j}+\alpha_{1}\right)\left(n_{. .}+\alpha_{1}+\alpha_{0}+1\right)} \\
& V\left(\log _{2} P_{D}\right) \approx\left[\frac{\partial \log _{2} P_{D}}{\partial P_{D}}\right]_{\hat{P}_{D}}^{2} V\left(P_{D}\right)=\frac{1}{\left(\ln (2) \hat{P}_{D}\right)^{2}} \frac{\left(n_{i .}+\beta_{1}\right)\left(n_{. .}-n_{i .}+\beta_{0}\right)}{\left(n+\beta_{1}+\beta_{0}\right)^{2}\left(n_{. .}+\beta_{1}+\beta_{0}+1\right)} \\
& =\frac{\left(n+\beta_{1}+\beta_{0}\right)^{2}}{\left(\ln (2)\left(n_{i .}+\beta_{1}\right)\right)^{2}} \frac{\left(n_{i .}+\beta_{1}\right)\left(n_{. .}-n_{i .}+\beta_{0}\right)}{\left(n_{. .}+\beta_{1}+\beta_{0}\right)^{2}\left(n_{. .}+\beta_{1}+\beta_{0}+1\right)}=\frac{1}{2 \ln 2} \frac{\left(n_{. .}-n_{i .}+\beta_{0}\right)}{\left(n_{i .}+\beta_{1}\right)\left(n_{. .}+\beta_{1}+\beta_{0}+1\right)}
\end{aligned}
$$

Thus, (3.1.3)

$$
\begin{aligned}
& V(I C) \approx \\
& \frac{1}{2 \ln 2}\left(\frac{\alpha_{0}+n_{. .}-n_{i .}}{\left(\alpha_{1}+n_{i .}\right)\left(\alpha_{0}+\alpha_{1}+n_{. .}+1\right)}+\frac{\beta_{0}+n_{. .}-n_{. j}}{\left(\beta_{1}+n_{. j}\right)\left(\beta_{0}+\beta_{1}+n_{. .}+1\right)}+\frac{\delta_{0}+n_{. .}-n_{i j}}{\left(\delta_{1}+n_{i j}\right)\left(\delta_{0}+\delta_{1}+n_{. .}+1\right)}\right)
\end{aligned}
$$


Gould (Gould, 2003) proposed another method of determining these moments using the moment generating function technique. To begin we will examine a generalization of this process by letting $Y=\ln (X)$ where $X \sim \operatorname{Beta}(\alpha, \beta)$ :

$$
\begin{aligned}
& m_{Y}(t)=m_{\ln X}(t)=E\left(e^{t \ln X}\right)=E\left(X^{t}\right) \\
& =\frac{\Gamma(\alpha+\beta)}{\Gamma(\alpha) \Gamma(\beta)} \int x^{t} x^{\alpha-1}(1-x)^{\beta-1} d x=\frac{\Gamma(\alpha+\beta)}{\Gamma(\alpha) \Gamma(\beta)} \frac{\Gamma(\alpha+t) \Gamma(\beta)}{\Gamma(\alpha+\beta+t)}
\end{aligned}
$$

Thus, $m_{Y}(t)=\frac{\Gamma(\alpha+t) \Gamma(\alpha+\beta)}{\Gamma(\alpha+\beta+t) \Gamma(\alpha)}$ and by the properties of moment generating functions we can calculate expressions for the expectation and variance of $Y=\ln (X)$.

$$
\begin{gathered}
E(\ln (X))=E(Y)=m_{Y}^{\prime}(0)=\left.\frac{\partial}{\partial t} \frac{\Gamma(\alpha+t) \Gamma(\alpha+\beta)}{\Gamma(\alpha+\beta+t) \Gamma(\alpha)}\right|_{t=0}=\left.\frac{\Gamma(\alpha+\beta)}{\Gamma(\alpha)} \frac{\partial}{\partial t} \frac{\Gamma(\alpha+t)}{\Gamma(\alpha+\beta+t)}\right|_{t=0} \\
=\frac{\Gamma(\alpha+\beta)}{\Gamma(\alpha)} \frac{\Gamma^{\prime}(\alpha) \Gamma(\alpha+\beta)-\Gamma^{\prime}(\alpha+\beta) \Gamma(\alpha)}{(\Gamma(\alpha+\beta))^{2}}=\frac{\Gamma^{\prime}(\alpha)}{\Gamma(\alpha)}-\frac{\Gamma^{\prime}(\alpha+\beta)}{\Gamma(\alpha+\beta)} \\
(3.1 .4) \quad E(\ln (X))=\Psi(\alpha)-\Psi(\alpha+\beta)
\end{gathered}
$$

such that $\Psi(x)=d \ln (\Gamma(x)) / d x$.

$$
\begin{aligned}
& V(\ln (X))=V(Y)=E\left(Y^{2}\right)-[E(Y)]^{2}=m_{Y}^{\prime \prime}(t)-(\Psi(\alpha)-\Psi(\alpha+\beta)) \text { where } \\
& m_{Y}^{\prime \prime}(t)=\left.\frac{\Gamma(\alpha+\beta)}{\Gamma(\alpha)} \frac{\partial}{\partial t} \frac{\Gamma^{\prime}(\alpha+t) \Gamma(\alpha+\beta+t)-\Gamma^{\prime}(\alpha+\beta+t) \Gamma(\alpha+t)}{(\Gamma(\alpha+\beta+t))^{2}}\right|_{t=0} \\
& =\frac{\Gamma(\alpha+\beta)}{\Gamma(\alpha)} \frac{\partial}{\partial t} \frac{\Gamma^{\prime}(\alpha+t)}{\Gamma(\alpha+\beta+t)}-\left.\Psi(\alpha+\beta+t) \frac{\Gamma(\alpha+t)}{\Gamma(\alpha+\beta+t)}\right|_{t=0} \\
& =\frac{\Gamma(\alpha+\beta)}{\Gamma(\alpha)}\left[\frac{\Gamma^{\prime \prime}(\alpha) \Gamma(\alpha+\beta)-\Gamma^{\prime}(\alpha+\beta) \Gamma^{\prime}(\alpha)}{(\Gamma(\alpha+\beta))^{2}}-\left(\left.\Psi(\alpha+\beta) \frac{\partial}{\partial t} \frac{\Gamma(\alpha+t)}{\Gamma(\alpha+\beta+t)}\right|_{t=0}+\frac{\Gamma(\alpha)}{\Gamma(\alpha+\beta)} \Psi^{\prime}(\alpha+\beta)\right)\right]
\end{aligned}
$$




$$
\begin{aligned}
& =\frac{\Gamma^{\prime \prime}(\alpha)}{\Gamma(\alpha)}-\Psi(\alpha+\beta) \Psi(\alpha)-\left(\frac{\Gamma(\alpha+\beta)}{\Gamma(\alpha)} \Psi(\alpha+\beta) \frac{\Gamma^{\prime}(\alpha) \Gamma(\alpha+\beta)-\Gamma^{\prime}(\alpha+\beta) \Gamma(\alpha)}{(\Gamma(\alpha+\beta))^{2}}+\Psi^{\prime}(\alpha+\beta)\right) \\
& =\frac{\Gamma^{\prime \prime}(\alpha)}{\Gamma(\alpha)}-\Psi(\alpha+\beta) \Psi(\alpha)-\Psi(\alpha+\beta) \Psi(\alpha)+(\Psi(\alpha+\beta))^{2}-\Psi^{\prime}(\alpha+\beta) \\
& =\Psi^{\prime}(\alpha)+\Psi^{2}(\alpha)-\Psi(\alpha+\beta) \Psi(\alpha)-\Psi(\alpha+\beta) \Psi(\alpha)+(\Psi(\alpha+\beta))^{2}-\Psi^{\prime}(\alpha+\beta) \text { since } \\
& \frac{\Gamma^{\prime \prime}(\alpha)}{\Gamma(\alpha)}=\frac{[\Psi(\alpha) \Gamma(\alpha)]^{\prime}}{\Gamma(\alpha)}=\frac{\Psi^{\prime}(\alpha) \Gamma(\alpha)+\Psi(\alpha) \Gamma^{\prime \prime}(\alpha)}{\Gamma(\alpha)}=\Psi^{\prime}(\alpha)+\Psi^{2}(\alpha)
\end{aligned}
$$

Thus, $V(\ln (X))=\Psi^{\prime}(\alpha)+\Psi^{2}(\alpha)-\Psi(\alpha+\beta) \Psi(\alpha)-\Psi(\alpha+\beta) \Psi(\alpha)$

$$
\begin{gathered}
+(\Psi(\alpha+\beta))^{2}-\Psi^{\prime}(\alpha+\beta)-(\Psi(\alpha)-\Psi(\alpha+\beta))^{2} \\
V(\ln (X))=\Psi^{\prime}(\alpha)-\Psi^{\prime}(\alpha+\beta)
\end{gathered}
$$

where $\Psi(x)=d \ln (\Gamma(x)) / d x \quad \Psi^{\prime}(x)=d \Psi(x) / d x$.

For our application, we have three terms to compute using this moment generating function such that each term is in the form $Y=\ln (X) \operatorname{such}$ that $X \sim \operatorname{Beta}(\alpha, \beta)$ : $E(I C)=E\left(\log _{2} \frac{P_{A D}}{P_{A} P_{D}}\right)=\frac{1}{\ln 2}\left[E\left(\ln \left(P_{A D}\right)\right)-E\left(\ln \left(P_{A}\right)\right)-E\left(\ln \left(P_{D}\right)\right)\right]$. Thus, substituting the appropriate parameters into the expressions derived for $E(\ln (X))(3.1 .4)$ and $V(\ln (X))(3.1 .5)$ we get the following expressions which we will mark as (3.1.6) and (3.1.7) respectively. 


$$
\begin{aligned}
E(I C)= & \frac{1}{\ln (2)}\left(\Psi\left(\delta_{1}+n_{i j}\right)-\Psi\left(\delta_{0}+\delta_{1}+N\right)\right. \\
& -\left[\Psi\left(\alpha_{1}+n_{i .}\right)-\Psi\left(\alpha_{0}+\alpha_{1}+N\right)+\Psi\left(\beta_{1}+n_{. j}\right)-\Psi\left(\beta_{1}+\beta_{0}+N\right)\right] \\
V(I C)= & \frac{1}{\ln ^{2}(2)}\left(\Psi^{\prime}\left(\delta_{1}+n_{i j}\right)-\Psi^{\prime}\left(\delta_{0}+\delta_{1}+N\right)\right. \\
& \left.+\left[\Psi^{\prime}\left(\alpha_{1}+n_{i .}\right)-\Psi^{\prime}\left(\alpha_{0}+\alpha_{1}+N\right)+\Psi^{\prime}\left(\beta_{1}+n_{. j}\right)-\Psi^{\prime}\left(\beta_{1}+\beta_{0}+N\right)\right]\right)
\end{aligned}
$$

An approximate standard normal confidence interval can now be formed using these moments. A variety of authors have used this interval with a signal detection criterion of a lower bound greater than zero. Bate et al. (Bate et al., 1998) proposed a different criteria, keeping in mind a neural network model. They remove a randomly selected subset of the data, known as a test set, and then create a confidence interval using the remainder. After, they rejoin the two subsets and calculate another confidence interval. If the addition of data causes the lower bound to cross the signal detection threshold of zero, a signal is perceived. In practice, this method takes on the form of quarterly scans which are done on these databases and the confidence intervals get smaller and the estimates of the information component are less variable. This really accentuates the advantages of this method since the trends are more noticeable over time and thus dangerous ADR's are preventable.

Finally, potential dangerous interactions between drugs and ADRs that do not have a great deal of visibility may not be detected with small sample sizes. The WHO method lessens the necessity for larger samples by applying priors to the various components of the IC. However, there are dangers in this method. If our choice of prior is considerably off, a problem will occur that may require an extremely large sample to uncover. 
Fortunately, the decision to use a beta distribution seems logical due to the relationship it has with the binomial distribution, which is often more than likely appropriate due to the type of data under discussion. 


\subsection{Empirical Bayesian Screening}

Empirical Bayes screening focuses on the ratio of the unknown mean of the random variable associated with the drug-ADR pair and the expected count for the cell in question, specifically

$$
\lambda_{i j}=\mu_{i j} / E_{i j}=E\left(n_{i j}\right) / E_{i j}
$$

where $E_{i j}=\frac{n_{i .} n_{. j}}{n}$, and $n_{i j} \sim \operatorname{Poisson}\left(\mu_{i j}\right)$. It seems reasonable that if $\lambda_{i j}>1$, then we have a signal, since $E\left(n_{i j}\right)>E_{i j}$. However, several issues warrant discussion. First of all, the value of $\lambda_{i j}$ is not representative of the level of visibility in the data set. For example, an observed $n_{i j}=1200$ with an $E_{i j}=1000$ signifies a different type of signal than a value of $n_{i j}=12$ with an $E_{i j}=10$, even though their $\lambda_{i j}$ values will be the same. As with the WHO method, sampling variability, particularly in the small sample case, is an issue and, as such, similar Bayesian measures are taken.

A prior distribution is applied to $\lambda_{i j}$ in the form of a mixture of gamma distributions, where the gamma distribution is defined as $G_{i}(\lambda)=\frac{\beta_{i}^{\alpha_{i}} \lambda^{\alpha_{i}-1} e^{-\lambda \beta_{i}}}{\Gamma\left(\alpha_{i}\right)}$. The mixture can be defined as 


$$
f\left(\lambda_{i j} ; \alpha_{1}, \beta_{1}, \alpha_{2}, \beta_{2}, P\right)=P^{*} G_{1}\left(\lambda_{i j} ; \alpha_{1}, \beta_{1}\right)+(1-P)^{*} G_{2}\left(\lambda_{i j} ; \alpha_{2}, \beta_{2}\right)
$$

where $P$ represents the probability of lambda being in the first component of the mixture or, $P=P\left(\lambda_{i j} \in G_{1}\right)$. This distribution was chosen for several reasons. Of primary importance is the fact that the gamma distribution has a conjugate relationship with the Poisson distribution. In addition by invoking a prior distribution with a large number of parameters, it enhances the flexibility of the distribution function, thereby allowing for a more precise posterior. The posterior in question is conditional on the random variable $n_{i j}$, or the observed count in the cell in question. To simplify the calculation of the posterior distribution, the marginal distribution of $n_{i j}$ is derived by integrating $\lambda_{i j}$ out of the joint distribution, $n_{i j}, \lambda_{i j}$.

$$
\begin{aligned}
& h\left(n_{i j}, \lambda_{i j}\right)=h\left(n_{i j} ; \mu_{i j}\right)\left[P^{*} G_{1}\left(\lambda_{i j} ; \alpha_{1}, \beta_{1}\right)+(1-P)^{*} G_{2} *\left(\lambda_{i j} ; \alpha_{2}, \beta_{2}\right)\right] \\
& =P^{*} h\left(n_{i j} ; \mu_{i j}\right) * G_{1}\left(\lambda_{i j} ; \alpha_{1}, \beta_{1}\right)+(1-P)^{*} h\left(n_{i j} ; \mu_{i j}\right) * G_{2}\left(\lambda_{i j} ; \alpha_{2}, \beta_{2}\right) \\
& f\left(n_{i j}\right)=P * \int_{0}^{\infty} h\left(n_{i j} ; \mu_{i j}\right) * G_{1}\left(\lambda_{i j} ; \alpha_{1}, \beta_{1}\right) d \lambda_{i j}+(1-P)^{*} \int_{0}^{\infty} h\left(n_{i j} ; \mu_{i j}\right) * G_{2}\left(\lambda_{i j} ; \alpha_{2}, \beta_{2}\right) d \lambda_{i j}
\end{aligned}
$$

To complete this proof we will only solve one of the integrals since they are essentially the same. For the purposes of this solution we will drop the subscript and recall that

$$
\begin{aligned}
& \mu_{i j}=\lambda_{i j} E_{i j} . \\
& \int_{0}^{\infty} h(n ; \mu)^{*} G(\lambda ; \alpha, \beta) d \lambda=\int_{0}^{\infty} \frac{e^{-\lambda E}(\lambda E)^{n}}{n !} \frac{\lambda^{\alpha-1} \beta^{\alpha} e^{-\lambda \beta}}{\Gamma(\alpha)} d \lambda
\end{aligned}
$$




$$
\begin{aligned}
& =\frac{\beta^{\alpha} E^{n}}{\Gamma(\alpha) n !} \int_{0}^{\infty} e^{-\lambda(E+\beta)} \lambda^{n+\alpha-1} d \lambda=\frac{\beta^{\alpha} E^{n}}{\Gamma(\alpha) n !} \frac{\Gamma(n+\alpha)}{(E+\beta)^{n+\alpha}} \\
& =\left(\frac{\beta}{E+\beta}\right)^{\alpha}\left(\frac{E}{E+\beta}\right)^{n} \frac{\Gamma(n+\alpha)}{\Gamma(\alpha) n !}=N B_{k}\left(n_{i j} ; \alpha_{k}, \beta_{k}, E_{i j}\right)
\end{aligned}
$$

Thus, the marginal distribution of $n_{i j}$ is a mixture of negative binomials of the form

$$
f\left(n_{i j}\right)=P^{*} N B_{1}\left(n_{i j} ; \alpha_{1}, \beta_{1}, E_{i j}\right)+(1-P) * N B_{2}\left(n_{i j} ; \alpha_{2}, \beta_{2}, E_{i j}\right)
$$

Note that $P=P\left(\lambda \in N B_{1}\right)$ as it simply represents a weight describing how much of $\lambda_{i j}$ is in each piece of the mixture.

This distribution is used to search for the values of the parameters, denoted as $\theta=\left\{\alpha_{1}, \beta_{1}, \alpha_{2}, \beta_{2}, P\right\}$, by maximizing the likelihood function of $f\left(n_{i j}\right)$. The negative binomial distribution is a nice fit since it can be defined as the number of trials needed to achieve $n$ successes with a given success probability. Also, the negative binomial gives a larger tolerance to highly variable data sets. Since the mean of the Poisson distribution is equal to its variance, it may not be able to describe the extra-Poisson variation often seen in pharmacogivilance data.

A variety of methods can be used to search for the parameters of this negative binomial, including the Newton-Raphson algorithm. Consider the problem of maximizing $f(\mathrm{p})$ with respect to $\hat{\mathrm{p}}$ where $f(\mathrm{p})$ is a differentiable density function. The 
second order Taylor Series approximation (1.4.1) about $\mathbf{p}=\mathbf{P}$ where $\mathbf{p}=\left(p_{1}, \ldots, p_{n}\right)$ is $f(\hat{\mathbf{p}}) \approx f\left(\mathrm{P}^{(t)}\right)+\Delta^{(t)}(\mathbf{p})\left(\hat{\mathbf{p}}-\mathbf{P}^{(t)}\right)+\frac{\left(\hat{\mathbf{p}}-\mathbf{P}^{(t)}\right) \mathrm{H}^{(t)}\left(\hat{\mathbf{p}}-\mathbf{P}^{(t)}\right)^{T}}{2}$ such that $t$ represents the current number of iterations. The Newton-Raphson is an iterative method for solving the value of $\hat{\mathbf{p}}$ that maximizes $f(\mathrm{p})$. Taking the derivative of the Taylor series expansion and setting it to zero yields: $\frac{\partial f(\hat{\mathbf{p}})}{\partial \hat{\mathbf{p}}} \approx \Delta^{(t)}+\mathrm{H}^{(t)}\left(\hat{\mathrm{p}}-\mathrm{P}^{(t)}\right)=0$. Solving for $\hat{\mathbf{p}}$ gives us the next step in the iteration, $\mathbf{P}^{(t+1)}=\mathbf{P}^{(t)}-\left[\mathrm{H}^{(t)}\right]^{-1} \Delta^{(t)}$. At some point the difference in the estimates from step to step are small enough that it is reasonable to stop and conclude that the elements of the vector $\hat{\mathbf{p}}$ maximize $f(\mathbf{p})$. Usually the size of this step is specified by the investigator.

A number of other nonlinear maximization methods can be used for the parameter search. Since the parameters in question are the location and scale parameters of two Gamma distributions, as well a weight, the ranges of these values are problematic. They are restricted to $[0, \infty)$ for the Gamma parameters and $[0,1]$ for the weight. By applying a transformation to the parameters to be estimated, we can extend the parameter space to the entire real line $(-\infty,+\infty)$, which will remove some of the issues surrounding appropriate maximization of the density function. Specifically, we apply the transformation $\theta_{1}=\ln \left(\alpha_{1}\right), \theta_{2}=\ln \left(\beta_{1}\right), \theta_{3}=\ln \left(\alpha_{2}\right), \theta_{4}=\ln \left(\beta_{2}\right)$ and $\theta_{5}=\ln \left(\frac{P}{1-P}\right)$. By solving the transformed derivatives and applying the Newton-Raphson method, we can 
obtain the maximum likelihood estimates of the transformed parameters. Note, that this transformation is unnecessary for maximization methods where the ranges of each parameter can be restricted by the investigator. Many of these functions are available in well-known statistical computing packages such as $\mathrm{R}$ or S-Plus.

Once the search for $\theta=\left(\alpha_{1}, \beta_{2}, \alpha_{1}, \beta_{2}, P\right)^{T}$ is complete, our next goal is to determine the posterior distribution of $\lambda_{i j} \mid n_{i j}$. By simply conditioning on the prior distribution $f\left(\lambda_{i j}\right)$ we get the expression $f\left(\lambda_{i j} \mid n_{i j}\right)=P\left(\lambda_{i j} \in G_{1} \mid n_{i j}\right) * G_{1}\left(\lambda_{i j} \mid n_{i j}\right)+\left[1-P\left(\lambda_{i j} \in G_{2} \mid n_{i j}\right)\right]^{*} G_{2}\left(\lambda_{i j} ; n_{i j}\right)$. Thus we need expressions for $P\left(\lambda_{i j} \in G_{1} \mid n_{i j}\right)$ which we will define as $Q_{n}$, and $G_{i}\left(\lambda_{i j} \mid n_{i j}\right)$.

If we define $\xi_{k}$ to be the event $\left\{\lambda_{i j} \in G_{k}\right\}$, then $Q_{n}=P\left(\xi_{k} \mid n_{i j}\right)=\frac{P\left(n_{i j} \mid \xi_{k}\right) P\left(\xi_{k}\right)}{P\left(n_{i j}\right)}=\frac{P\left(n_{i j} \mid \xi_{k}\right)}{P\left(n_{i j}\right)}$ and,

$$
Q_{n}=\frac{P^{*} N B\left(n \mid \alpha_{1}, \beta_{1}, E\right)}{P^{*} N B\left(n \mid \alpha_{1}, \beta_{1}, E\right)+(1-P)^{*} N B\left(n \mid \alpha_{2}, \beta_{2}, E\right)}
$$

For the posterior of the gammas we will once again only derive one of them since they are the same distribution. 
$G_{k}\left(\lambda_{i j} \mid n_{i j}\right) \propto p\left(n_{i j} \mid \lambda_{i j} E_{i j}\right) G_{k}\left(\lambda_{i j} \mid \alpha_{k}, \beta_{k}\right)=\frac{e^{-\lambda_{i j} E_{i j}}\left(\lambda_{i j} E_{i j}\right)^{n_{i j}}}{n_{i j} !} \frac{\lambda^{\alpha_{k}-1} e^{-\lambda \beta_{k}} \beta_{k}^{\alpha_{k}}}{\Gamma\left(\alpha_{k}\right)}$

$\propto \lambda_{i j}^{n_{i j}+\alpha_{k}-1} e^{-\lambda_{i j}\left(E_{i j}+\beta_{k}\right)}$, which is the kernel of a Gamma distribution with parameters

$n_{i j}+\alpha_{k}, \beta_{k}+E_{i j}$

We can now introduce the posterior distribution of $\lambda_{i j} \mid n_{i j}$ as

$$
f\left(\lambda_{i j} \mid n_{i j}\right)=Q_{n} G_{1}\left(\lambda_{i j} \mid \alpha_{1}+n_{i j}, \beta_{1}+E_{i j}\right)+\left(1-Q_{n}\right) G_{2}\left(\lambda_{i j} \mid \alpha_{2}+n_{i j}, \beta_{2}+E_{i j}\right)
$$

The statistic we need for signal detection is simply the expectation of this distribution.

This is easily obtained as

$E\left(\lambda_{i j} \mid n_{i j}\right)=Q_{n} E\left[G_{1}\left(\alpha_{1}+n_{i j}, \beta_{1}+E_{i j}\right)\right]+\left(1-Q_{n}\right) E\left[G_{2}\left(\alpha_{2}+n_{i j}, \beta_{2}+E_{i j}\right)\right]$

$$
E\left(\lambda_{i j} \mid n_{i j}\right)=Q_{n} \frac{\alpha_{1}+n_{i j}}{\beta_{1}+E_{i j}}+\left(1-Q_{n}\right) \frac{\alpha_{2}+n_{i j}}{\beta_{1}+E_{i j}}
$$

using basic properties of the gamma distribution and expectation. It is convenient to find the logarithmic expectation because it will lower the variance of the estimate, and so we will begin by using the change of variable technique.

Let $z=\ln (\lambda) \Rightarrow \lambda=e^{z}$, thus

$$
\begin{aligned}
& f_{Z}(z)=f_{\Lambda \mid n}\left(e^{z}\right)\left|\frac{d e^{z}}{d z}\right|=e^{z_{i j}}\left[Q_{n} G_{1}\left(e^{z_{i j}} \mid \alpha_{1}+n_{i j}, \beta_{1}+E_{i j}\right)+\left(1-Q_{n}\right) G_{2}\left(e^{z_{i j}} \mid \alpha_{2}+n_{i j}, \beta_{2}+E_{i j}\right)\right] \\
& =Q_{n} G_{1}\left(e^{z_{i j}} \mid \alpha_{1}+n_{i j}, \beta_{1}+E_{i j}\right) e^{z_{i j}}+\left(1-Q_{n}\right) G_{2}\left(e^{z_{i j}} \mid \alpha_{2}+n_{i j}, \beta_{2}+E_{i j}\right) e^{z_{i j}}
\end{aligned}
$$


Note that if $X \sim \Gamma(\alpha, \beta)$ and $Y=\ln (X)$ then the log-gamma distribution can be written as $f_{Y}(y)=\frac{e^{y(\alpha-1)} e^{-e^{y} \beta} \beta^{\alpha}}{\Gamma(\alpha)} e^{y}$, which is the same as $G\left(e^{y} \mid \alpha, \beta\right) e^{y}$. Thus, each term in $f_{Z}$ is a log gamma with the posterior parameters derived in $\lambda \mid n$, or $f\left(\ln \left(\lambda_{i j}\right) \mid n_{i j}\right)=Q_{n} L G_{1}\left(\lambda_{i j} \mid \alpha_{1}+n_{i j}, \beta_{1}+E_{i j}\right)+\left(1-Q_{n}\right) L G_{2}\left(\lambda_{i j} \mid \alpha_{2}+n_{i j}, \beta_{2}+E_{i j}\right)$ where $L G(\lambda \mid \alpha, \beta)=\frac{\exp \left(\lambda \alpha-e^{\lambda} \beta\right) \beta^{\alpha}}{\Gamma(\alpha)}$

Interestingly enough, we get the same result if we condition on the log-gamma distribution, as opposed to finding the log of the posterior. Once again, let $n \sim \operatorname{Poisson}(\mu)$ and $\lambda \sim \Gamma(\alpha, \beta)$. However, we now want the distribution of $Y=\ln (\lambda)$ which implies that we can rewrite the Poisson as $n \sim$ Poisson $\left(e^{y} E\right)$ since $\lambda=\frac{\mu}{E}$. Thus, we have $h(y \mid n) \propto p\left(n \mid e^{y} E\right) L G(y \mid \alpha, \beta)=\frac{\exp \left(-e^{y} E\right)\left(e^{y} E\right)^{n}}{n !} \frac{\exp \left(y \alpha-e^{y} \beta\right) \beta^{\alpha}}{\Gamma(\alpha)}$ $\propto \exp \left(-e^{y}(E+\beta)\right) \exp (y(\alpha+n))$ which is the kernel of a log-gamma with parameters $n+\alpha, \beta+E$.

We can now derive the expectation of $L G(\lambda \mid \alpha, \beta)$ using the moment generating function technique. 
If we consider $X \sim \Gamma(\alpha, \beta)$, then

$m_{\ln X}(t)=E\left(e^{t \ln X}\right)=E\left(X^{t}\right)=\int x^{t} \frac{x^{\alpha-1} \beta^{\alpha} e^{-x / \beta}}{\Gamma(\alpha)} d x=\frac{\Gamma(\alpha+t)}{\Gamma(\alpha) \beta^{t}}$. Differentiating this

function about zero gives us the expectation.

$$
\begin{aligned}
& E(\ln X)=m_{\ln X}^{\prime}(0)=\left.\frac{1}{\Gamma(\alpha)} \frac{\delta}{\delta t} \frac{\Gamma(\alpha+t)}{\beta^{t}}\right|_{t=0}=\left.\frac{1}{\Gamma(\alpha)} \frac{\Gamma^{\prime}(\alpha+t) \beta^{t}-\ln (\beta) \beta^{t} \Gamma(\alpha+t)}{\beta^{2 t}}\right|_{t=0} \\
& =\frac{1}{\Gamma(\alpha)} \Gamma^{\prime}(\alpha)-\ln (\beta) \Gamma(\alpha)=\Psi(\alpha)-\ln (\beta) \text { where } \Psi(\alpha)=\frac{\Gamma^{\prime}(\alpha)}{\Gamma(\alpha)} .
\end{aligned}
$$

We can also find the variance of $\ln X$ using this technique.

$$
\begin{gathered}
\operatorname{Var}(\ln X)=E\left(X^{2}\right)-(E X)^{2}=m_{\ln X}^{\prime \prime}(0)-(\Psi(\alpha)-\ln (\beta))^{2} \\
m_{\ln X}^{\prime \prime}(0)=\left.\frac{1}{\Gamma(\alpha)} \frac{d^{2}}{d t^{2}} \frac{\Gamma(\alpha+t)}{\beta^{t}}\right|_{t=0}=\left.\frac{1}{\Gamma(\alpha)} \frac{d}{d t} \frac{\Gamma^{\prime}(\alpha+t)-\Gamma(\alpha+t) \ln (\beta)}{\beta^{t}}\right|_{t=0} \\
=\frac{\Gamma^{\prime \prime}(\alpha)}{\Gamma(\alpha)}-2 \ln \beta \Psi(\alpha)+2 \ln \beta
\end{gathered}
$$

Thus, $\operatorname{Var}(\ln X)=\frac{\Gamma^{\prime \prime}(\alpha)}{\Gamma(\alpha)}-2 \ln \beta \Psi(\alpha)+2 \ln \beta-(\Psi(\alpha)-\ln (\beta))^{2}$

$$
\begin{aligned}
& =\frac{\Gamma^{\prime \prime}(\alpha)}{\Gamma(\alpha)}-2 \ln \beta \Psi(\alpha)+2 \ln \beta-\Psi^{2}(\alpha)-2 \ln \beta+2 \ln \beta \Psi(\alpha) \\
& =\frac{\Gamma^{\prime \prime}(\alpha)}{\Gamma(\alpha)}-\Psi^{2}(\alpha)=\frac{d}{d \alpha} \frac{\Gamma^{\prime}(\alpha)}{\Gamma(\alpha)}=\Psi^{\prime}(\alpha) \text { which is the trigamma function. }
\end{aligned}
$$


Resubstituting the appropriate parameters into the formula for the logarithmic expectation gives us

$$
\begin{aligned}
& E\left(\ln \left(\lambda_{i j}\right) \mid n_{i j}\right) \\
& =Q_{n}\left[\Psi\left(\alpha_{1}+n_{i j}\right)-\ln \left(\beta_{1}+E_{i j}\right)\right]+\left(1-Q_{n}\right)\left[\Psi\left(\alpha_{2}+n_{i j}\right)-\ln \left(\beta_{2}+E_{i j}\right)\right]
\end{aligned}
$$

Finally, the statistic known as the empirical Bayes Geometric Mean (EBGM), denoted by $\Lambda$, is the exponential of the logarithmic expectation,

$$
\Lambda=\exp (E(\ln (\lambda) \mid n))
$$

It should be noted that this method relies entirely on the outcome of the maximization of the likelihood function, which in turn will provide the form of the posterior distribution. The creator of this method, William DuMouchel (Dumouchel, 1999), has provided additional suggestions for simplifying this maximization step. The first such simplification is to condition on the set, $n \geq n^{*}$ where $n^{*}$ can be defined as a reasonable lower bound for a potential signal. For example, an observed value of zero cannot be a signal by definition so setting $n^{*}=1$, and excluding these cells from the parameter search will greatly increase the efficiency of the algorithm. The resulting conditional distributions can replace the negative binomial distributions in the likelihood and will decrease the number of cells examined in each iteration:

$$
P\left(N=n \mid N \geq n^{*}\right)=\frac{P\left(N \geq n^{*} \mid N=n\right) P(N=n)}{P\left(N \geq n^{*}\right)}=\frac{P\left(N \geq n^{*} \mid N=n\right)}{P\left(N<n^{*}\right)}=\frac{N B(n)}{1-\sum_{m=1}^{n^{*}-1} N B(m)}
$$


Another suggestion for improving the optimization step is known as data squashing, also proposed by DuMouchel (Dumouchel et al., 1999). To "squash", we partition the set of all $(n, E)$ into a group of $M$ bins of $(n, E, w)$, where the $w$ acts as a bin counter. The idea is to make the within-bin moments match the original data set by adjusting the weight, $w$. This reduces the likelihood to $M$ terms of the form:

$$
L(\theta)=\prod_{i=1}^{M}\left[P N B_{*}\left(\alpha_{1}, \beta_{1}, E_{i}\right)+(1-P) N B_{*}\left(\alpha_{2}, \beta_{2}, E_{i}\right)\right]^{w_{i}}
$$

Finally, the question of signal detection criteria arises. DuMouchel did not propose this method to be used in this manner; instead he developed it as a ranking method. Roux et al. (Roux et al., 2005) proposed a signal detection criterion that relies on the probability that the ratio $\lambda$ comes from a gamma distribution with an expected value greater than one. They define this probability as

$$
E B p=Q \delta_{1}+(1-Q) \delta_{2} \text { such that } k \in\{1,2\} \begin{cases}\delta_{k}=1 & \text { if } \frac{\alpha_{k}+n}{\beta_{k}+E}>1 \\ \delta_{k}=0 & \text { otherwise }\end{cases}
$$

The pair in question is flagged as a signal if $E B p>0.5$, or the probability that $\lambda_{i j}$ comes from a gamma with an expectation greater than one is $50 \%$.

Another form of signal criteria is similar to the WHO method. It is derived by creating a confidence interval for $\lambda$ and comparing its lower bound to one. However, this method gives us the entire posterior distribution and as such this lower bound can easily be found by looking at the quantiles of this distribution. However, Gould (Gould, 
2003) attempted to derive the variance of $\ln \left(\lambda_{i j}\right) \mid n_{i j}$ in order to facilitate the determination of such an interval. By the properties of the variance of a sum of dependant random variables, we have

$$
\begin{aligned}
& V\left(\ln \left(\lambda_{i j}\right) \mid n_{i j}\right)=V\left[Q_{n} L G_{1}\left(\lambda_{i j} \mid \alpha_{1}+n_{i j}, \beta_{1}+E_{i j}\right)+\left(1-Q_{n}\right) L G_{2}\left(\lambda_{i j} \mid \alpha_{2}+n_{i j}, \beta_{2}+E_{i j}\right)\right] \\
& =Q_{n}^{2} V\left(L G_{1}\left(\lambda_{i j} \mid \alpha_{1}+n_{i j}, \beta_{1}+E_{i j}\right)\right)+\left(1-Q_{n}\right)^{2} V\left(L G_{2}\left(\lambda_{i j} \mid \alpha_{2}+n_{i j}, \beta_{2}+E_{i j}\right)\right) \\
& +2 Q_{n}\left(1-Q_{n}\right) \operatorname{Cov}\left[L G_{1}\left(\lambda_{i j} \mid \alpha_{1}+n_{i j}, \beta_{1}+E_{i j}\right), L G_{2}\left(\lambda_{i j} \mid \alpha_{2}+n_{i j}, \beta_{2}+E_{i j}\right)\right]
\end{aligned}
$$

Note that we already have expressions for the variance of the individual loggammas, however we do not have an expression for the covariance term. Gould's equation is as follows,

$$
\begin{aligned}
& V\left(\log _{2} \lambda \mid n\right)=\ln ^{-2}(2)\left[Q_{n} \Psi^{\prime}\left(\alpha_{1}+n\right)+\left(1-Q_{n}\right) \Psi^{\prime}\left(\alpha_{2}+n\right)\right. \\
& +2 Q_{n}\left(1-Q_{n}\right)\left[\Psi\left(\alpha_{1}+n\right)-\ln \left(\beta_{1}+E\right)-\left(\Psi\left(\alpha_{2}+n\right)-\ln \left(\beta_{2}+E\right)\right)\right]^{2}
\end{aligned}
$$

and an expression is given for this covariance term. Note that the covariance expression is simply $\left[E\left(L G_{1}\left(\lambda_{i j} \mid n_{i j}\right)\right)-E\left(L G_{1}\left(\lambda_{i j} \mid n_{i j}\right)\right)\right]^{2}$. However, this appears to be incorrect based on the following. For simplicity we will let $G_{k} \sim \Gamma\left(\alpha_{k}, \beta_{k}\right) \forall k=1,2$ and $X=\ln G_{1}$ and $Y=\ln G_{2}$. By the Cauchy-Scwartz inequality we have $\operatorname{Cov}^{2}(X, Y) \leq \operatorname{Var}(X) \operatorname{Var}(Y)$ which translates to $\operatorname{Cov}^{2}(X, Y)=[E(X)-E(Y)]^{4} \leq \operatorname{Var}(X) \operatorname{Var}(Y)$ in our application.

$$
\begin{aligned}
{[E(X)} & -E(Y)]^{4}=\sum_{i=0}^{4}\left(\begin{array}{l}
4 \\
i
\end{array}\right) E(X)^{i} E(Y)^{4-i} \leq \operatorname{Var}(X) \operatorname{Var}(Y) \\
& =\left[E\left(X^{2}\right)-E(X)^{2}\right]\left[E\left(Y^{2}\right)-E(Y)^{2}\right]
\end{aligned}
$$




$$
\begin{aligned}
E(Y)^{4} & +4 E(X) E(Y)^{3}+6 E(X)^{2} E(Y)^{2}+4 E(X)^{3} E(Y)+E(X)^{4} \\
& \leq E\left(X^{2}\right) E\left(Y^{2}\right)+E(X)^{2} E(Y)^{2}-E(Y)^{2} E\left(X^{2}\right)-E\left(Y^{2}\right) E(X)^{2} \\
E(Y)^{4} & +4 E(X) E(Y)^{3}+5 E(X)^{2} E(Y)^{2}+4 E(X)^{3} E(Y)+E(X)^{4} \\
& +E(Y)^{2} E\left(X^{2}\right)+E\left(Y^{2}\right) E(X)^{2} \leq E\left(X^{2}\right) E\left(Y^{2}\right)
\end{aligned}
$$

Note that the even powers must be positive and since we know what the variance and expectation of $\mathrm{X}$ and $\mathrm{Y}$ are, we can determine the second moment, $E\left(X^{2}\right)=\operatorname{Var}(X)+E(X)^{2}$. The variance of $\mathrm{X}$ and $\mathrm{Y}$ are both the trigamma function $\Psi^{\prime}\left(\alpha_{k}\right)$, which is always positive. The squared expectation is also always positive and as such the second moments must be always positive as well. Also by Jensen's inequality we have $E\left(X^{2}\right) \geq E(X)^{2}$ which again implies that the second moment is always positive. We can apply Jensen's inequality because $[\ln (U)]^{2}$ is a convex function. Using Jensen's inequality we can continue,

$$
\begin{aligned}
& E(Y)^{4}+4 E(X) E(Y)^{3}+5 E(X)^{2} E(Y)^{2}+4 E(X)^{3} E(Y) \\
& \quad+E(X)^{4}+E(Y)^{2} E(X)^{2}+E(Y)^{2} E(X)^{2} \\
& =E(Y)^{4}+4 E(X) E(Y)^{3}+7 E(X)^{2} E(Y)^{2}+4 E(X)^{3} E(Y)+E(X)^{4} \quad \leq E\left(X^{2}\right) E\left(Y^{2}\right)
\end{aligned}
$$

The final piece of the proof is to note that although the expectations can be negative if both expectations are the same sign, the left hand side is considerably larger than the right hand side. Therefore, the covariance term proposed by Gould cannot be true under all scenarios. Finally, the weights, $Q_{n}$, are also problematic in that in Gould's 
formula they are not squared, however the known formula the variance of the coefficient of a random variable is $\operatorname{Var}(a X)=a^{2} \operatorname{Var}(X)$.

So how do we develop this term in the variance formula? Specifically we need the joint distribution, or the distribution where both marginals are our gammas, $G_{k}$. There are many methods proposed in the literature for this type of derivation and some of the more famous ones are summed in Kotz's book, "Continuous Multivariate Distributions" (Kotz et al., 2000). As an example, D'este (D'Este, 1981) attempted to create such a distribution by using Morgenstern's procedure. He proposed that we can create a system of bivariate cdfs with the following formula, $F(x, y)=G(x) H(y)[1+\lambda(1-G(x))(1-H(y))]$ such that $|\lambda| \leq 1$. Naturally, the pdf can be easily derived from this and D'Este applied this to a pair of gamma marginals. Although a great deal of information was derived such as joint moments and conditional moments, his conclusions were such that this distribution is only valid under the assumption of a low correlation. Unfortunately for our application, we are using his formula to test for correlation and as such this assumption will cause problems in the decision-making abilities of this method.

Overall, it appears to be a difficult task to determine an adequate joint distribution. Methods for deriving these sorts of distributions generally require a set of assumptions applied to the conditional distribution, either directly or indirectly. This forcing of assumption limits the generality of the bivariate distribution and as such a great deal of care must be taken in picking the appropriate method. In pharmacovigilance this 
is a problem that stems past the scope of this discussion, however there are some promising methods in the literature that can be expanded upon in the future to derive the covariance term.

Fortunately, a confidence interval is unnecessary here since we have the entire posterior distribution at our disposal. Gould (Gould, 2003) as well as others have proposed simply calculating the quantiles of the distribution in question and determining a cutoff such as 0.05 or 0.1 . In other words the solution to

$$
\alpha=\int_{0}^{\lambda_{u}} p(\lambda \mid N=n) d \lambda
$$

will give us an exact value of $\lambda$ according to the posterior distribution. This value, can be compared to the value of one or two (van Puijenbroek et al., 2002; Szarfman et al., 2002;Roux et al., 2005; Hauben et al., 2005) to determine a signal.

This method has a number of advantages. Not only does it use Bayesian techniques to better deal with small sample scenarios, it uses 'shrinkage' techniques to effectively lower the magnitude of the signal if there is a low frequency of reports, thereby facilitating the detection of weaker signals. Like the WHO method, there are the usual reservations about the adoption of a Bayesian framework. If the priors are not well chosen, the posteriors will be adversely affected. Also of note is that this estimator uses distributions that are extremely flexible based on the high number of parameters. 


\section{Chapter 4}

\section{Pearson Standardized Residuals}

We have now considered the main signal detection methods used in passive pharmacovigilance. However, this list is not exhaustive in that there exists a large number of methods used to assess independence in contingency tables. A method not currently used in pharmacovigilance is proposed in this section and will be used in the simulation study in Chapter 6.

Pearson residuals (Agresti, 2002) are a method of further examining the dependencies in contingency tables. Measures such as the Yate's chi square and Pearson's chi square produce values that speak to the overall dependency in the $2 \times 2$ table however they do not say much beyond that. Standardizing the residuals of each cell in the larger contingency table can prove useful to better understanding the individual associations and dependencies. This is done by considering that each $n_{i j}$ can be transformed through the usual standardization procedure to have similar means and variances. Also this will give us an approximately normally distributed variable, which can then be compared to a critical value to assess the strength of association of the individual cell. In this application our random variable, $n_{i j}$, is Poisson distributed with an unknown rate. To generate a residual, the general method is to subtract the mean of the Poisson from the observed data. However, the unknown rate requires an estimate and 
due to the fact that the Poisson only has one parameter we will potentially encounter over dispersion since the variance is dependent on the mean. To correct for this, Pearson suggested standardizing the residuals as follows

$$
e_{i j}=\frac{n_{i j}-E_{i j}}{\sqrt{E_{i j}}}
$$

However, $\sqrt{E_{i j}}$ is not the true variance of $n_{i j}-E_{i j}$ under $H_{0}$. This can be determined using the delta method (1.4.1). First we can express the difference as follows, $n_{i j}-E_{i j}=n_{i j}-\hat{n}_{i j}=n_{. .}\left(p_{i j}-\hat{p}_{i j}\right) \stackrel{H_{0}}{=} n_{. .}\left(p_{i j}-p_{i .} p_{. j}\right)$, where $p_{i j}=n_{i j} / n_{. .}$. Now we are left with a function of $p$ and can apply the delta method to this function to solve the asymptotic variance. If we once again treat the elements of our $2 \times 2$ table (1.3.2) as multinomial random variables with parameters $p_{i}, n_{i} \forall i=a, b, c, d$, we can use the results derived in section 2.2 to assist us in this derivation. Recall that $\sqrt{n}[g(\hat{\mathbf{p}})-g(\mathbf{p})] \stackrel{d}{\longrightarrow} N\left(0, \varphi^{T}\left[\operatorname{diag}(\mathbf{p})-\mathbf{p p}^{T}\right] \varphi\right)(2.2 .3)$ where $\mathbf{p}=\left(p_{a}, p_{b}, p_{c}, p_{d}\right)^{T}$ which is estimated by $\hat{\mathbf{p}}$ and $\varphi=\frac{d g(\mathbf{p})}{d \mathbf{p}}=\left[\frac{\partial g(\mathbf{p})}{\partial p_{a}}, \frac{\partial g(\mathbf{p})}{\partial p_{b}}, \frac{\partial g(\mathbf{p})}{\partial p_{c}}, \frac{\partial g(\mathbf{p})}{\partial p_{d}}\right]^{T}$. Thus, the asymptotic variance of $\sqrt{n}[g(\hat{\mathbf{p}})-g(\mathbf{p})]$ is $\boldsymbol{\varphi}^{T}\left[\operatorname{diag}(\mathbf{p})-\mathbf{p} \mathbf{p}^{T}\right] \boldsymbol{\varphi}=\sum_{i=a, b, c, d} p_{i} \phi_{i}^{2}-\left(\sum_{i=a, b, c, d} p_{i} \phi_{i}\right)^{2}$. For simplicity we will use a new notation for this derivation. 
Let $n_{11}=a, n_{12}=b, n_{21}=c, n_{22}=d, n=a+b+c+d$ then $\frac{n_{i j}}{n}=p_{i j} \forall i, j=1,2$ and $\mathrm{p}=\left(p_{11}, p_{12}, p_{21}, p_{22}\right)^{T}$. Thus our function can be rewritten as $n\left(p_{11}-\left(p_{11}+p_{12}\right)\left(p_{11}+p_{21}\right)\right)$ and the gradient vector is $\boldsymbol{\varphi}=\frac{d g(\mathbf{p})}{d \mathbf{p}}=n\left[\left(1-p_{1 .}-p_{.1}\right),-p_{1 .},-p_{.1}, 0\right]^{T}$. First, we will evaluate the second term in the expression:

$$
\begin{aligned}
& \left(\sum_{i, j=1}^{2} p_{i j} \phi_{i j}\right)^{2}=n^{2}\left(p_{11} \phi_{11}+p_{12} \phi_{12}+p_{21} \phi_{21}+p_{22} \phi_{22}\right)^{2} \\
& =n^{2}\left(p_{11}\left(1-p_{1 .}-p_{.1}\right)-p_{12} p_{.1}-p_{21} p_{1}\right)^{2}=n^{2} n^{2}\left(p_{11}-p_{11} p_{1 .}-p_{11} p_{.1}-p_{12} p_{.1}-p_{21} p_{1 .}\right)^{2}
\end{aligned}
$$

Note that due to the null hypothesis assumption of independence we can write $p_{i j}=p_{i .} p_{. j}$ and our expression becomes:

$$
\begin{aligned}
& \left(\sum_{i, j=1}^{2} p_{i j} \phi_{i j}\right)^{2}=n^{2}\left(p_{1 .} p_{.1}\right)^{2}\left(1-p_{1 .}-p_{.1}-p_{.2}-p_{2 .}\right)^{2}=n^{2}\left(p_{1 .} p_{.1}\right)^{2} \text { since } \\
& p_{1 .}+p_{2 .}=p_{.1}+p_{.2}=1 .
\end{aligned}
$$

For the first term,

$$
\begin{aligned}
& \sum_{i, j=1}^{2} p_{i j} \phi_{i j}^{2}=n^{2}\left(p_{11} \phi_{11}^{2}+p_{12} \phi_{12}^{2}+p_{21} \phi_{21}^{2}+p_{22} \phi_{22}^{2}\right) \\
& =n^{2}\left(p_{11}\left(1-p_{1 .}-p_{.1}\right)^{2}+p_{12} p_{.1}^{2}+p_{21} p_{1 .}^{2}\right) \stackrel{H_{0}}{=} n^{2} p_{1 .} p_{.1}\left[\left(1-p_{1 .}-p_{.1}\right)^{2}+p_{2 .} p_{1 .}+p_{.2} p_{.1}\right] \\
& =n^{2} p_{1 .} p_{.1}\left[\left(1-p_{1 .}-p_{.1}\right)^{2}+\left(1-p_{1 .}\right) p_{1 .}+\left(1-p_{.1}\right) p_{.1}\right] \\
& =n^{2} p_{1 .} p_{.1}\left[1+\left(p_{1 .}+p_{.1}\right)^{2}-2\left(p_{1 .}+p_{.1}\right)+p_{1 .}-p_{1 .}^{2}+p_{.1}-p_{.1}^{2}\right] \\
& =n^{2} p_{1 .} p_{.1}\left[1+p_{1 .}^{2}+p_{.1}^{2}+2 p_{1 .} p_{.1}-p_{1 .}-p_{1 .}^{2}-p_{.1}-p_{.1}^{2}\right] \\
& =n^{2} p_{1 .} p_{.1}\left[1+2 p_{1 .} p_{.1}-p_{1 .}-p_{.1}\right]
\end{aligned}
$$


To complete the derivation we will substitute these expressions back into the original and the asymptotic variance of $\sqrt{n}[g(\hat{\mathbf{p}})-g(\mathbf{p})]$ is

$$
\begin{aligned}
& n^{2}\left[\sum_{i, j=1}^{2} p_{i j} \phi_{i j}^{2}-\left(\sum_{i, j=1}^{2} p_{i j} \phi_{i j}\right)^{2}\right]=n^{2} p_{1 .} p_{.1}\left[1+2 p_{1 .} p_{.1}-p_{1 .}-p_{.1}-p_{1 .} p_{.1}\right] \\
& =n^{2} p_{1 .} p_{.1}\left[1+p_{1 .} p_{.1}-p_{1 .}-p_{.1}\right]=n^{2} p_{1 .} p_{.1}\left(1-p_{1 .}\right)\left(1-p_{.1}\right) . \text { Thus, the asymptotic }
\end{aligned}
$$

variance of $n_{11}-E_{11}$ is $\frac{n^{2} p_{1 .} p_{.1}\left(1-p_{1 .}\right)\left(1-p_{.1}\right)}{n}=n p_{1 .} p_{.1}\left(1-p_{1 .}\right)\left(1-p_{.1}\right)$ which can be estimated by $E_{11}\left(1-\hat{p}_{1 .}\right)\left(1-\hat{p}_{.1}\right)$. Finally, this result can be extended to any cell in the $I x J$ table (1.3.1) if we simply consider that the row 2 and column 2 in our derivation represent the sum of all other cells in the larger contingency table. Therefore, $\operatorname{Var}\left(n_{i j}-E_{i j}\right)=E_{i j}\left(1-p_{i}\right)\left(1-p_{. j}\right)$ and the formula for the adjusted Pearson residual is

$$
r_{i j}=\frac{\left|n_{i j}-E_{i j}\right|}{\sqrt{E_{i j}\left(1-p_{i .}\right)\left(1-p_{. j}\right)}}
$$

Since $p_{i j}$ is asymptotically normal, so is $r_{i j}$.

As stated above this method has not yet been used in this field. In the data example in Chapter 5 and the simulation study in Chapter 6, this method will be put to the test. Since $r_{i j}$ has an asymptotic standard normal distribution this statistic can be directly compared with critical values taken from a Z-score table to assess a suspiciously large interaction between drug $D_{i}$ and ADR $A_{j}$. 


\section{Chapter 5}

\section{Data Example}

The topic of this discussion has been on methods of signal detection in large dimensional contingency tables, but more specifically it is on the search for potentially dangerous drugs. This applied aspect is commonly addressed using simulation studies as will be discussed in the next chapter, however another method that will allow us to observe the methods in action is using real data. The difficulty in analyzing real data is defining a so called 'true' signal for which to search. In the last decade or so pharmacologists have flagged a number of side effects of drugs that we can consider to be fairly certain, but the nature of science is that nothing is ever true, only hypothesized to be true. As such choosing the appropriate set of drugs and ADRs to create a real life data set requires some consideration.

In 1999 Vioxx, a non steroidal anti inflammatory drug (NSAID), was approved by the FDA and released onto the U.S. market. In 2000, a study known as V.I.G.O.R. (Vioxx G.I. Outcomes Research) (Bombardier et al., 2000) designed to monitor stomach ulcers and bleeding as a side effect of Vioxx, reported an increase in cardiovascular risk. Another study known as APPROVe (Adenomatous Polyp Prevention on Vioxx) (FDA website), that was designed to investigate the effect Vioxx had on prevention of colon polyps, also reported similar findings. Due to these and a number of other studies, Merck recalled Vioxx and removed it from the market in September 2004. Vioxx is a drug that 
exhibits large enough correlations with certain types of ADRs that it is worth examining the impact in SRS data during that time frame. The data comes directly from the FDA's Center for Drug Evaluation and Research (CDER) website. This database of information was updated quarterly. Due to the Vioxx recall date of September 2004, we will examine the second quarter of that year, just a few months prior to the removal of Vioxx from the marketplace.

Another important consideration is what drugs and ADRs should make up the reference set. From working with data in simulation studies and from our discussion on the methods, a few important considerations have come to light. First of all, sparseness of the data matrix will make the estimators perform poorly, especially when the number of nonzero cells in a row or column is extremely low. Thus, to deal with this concern, picking fairly common ADRs with respect to the drugs in question will alleviate this problem. The second issue is whether to pick drugs and ADRs that are similar in nature or drugs and ADRs that are completely different. However, since the purpose of this example is to simply demonstrate the estimators in action, we will approach this as simplistically as possible. Thus, we will choose a set of drugs that are all NSAIDS and therefore have a significance presence in the data set. Thus, the NSAID reference set is comprised of Celebrex, Mobic, Athrotec, Daypro, Motrin, Naprosyn, Volaren, Indocin and Bextra. For the set of ADRs, we want to observe ADRs with large counts with respect to Vioxx. After sorting these ADRs it becomes evident that the largest observed counts are associated with pain-related ADRs. This is to be expected since the purpose of 
taking Vioxx is to alleviate pain related symptoms. It is important to mention that although this may be a concern under some circumstances, we are simply using the information in these cells to generate a background frequency to compare against the 'true' signal. Also we are not attempting to make inference on Vioxx itself, this is simply to demonstrate the methods in practice. Thus, the ADRs we will use are pain, hypertension, back pain, arthritis, osteoarthritis, arthralgia, rheumatoid arthritis, diabetes mellitus, depression and prophylaxis.

The next step to this demonstration is the decision of which pair in our data set is deemed a 'true' signal. Here it is important to avoid classifying the pain-related ADRs as signals since they may appear heightened due to the fact that they are more than likely the symptom causing people to take NSAIDs in the first place. Also note that the fact that the drugs in the reference set are all NSAIDs should lessen the impact of the pain-related ADRs since there should be a correlation between them and all the reference drugs. One of the major concerns with Vioxx was its effect on the cardiovascular system. A number of studies have been conducted on this topic and the results have demonstrated that this group of ADRs does have some correlation with the drug. For our example we will focus on a precursor to cardiovascular events, hypertension. A number of studies (Whelton et al., 2002; Solomon et al., 2004) have shown increased levels of blood pressure in patients taking Vioxx versus patients on other NSAIDs as well as non NSAIDs.

To conduct the analysis, the data matrix, figure (1.3.1), is run through the methods of signal detection at a significance level of $5 \%$. At each cell, the remainder of the matrix 
is collapsed into the $2 \times 2$ table (1.3.2) and the methods are run using this condensed table. Then we move to the next cell and the procedure is repeated. The resulting matrices contain only ones and zeros, where the ones represent that a signal is flagged. Note that the empirical Bayes procedure requires a maximization of the likelihood function and as such the possibility of divergence in the maximization routine exists. Under this circumstance the corresponding method is skipped and a signal is not recorded. Also for the Chi Square Yates, it is important that the expectation of the individual cells is greater than five to satisfy the assumptions. If this is not reached, once again a cell may be skipped. It is important to recognize that the only assumption we are making here is that the previously named pair is a 'true' ADR and we are searching for that specific signal. However, even that assumption may be a stretch and, as such this data example is limited to the accuracy of that statement.

\begin{tabular}{|c|c|c|c|c|c|c|c|c|c|c|}
\hline & PAIN & HYPERTENSION & $\begin{array}{l}\text { BACK } \\
\text { PAIN }\end{array}$ & ARTHRITIS & OSTEOARTHRITIS & ARTHRALGIA & $\begin{array}{l}\text { RHEUMATOID } \\
\text { ARTHRITIS }\end{array}$ & $\begin{array}{l}\text { DIABETES } \\
\text { MELLITUS }\end{array}$ & DEPRESSION & PROPHYLAXIS \\
\hline vioxx & 854 & 809 & 624 & 568 & 566 & 550 & 324 & 199 & 188 & 175 \\
\hline CELEBREX & 145 & 44 & 53 & 81 & 63 & 50 & 127 & 17 & 1s & 11 \\
\hline MOBIC & 8 & 16 & 8 & 13 & 8 & 6 & 78 & 5 & 3 & 2 \\
\hline ARTHROTEC & 12 & 0 & 0 & 1 & 0 & 10 & 34 & 0 & 2 & 4 \\
\hline DAYPRO & 30 & 20 & 5 & 5 & 0 & 1 & 4 & 0 & 6 & 5 \\
\hline MOTRIN & 104 & 49 & 18 & 26 & 2 & 18 & 17 & 2 & 10 & 15 \\
\hline NAPROSYN & 6 & 5 & 1 & 0 & 11 & 0 & 13 & 2 & 3 & 1 \\
\hline VOLTAREN & 20 & 19 & 15 & 2 & 26 & 19 & 118 & 10 & 11 & 14 \\
\hline INDOCIN & 1 & 12 & 8 & 7 & 9 & 0 & 26 & 1 & 1 & 0 \\
\hline BEXTRA & 22 & 11 & 18 & 70 & 44 & 30 & 39 & 2 & 13 & 1 \\
\hline
\end{tabular}




\subsection{Results}

The output of this algorithm is multiple result matrices, where a one represents a signal flagged and a zero represents a lack of a signal. To summarize the results, we will focus on the predefined signal pair, Vioxx/Hypertension, but keep in mind that the actual resulting matrices are given in Appendix 1. Below is a table representing the decision made by each method and whether or not it is a correct decision by our criteria. Note that we examined twelve methods, and of these, a third were unable to detect the signal. Specifically, the PRR cutoff method, the sequential probability ratio test and both the WHO methods were unsuccessful

\begin{tabular}{|c|c|}
\hline & Decision \\
\hline Yates & TRUE \\
\hline adj.resid & TRUE \\
\hline EB.dist & TRUE \\
\hline EB.roux & TRUE \\
\hline PRR.cutoff & FALSE \\
\hline ROR & TRUE \\
\hline$Q$ & TRUE \\
\hline SPRT & FALSE \\
\hline WHO.approx & FALSE \\
\hline WHO.exact & FALSE \\
\hline
\end{tabular}

However, we cannot make any conclusions on the capability of these methods based on this exercise alone. Note that the PRR cutoff requires a value greater than two; however recall that the PRR compares the relative frequency of Hypertension as an ADR found in association with Vioxx against the relative frequency of Hypertension found in all other drugs. However the fact that our reference set are all NSAIDs implies that the 
relative frequencies could very plausibly be quite similar since it is quite possible that Hypertension is an ADR for all of these drugs; however it may not be as strong. As such a PRR of two may be too high as a cutoff versus a confidence interval for the PRR which has a better ability to not be influenced by this possible issue. As far as the SPRT goes, the statistic in question is set up to detect signals based on the assumption made by Roux et al. (Roux et al., 2005); that is that a signal is defined as two times the relative risk of a non signal. This use of relative risk in the definition gives us similar issues as with the PRR. If the data set is such that the relative frequencies are not significantly different, a signal may escape the SPRT. Finally, the WHO methods are applied as neural networks since the updating of the prior distributions tightens the bounds on the confidence intervals and causes the estimates of the information component to be more and more realistic. However, in this example we are not allowing this process to occur since we are simply looking at a particular moment in time. Thus, it seems reasonable for the WHO method to not necessarily flag a signal.

It is worth noting that this exercise was conducted for the purpose of demonstrating the use of the methods and not to make any inference on Vioxx's effect on Hypertension, or to judge the performance of these estimators. To deal with these questions, we will turn to a simulation study to gain more insight into how well these estimators perform, and under what conditions they perform best. 


\section{Chapter 6}

\section{Simulation Study}

\subsection{General Concerns}

Now that we have established some of the mathematical properties of the estimators and have taken a look at the methods applied to real data, another important consideration is how they will perform in the broader sense. As noted in the data example, this is not an easy thing to examine. The first major issue, which was previously discussed, comes from the fact that we are attempting to make recommendations about possible correlations as opposed to testing a population for some known condition. These correlations are not representations of an interaction that we can verify as the 'truth', simply because in medical sciences very few things can be applied across all patients. For example, if we find a correlation between smoking and lung cancer we cannot say that all smokers will get lung cancer. Thus, how do we truly test our methods in practice?

A common method in statistics is to use simulation studies where the "truth" is known. Unfortunately, we run into difficulty based on the limitation of how to define a curiously large interaction between an ADR/drug pair. Since the knowledge of this relationship is minimal, we can only assume certain things about these variables and the 
way they influence each other. We will start by attempting to assign a probability distribution to the random variable $n_{i j}$.

In the discussion on the PRR and ROR, the cell counts were treated as binomially or multimonially distributed random variables. Generating random data from these distributions requires that we consider the problem as one of sorting, in which we are attempting to categorize observations into specific groups. However, in order to accomplish this we need an idea of the probability of an occurrence. Since the probability of witnessing an interaction between a specific drug and ADR changes from drug to drug and ADR to ADR, it is extremely difficult to attempt to estimate these probabilities. Another choice that is commonly considered in pharmacovigilance is to assume a Poisson distribution to define the behaviour of the random counting variable $n_{i j}$. This intuitively makes sense because the Poisson distribution is an integer-based counting distribution that is used to count rare events. Due to the fact that we are working with a contingency table, each drug and ADR is compared to all other drugs and ADRs, yielding an extremely sparse data matrix, which can thus be interpreted as rare events. The additive property of the Poisson also implies that all cells in our condensed matrix are accounted for in distribution. Therefore, a Poisson distribution seems like a reasonable choice.

Now that we have a distribution in mind, the difficulty lies in choosing the parameter of the Poisson distribution. A common view (Tubert and Begaud, 1991; Roux 
et al., 2005) is that the rate should represent a scaled value of relative risk, such that the relative risk is the deciding factor on generating a signal, $\lambda=R R^{*} I^{*} T^{*} p_{i j}$ where $I$ represents background incidence rate, $T$ represents exposure rate to a given drug and $p_{i j}$ is a measure of some sort of reporting probability. Again all of these parts are extremely variable in real life, and as such are hard to interpret. For instance, we have an idea of background rates for ADRs that cause severe problems, however for ones that are mild in nature, such as a headache, we have no idea of the 'true' background rate. Even if we are able to poll $100 \%$ of the population as to their headache patterns, the amount of bias that would exist would cause the rates to be off. As far as exposure to the drug is concerned, we can hypothetically gather that sort of information through patient files. However, we are limited to prescription drugs, and as such, the estimates will again be limited. Finally, reporting probability is defined as the probability that an individual will choose to report an ADR they are experiencing. This probability is even harder to estimate because of the massive amounts of bias associated with this subjective measure. To begin, the severity of the ADR will alter this probability as well as the perception of the rarity of the ADR. Variation among personality types will cause different rates, as well as practical issues, such as knowledge of where to report, among others.

Roux et al (Roux et al., 2003) attempted come up with a method of better estimating some of these values. They did this by creating a set of fuzzy coding to condense a number of situations into an algorithm for the purposes of simulation. To begin, each of the previously listed variables making up $\lambda$ are considered categorical. Exposure to the 
drug $\left(T_{i}\right)$ can take on values of 'launch', 'growth', 'maturity', 'decline' and 'end of life'. Each of these categories is set to a specific value of $T_{i}$ which is a function of $T_{i \max }$, or the maximal drug exposure. The distribution of $T_{i}$ is skewed right, which implies that the consumption of the drug will increase rapidly in the early stages after release onto the market and slowly taper off over time.

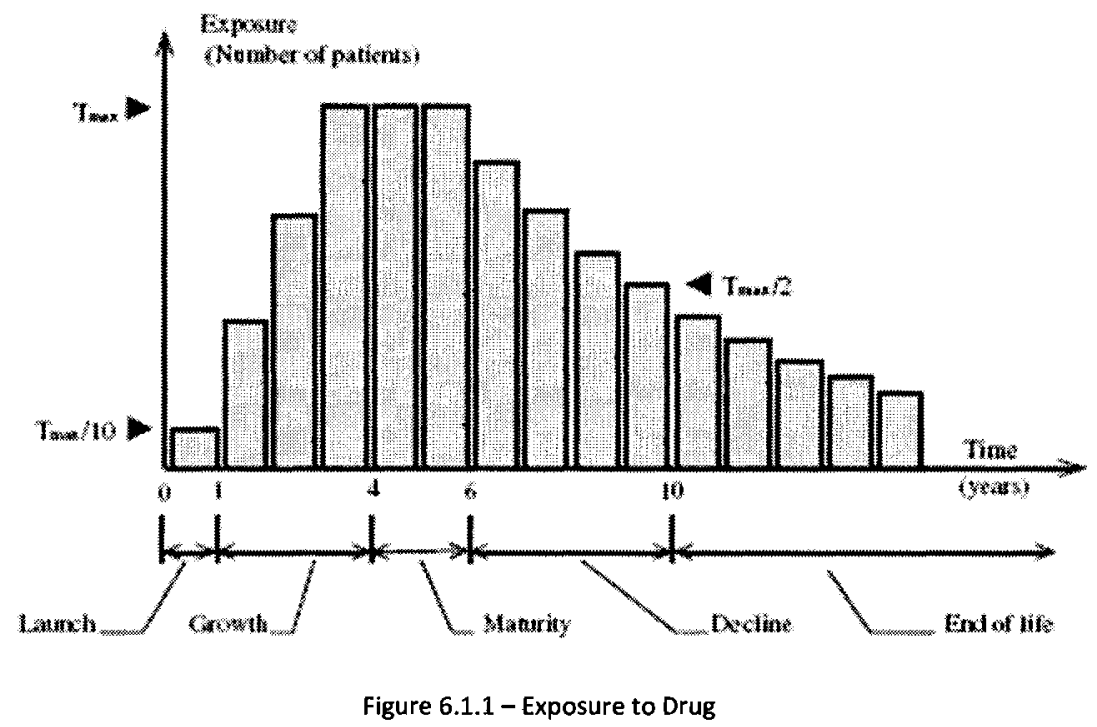

In figure 6.1.1 it is evident that each cycle in the life of the drug has a particular relationship with $T_{i \max }$, and it seems that Roux chose two particular values (other than $\left.T_{i \max }\right)$; specifically $T_{i \max } / 10$ was selected for the launch period and $T_{i \max } / 2$ for the beginning of the decline phase.

To tackle the complex problem of reporting probability $\left(p_{i j}\right)$, Roux et al recognized that there are three situations that influence this value (Tubert-Bitter et al, 
1998): the more serious the event, the more frequently it is reported, the more unknown the relationship between the drug and the ADR the more reported and finally, the more recent the drug was released the more the ADR is reported. They also restrict the range of this probability from 0 to 0.1 . Based on the preceding three notions, Roux et al assert that a 'membership' function can be derived. Specifically, this is a function that specifies the category in which $n_{i j}$ resides, and it is obtained by first comparing the cumulative number of reports with the delay since drug launch. The variable, cumulative number of reports is coded as 'low', 'medium' or 'high' which corresponds to [0,5], [0,20], and [5, $\infty$ ] respectively. The delay since drug launch corresponds to the discussion of the previous paragraph. This comparison is made with the following chart. The output gives us a category for reporting probability.

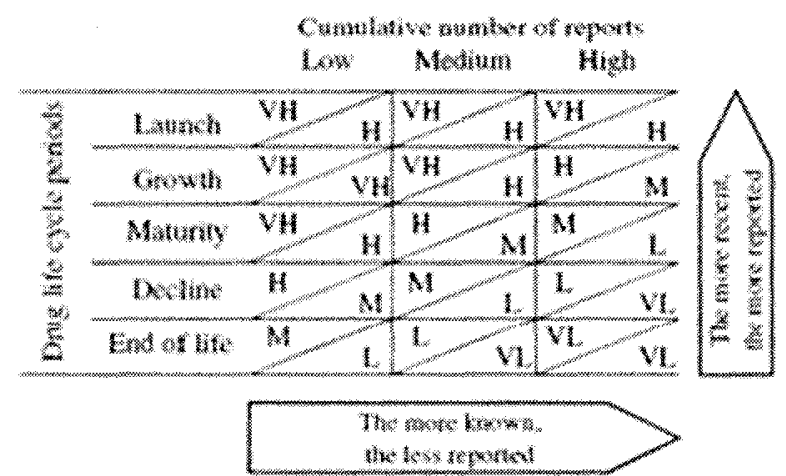

Figure 6.1.2 - Reporting Probability

Note that each cell in figure 6.1.2 has two values for $p_{i j}$. These correspond with the seriousness of the ADR, where the upper value is considered 'serious' and the lower value is 'mild'. 
The next step is to create the required membership functions. The three categories for which we have associated with $n_{i j}$ now take on particular values to compare with membership values.

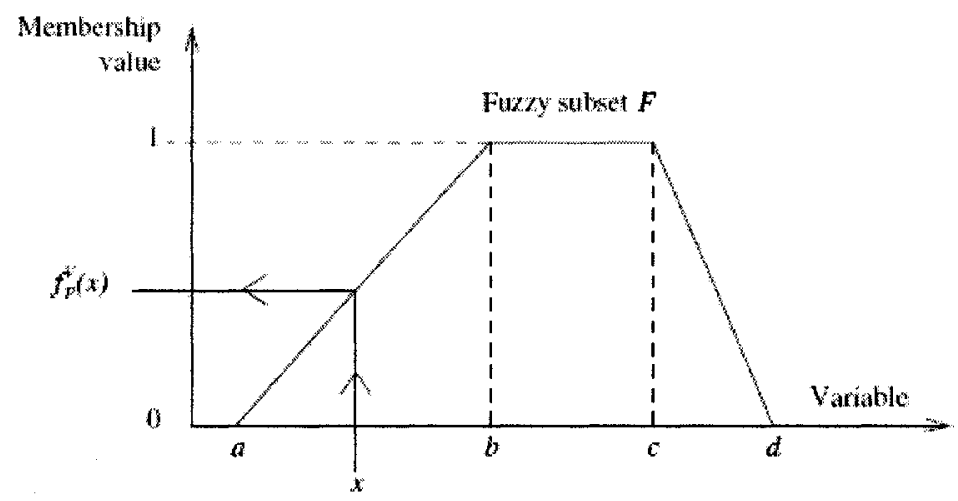

Figure 6.1.3 - Membership Function

\begin{tabular}{|c|c|c|c|c|c|}
\hline Vortalda & 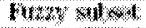 & $a$ & 6 & 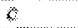 & 1 \\
\hline \multirow{3}{*}{$\begin{array}{l}\text { Cwmothrs } \\
\text { repont mont }\end{array}$} & Wor & 0 & t) & 0 & 8 \\
\hline & 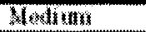 & 0 & 8 & 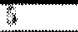 & 20 \\
\hline & HXV & 5 & 30 & $4 \%$ & $4 \mathrm{x}$ \\
\hline \multirow{5}{*}{ 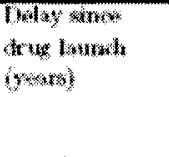 } & 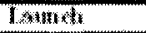 & (I) & is & 0 & 1 \\
\hline & 8 insolh & \% & 1 & 1 & 4 \\
\hline & Tatomky & $i$ & 1 & 3 & 10 \\
\hline & $\mathrm{T} \times \mathrm{hlw}$ & 6 & 70 & 10 & W \\
\hline & End ghe & 10 & 30 & 18 & $4 \%$ \\
\hline \multirow{5}{*}{ 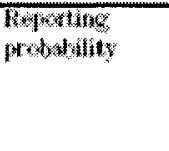 } & Wo W W & 0 & $\theta$ & 0 & 002 \\
\hline & The & 3 & 0012 & TOI2 & 002 \\
\hline & Medrum & 0012 & 002 & 062 & 60 \\
\hline & Whol & hetas & $\pi n$ & Wh & 71 \\
\hline & Vhr Hgls & 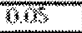 & NI & OI & 01 \\
\hline
\end{tabular}

Figure 6.1.4 Values associated with the Membership Function

Figure 6.1.4 shows us the values that make up these membership functions, while figure 6.1.3 gives us an idea of what the function looks like. Note that if $b=c$, then the membership becomes a triangle as seen when the delay since drug launch is in the growth 
phase. The function characterizing this graphical display is $f_{F}^{V}(x)=\max \left(\min \left(\frac{x-a}{b-a}, 1, \frac{d-x}{d-c}\right), 0\right)$ where $V$ is the variable being 'fuzzified'.

Finally to reach a value of $p_{i j}$, we must specify a conclusion. This conclusion is reached by comparing the minimum of the membership values for the cumulative number of reports and delay since drug launch to the value of the membership function for $p_{i j}$. Taking the minimum gives us our conclusion. Roux et al gives an example where we are assuming that the event is mild, the cumulative number of reports is low and the delay since drug launch is in the launch period. This specifies a value of high for $p_{i j}$ and we get the resulting conclusion function:

$$
C_{R}\left(N_{o b s}, D_{o b s}\right)=\min _{x \in[0.025,0.1]}\left(f_{\text {High }}^{p_{i j}}(x), \min \left(f_{\text {low }}^{N}\left(N_{o b s}\right), f_{\text {Launch }}^{\text {delay }}\left(D_{o b s}\right)\right)\right)
$$

where $N_{o b s}$ is the total number of reports, $D_{o b s}$ is the delay since drug launch and $R$ is the rule listed above. The corresponding membership functions are as follows

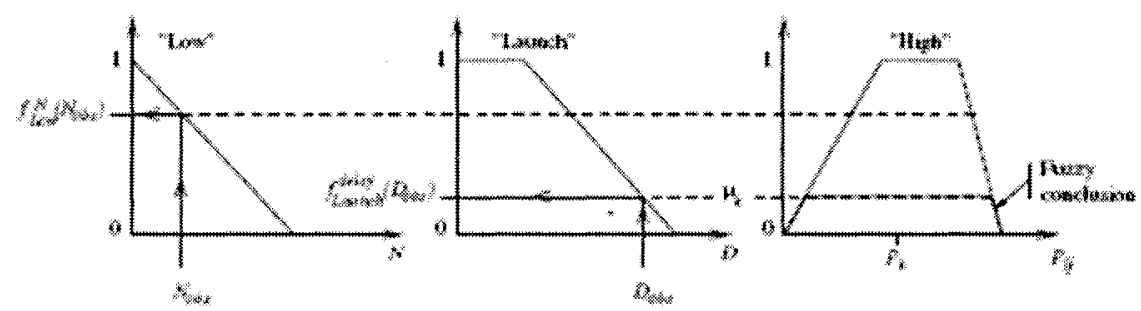

Figure 6.1.5 - Membership Functions used in example 
Finally Roux proposes a 'defuzzification' process known as the Height Method (Eklund et $a l ., 2000$ ). This process gives us our final value for $p_{i j}$ which is calculated as $p_{i j}=\frac{\sum_{k} \mu_{k} p_{k}}{\sum_{k} \mu_{k}}$. As can be seen in figure $6.1 .5, p_{k}$ represents the mean of the maximum of the membership function associated with the $k^{\text {th }}$ subset of $p_{i j}$ and $\mu_{k}$ is effectively the conclusion of the $k^{\text {th }}$ subset of $p_{i j}$.

Although Roux et al have improved the approximation of the reporting probability, the Poisson rate $\lambda$ is still just a scaled relative risk. Therefore, it seems as though the signal detection methods used in simulation are really attempting to find a heightened value of relative risk given the level of scaling discussed above. Until we have a better method of flagging signals in simulations, they will be limited to searching for this outcome. In the meantime, this seems reasonable since relative risk is comparing the probabilities of observing a specific ADR/drug combination compared to the same ADR across all other drugs. However, using relative risk assumes that the data matrix is complete and contains all possible drugs. Since this is not the case, in practice if a set of drugs is excluded, the relative risk of the actual data will be altered. Therefore signals can actually be masked by incomplete data and the resulting lowering of the relative risk. That being said, the current method of simulation gives us a partial picture of the performance of these estimators. 


\subsection{Methods}

The simulation study conducted here is based on similar reasoning as previously discussed. The random variable $n_{i j}$ is considered as a Poisson distributed variable with an unknown mean. Before discussing the determination of this mean, we will first look at some of the necessary variable definitions. To begin, the simulation was run using the $\mathrm{R}$ software version 2.7.1. The data sets are matrices with dimensions 12 rows by 15 columns. The rows are considered to be drugs and the columns ADRs; they are named 'N.drug' and 'N.event' respectively. Note that these input parameters can be altered by the user throughout the code. A random seed is specified so that the results can be reproduced. Specifically, the variable ' $z$ ' is a marker that keeps track of the seed value. In addition, the variable 'num.run' which represents how many runs have occurred. Also the number of runs per given set of parameters is fixed at 1000 here, however once again the user can alter this by adjusting the variable 'run'.

As stated previously each element of the data matrix is a random Poisson variable with an unknown mean. In this study we consider the mean to be a scaled relative risk, similar to our discussion on Roux's methods. However, since this scaling is unknown and we are effectively examining how well these estimators detect heightened levels of relative risk, the weights are generated as simply as possible. Uniform random variables are used as well as fixed sequences of numbers in order to create weights that will be variable enough so as to not give similar weights each time, but simple enough to minimize the impact of a biasing underlying process on the data set. However, a major 
concern of this process is the potential issue of sampling variability created by the random generators and the effect that is observed in the outputted matrix. Thus it is important to consider what parameters to give to the random generators throughout this entire process and to consider the potential tails of resulting distributions for unusual behaviour.

Another concern when picking these parameters is the general sparseness of the data matrix. If a Poisson random number generator has a rate of approximately zero, the probability of not generating a zero is extremely low. For example if the rate was $\lambda=0.01$, then $P\left(n_{i j}>0\right)=1-P\left(n_{i j}=0\right)=1-e^{-0.01}=0.0099$. This is a concern due to the fact that our estimators are dependent on the surrounding data. We can assume that each row and column needs to have a minimum of one nonzero cell. Otherwise that drug or ADR would have never been entered into our data set. Thus, the rate needs to be large enough to ensure this assumption is satisfied. If not, we run the risk of division-by-zero errors in estimators such as the PRR or ROR. The other major issue is the maximization procedure in the empirical Bayes method. Since this method is a nonlinear maximization, convergence rates will not be perfect; however an extremely sparse data matrix will lower those rates. Thus we ideally want a rate that will create a data set that is sufficiently dense to avoid any problems.

Now that we have discussed some of the dangers to be aware of, we will look at the individual components that Roux et al consider in their simulation. The first issue 
that arises given the concerns mentioned is the range of $p_{i j}$. The general consensus is that $p_{i j}$ ranges from 0 to 0.1 (Tubert et al., 1991) as can be seen in the fuzzy coding description above, however this would allow for extremely small values. In other words, as $p_{i j} \rightarrow 0$, the Poisson rate $\lambda \rightarrow 0$ and begins to start producing extremely sparse data sets. In fact, even if the range was condensed to 0.01 to 0.1 , we will still encounter difficulties. An order of magnitude can be the difference between an overly sparse data set and a data set that causes no problems. For instance, if we consider the probability of not generating a zero with a Poisson rate of 0.1 , the resulting probability will be 0.099 , or one order of magnitude larger than the previous example with a rate of 0.01 . To avoid issues arising from a sparse data set, and given the reasons previously stated, the value of $p_{i j}$ will be incorporated into another random variable as a fixed value, namely the background incidence.

When dealing with background incidence, the considerations of practicality are once again set aside. It is impossible to come up with reasonable values of background incidence for this simulation study, largely due to the fact that we are not simulating a particular set of drugs. Thus a random uniform number generator is used for this quantity. The parameters are specified as ranging from 0.002 to 0.007 . Incorporating $p_{i j}$ at 0.1 into these parameters, we arrive at $I \sim U$ nif $(0.0002,0.0007)$. The other value being considered is the exposure to drug, or $T$. This value will be fairly large as its unit of measurement is the number of people. Thus, a range of values starting at 200,000, and 
increasing incrementally in steps of 10,000 is used. Since our desired outcome is a matrix with dimensions 'number of drugs' by 'number of ADRs', we will generate two vectors. The vector $I$ will be of length 'number of ADRs' and $T$ will be of length 'number of drugs'. Finally computing $I T^{T}$ will give us our data matrix. Note that these variables are identified as 'expo' for drug exposure and 'backmatrix' for the background incidence.

The last component is to determine how to flag certain cells as signals. Once again, this is done using a heightened value of relative risk. In this simulation, there are 15 drugs and 12 ADRs for each data set and of $10 \%$ of the cells ( 18 cells) are considered signals. To select which cells are signals, a random uniform generator is used with parameters 1 and 180 . The values are then rounded to get integer values corresponding to positions in the matrix. Note that the $\mathrm{R}$ software will consider a single cell value to correspond with the position in the matrix if we were to consider the matrix to be one large vector of the columns of the matrix. The user will specify the relative risk as the variable ' $R R$ '; a fixed value is required. This value should be picked carefully as three random variables are created using this input. Since our ultimate goal is to examine how the estimators perform in practice, it seems reasonable to investigate their performance under different distributions. Hence, we have applied three conditions for the generation of signal size. The basic value, ' $R R f$ is simply a fixed user-inputted value, 'RRe' is generated from an exponential distribution and ' $R R u$ ' is generated from a uniform. To attempt to create some similarity between the values of relative risk, we will first keep the 
values of the mean of the uniform and the exponential the same as the user-inputted relative risk. Also we will determine the parameters of the uniform, keeping the coefficient of variation the same between the exponential and the uniform. In other words, the rate of the exponential will be $1 / R R$ and the parameters of the uniform are determined by solving the simultaneous equations, $\frac{a+b}{2}=R R f$ and $\frac{b-a}{\sqrt{12}}=R R f$. These equations are derived by combining the fact that the coefficient of variation for the exponential distribution is one coupled with the fixing of the mean of the uniform to be the same as the fixed value of relative risk. We can then solve for $a$ and $b$ as functions of $R R f$, yielding $a=2 R R f-b$ and $b=\frac{R R f(\sqrt{12}+2)}{2}$. All this is calculated in the function 'RelRisk()' in the $\mathrm{R}$ software. Upon completion the values of relative risk are applied to the corresponding data matrices.

Now that we have the method for simulating pharmacovigilance data sets, it is important to consider some of the potential issues regarding simulation. The major problem encountered in the methods is the maximization in the empirical Bayes method. The algorithm requires a non-linear maximization scheme such as the Newton Raphson that was discussed in section 3.2.2. Using R's 'nlminb()' procedure, we can generate the values for our parameters keeping in mind that a number of the values obtained will lead to divergence. Since the distribution of the mixture of negative binomials will be changing continuously due to changing parameters, it seems only natural that divergence cannot be avoided. A method for dealing with this issue is to adjust the starting point for 
the parameter search. Thus, prior to each simulation, a "pilot simulation study" of 100 runs was conducted for the purposes of getting estimates of how many runs would diverge in the main simulation. This was done by using the code for generation of the data set and then running $n \operatorname{lmin} b()$ on each data set generated. A counter was used to keep track of the number of divergences in 100 runs. If this proportion exceeded $25 \%$, different starting values were attempted. When the ideal proportion was achieved, those values were incorporated into the larger simulation. Finally a counter was used in the larger simulation to keep track of the number of divergences to ensure that the estimates from the pilot simulation were fairly accurate.

Now that we have considered some of the potential issues, we will examine the algorithm. To start, we have eleven methods of assessing a signal as follows:

\begin{tabular}{|l|l|l|l|}
\hline 1 & if $P R R \geq 2, \chi_{Y}^{2} \geq 4, n_{i j} \geq 3$ & 7 & if the lower bound of $I C>1$ (approx) \\
\hline 2 & if the lower bound of $P R R>1$ & 8 & if the lower bound of $I C>1$ (exact) \\
\hline 3 & if the lower bound of $R O R>1$ & 9 & $\begin{array}{l}\text { direct comparison of observed to } \\
\text { quantiles of the posterior distribution }\end{array}$ \\
\hline 4 & if the lower bound of $Q>1$ & 10 & if $\mathrm{EBp}>0.5$ \\
\hline 5 & If $\chi_{Y}^{2}>3.84$ & 11 & if $r_{i j}>1.96$ \\
\hline 6 & $\ln (2) \cdot n_{i j}-E \geq 2.94$ & & \multicolumn{2}{|c|}{} \\
\hline
\end{tabular}

Each of these methods will flag a potential signal in our matrices of results. Note that due to the disproof of the variance of the logarithmic posterior in the empirical Bayes 
method, the confidence interval is left out of analysis. Each of the methods above will be used at three different levels of significance where possible, namely $10 \%, 5 \%$ and $1 \%$. There are a number of matrices of results that come out of this algorithm where each cell is a count of how many times the method flagged a signal for that individual cell. The algorithm is as follows:

1) Generate data set

2) Conduct the nonlinear maximization for the empirical Bayes method if this diverges set an indicator variable to skip the empirical Bayes method.

3) Initialize the results matrices. They are of the same dimension as the data matrix.

4) Go to cell $i, j$ in data matrix and condense the remaining data set into a $2 \times 2$ table (1.3.2)

5) For that individual cell run all the methods and add one to the corresponding cell in the corresponding results matrix.

6) Repeat 4 and 5 for all $i, j$.

7) Repeat 1-6 1000 times.

Finally, we are left with the matrices of results that enable us to calculate the proportion of signals flagged at each simulated ADR/drug combination, which will allow for analysis and comparison. 


\subsection{Results}

To analyze these matrices of results it must first be noted that we have a large number of them, and for simplicity's sake we will only review a representative group of them. To start out we have a number of different comparisons available to us. First, we will take a look at a comparison of the estimators against each other by examining the data created with a fixed value of relative risk, or signal size. After we get an idea of how the estimators compare with each other we can begin to examine some of the other issues such as changing the significance level and changing the underlying distribution generating the signal size. Another problem that will be discussed is whether the Type one error rates match up with the specified significance levels, and the impact of this on power. Also, note that the detection method proposed by Roux et al (Roux et al., 2005) does not prespecify an alpha level and as such will be examined more thoroughly on its own.

To examine the performance of the estimators we will conduct a power analysis of the results and compare scatter plots generated from the data. To begin, recall our hypothesis in section 2.1 stated in the introduction to Chapter 2 , and note that our null hypothesis is one of no association between drug and ADR. The matrices of results are such that each cell represents the number of signals flagged. Since we know which cells are predetermined signals we can calculate the power by simply dividing the number of signals observed in the "signal" cells by 1000 . These proportions are estimates of the probabilities of deciding a signal exists when in fact there is an association between drug 
and ADR. The other calculation we can make is to verify that the Type one error rates are close to the prespecified significance level. This is done by noting that the other cells are naturally not signals and thus the counts in these cells represent a rejection of the null hypothesis when it is in fact true. By dividing the number of observed signals in these cells by 1000 yields a proportion that represents an estimate of the Type one error rate for the given cell. Finally, note that the denominator of this proportion is ideally 1000 , however some of the methods may have lower values. The empirical Bayes methods require a maximization routine that will not necessarily converge, and in the event of divergence a particular run will be skipped and the denominator must be adjusted accordingly. Fortunately, this number is counted in the algorithm under 'diverge.count' and this adjustment is easily implemented. This will allow us to adjust the denominator accordingly and we are now in a position to take a look at the values of Power and Type one error.

For comparative purposes we will graph the values of power versus relative risk. The value of relative risk is the strength of signal and values less than or equal to one represent no signal. To aid in the graphical representation we will employ a non parametric smoothing routine known as Lowess or locally weighted least squares. This mechanism, originally established in 1979 by W.S. Cleveland (Cleveland, 1979), fits local regression functions to a neighbourhood of points based on a proportion of points specified by the user, $q$. The Euclidean distance is calculated for the farthest point from the point in question, $d_{q}$, and is applied to a weight function as follows, 


$$
w_{i}= \begin{cases}{\left[1-\left(d_{i} / d_{q}\right)^{3}\right]^{3}} & d_{i}<d_{q} \\ 0 & d_{i} \geq d_{q}\end{cases}
$$

In other words if the point is outside of the neighbourhood, it receives a weight of zero, and the rest receive weights that will decrease with respect to the Euclidean distance from the point in question. A weighted least squares estimate is obtained at each point based on the weights determined by (6.3.1), and a response surface (function) is gradually created. The resulting points are a smoothed function that has the advantage of not requiring any assumptions of the underlying response function. In our application, this algorithm is sufficient for producing power curves for graphical comparison. 


\subsection{Comparison of methods}

To begin we will consider the differences in the signal detection abilities of the individual methods by using the data with a fixed signal size and plotting the power curves of the methods on a single plot for the three significance levels. We will examine an alpha of $5 \%$ and will only look at the Lowess curve since the scatterplot is fairly messy and difficult to read.

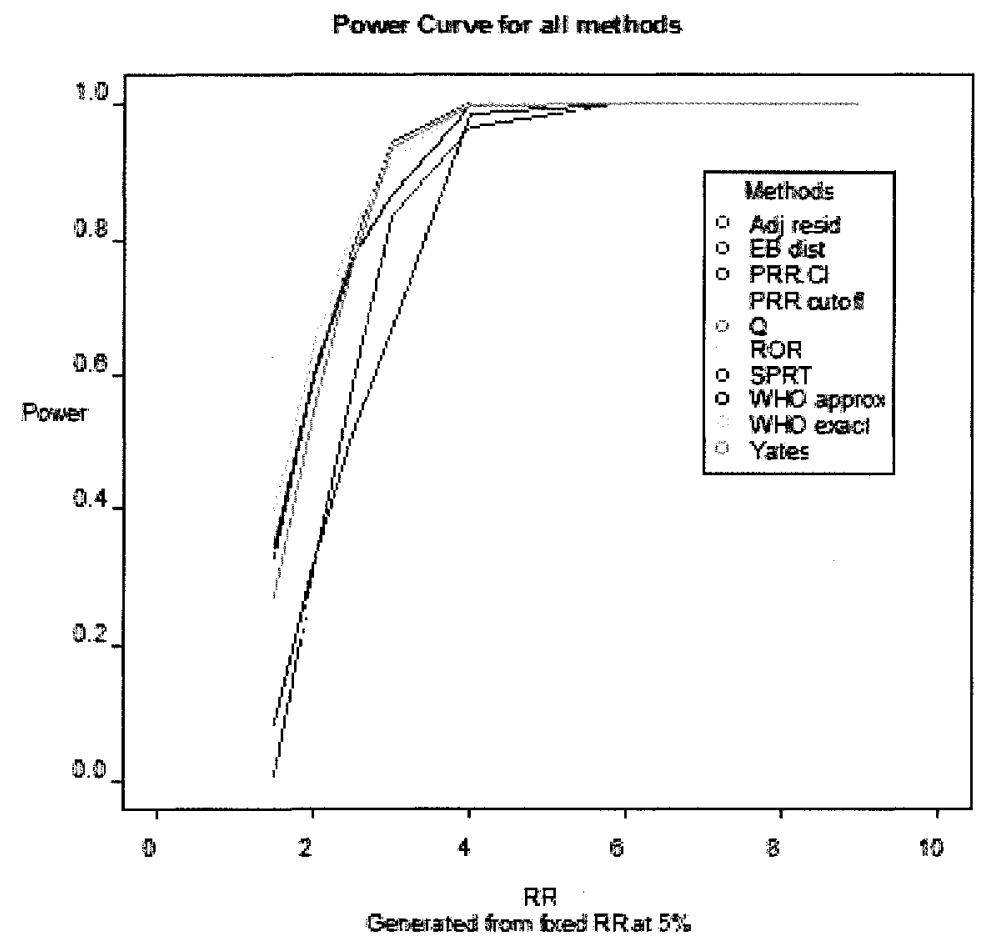

Figure 6.4.1 - Power versus RR for all methods at a 5\% significance level

It seems that at a relative risk of 4 , all the methods do fairly well. However between 1.5 and 4, there is a fairly large difference in the methods. The ROR, Yule's Q, PRR confidence interval and the Chi-square Yates seem to perform the best with the 
exception of the WHO exact method which out-performs these between 1.5 and approximately 2 , but then drops off after that. The WHO approximation method has lower power then all the previously-mentioned methods in this range. The SPRT, empirical Bayes and PRR cutoff method all seem to perform the most poorly in this analysis. At a relative risk of 1.5 , their powers are considerably lower than the other methods. As the signal size increases, their power is lower up to relative risk of about 6 . However, in the interim, the empirical Bayes method seems to perform the best out of the three. At a relative risk of 2.5 it seems to improve considerably, while the SPRT catches up and surpasses the power of the empirical Bayes method at approximately 4 . Note that the power at 4 is high enough that these differences are not that telling.

These results are not entirely conclusive as there are some factors biasing the results. First of all when checking the observed Type one error rates, we get averages well above 0.05 for the Yates method which implies that the power is inflated. Thus, the Yates' method may not be as powerful as the comparison is showing. Other issues exist in that a number of the methods have extremely low observed Type one error rates, implying that their respective power is actually greater than observed. Thus, our comparison has some problems, and it is important to ascertain why the observed Type one error rates are not similar to the prespecified alpha value. 


\subsection{Significance levels}

The empirical Bayes quantile method, the empirical Bayes method with Roux's signal detection criteria, the SPRT, the Chi-Square Yates, and the PRR cutoff method have problems holding the prespecified significance level through simulation. It should be noted that throughout this discussion significance level and Type one error rate can be used interchangeably. These rates are determined by the procedure mentioned in section 6.3; we determine the proportion of times in each non signal cell that signals were flagged. Then we take an average across all these cells to determine the overall Type one error rate for each matrix of results. Ideally, these should be fairly close to 1,5 or $10 \%$ depending on the run, however for the methods listed above, this is not the case. Generally, in a hypothesis testing problem, the test holds the Type one error at the prespecified value through its critical value. Throughout this discussion we have seen critical values chosen based on common sense as opposed to mathematically determining the values that would properly fix the Type one error. Thus, this will be our starting point in finding a solution to this issue.

The empirical Bayes quantile method attempts to compare a statistic derived from a posterior distribution against a critical value. There has been discussion in the literature on this critical value, and the general consensus has been to compare the value $\lambda_{0.05}$ to one or two (van Puijenbroek et al., 2002; Szarfman et al., 2002; Roux et al., 2005; Hauben et al., 2005). However this value should be calculated from the following equation: $P_{H_{0}}\left(\lambda_{0.05}>k \mid n\right)=\alpha$. The critical value $k$ will ensure that the value of alpha is 
kept stable through the test. The same can be said about the other methods. The SPRT as described by Roux et al, uses a cutoff of 2.94 as a critical value. This number is determined by presetting both the Type one and Type two error at 0.05 . However, Wald states in his paper (Wald, 1945) that these inputs simply act as upper bounds for the errors and thus to specify a Type one error that holds the following probability must be evaluated: $P_{H_{0}}(\ln (2) \cdot a-E \geq k)=\alpha$ where $k=\ln \left(\frac{1-\beta}{\alpha}\right)$. Thus, we can pick either alpha or beta and fix that value, but the other error will be dependent on the overall value of $k$. In other words prespecifying $\alpha$ and $\beta$ will only assure that these errors are not surpassed by the test, to fix one of them requires solving for $k$ first. The same line of thinking applies for all the methods that did not achieve the Type one error rates in the simulation. One other fact that adds to this argument is the all the estimators transformed to approximate the normal distribution seem to have no problem preserving this rate. The critical values are generally known for the normal distribution, for example the confidence intervals for the IC are created using a standard normal statistic and as such the decision criteria will preserve the Type one error rates.

To deal with this issue we will look at the underlying set up of the data sets. Here each cell is distributed as a Poisson random variable with an unknown mean. Thus the critical value should derive from the Poisson distribution. For example, in the empirical Bayes method, the search for the signals depends on $\lambda$, however $\lambda$ is simply a piece of the unknown mean of the underlying Poisson, $n_{i j} \sim \operatorname{Poisson}\left(\mu_{i j}\right) \equiv \operatorname{Poisson}\left(\lambda_{i j} E_{i j}\right)$. 
Thus, if we examine this hypothesis testing problem from the perspective of the raw cell count we get $H_{0}: \lambda \leq 1=\mu \leq E$ and $H_{1}: \lambda>1=\mu>E$. However, the only method of testing $\mu$, is through the value of $n_{i j}$, thus we can instead evaluate, $P_{H_{0}}\left(n_{i j}>\mu_{i j} \mid E\left(n_{i j}\right)=E_{i j}\right)$. Note that the second probability essentially has $\lambda=1$ as its condition of being from the region in which the null hypothesis is true. Thus this probability can be expressed as $1-P_{H_{0}}\left(n_{i j} \leq \mu_{i j}=K\right)=1-\sum_{n_{i}=0}^{K} \frac{e^{-E_{i j}} E_{i j}^{n_{i j}}}{n_{i j} !}=\alpha$ where $K=\lambda_{i j} E_{i j}$. From our contingency table we already have the values of $E_{i j}$, and by solving the top of the sum to attain a value for $K$ satisfying $\alpha$ we can determine the critical value of $\lambda$ to maintain $\alpha$. Note that $K$ will be an integer and potentially cause the solution to this probability to never attain a value of exactly $\alpha$. However, we only need an approximate value to keep our test size in line with the other estimators, and as such this approximation is satisfactory keeping in mind that one should take the upper value of $K$ to keep the value of the probability less than $\alpha$.

This technique appears to be fairly sound; however in simulation we encounter similar issues. Also note that this general strategy can be applied to virtually all of these problematic methods; however in simulation they are still unable to achieve the nominal significance level. However, there is one major flaw with this method that is true across all of the faulty methods. The value of $E_{i j}$ is obtained from the data and although this method and others treat it as a fixed value it is in fact a random variable subject to 
sampling variability. To add further evidence to this hypothesis we will examine the Chisquare with Yate's correction method. This method is an accepted method in statistics and many simulations have validated its abilities to hold the Type one error rates. So it seems odd that in this application there are issues with this estimator. However the underlying theory behind this estimator comes from the Pearson Chi-square and is based on the squaring of a standard normal random variable, $\left(\frac{X-E(X)}{S D(X)}\right)^{2}=\frac{(X-E(X))^{2}}{\operatorname{Var}(X)}$. In our application, $\mathrm{X}$ is distributed Poisson and as such has the same expectation and variance. Thus, if we treat the values of $E_{i j}$ as the parameters of the Poisson distributed variables, and then if the estimates are off, the underlying distribution of the statistic will not necessarily be a Chi-square. To test this, simulations were done in which the percentage of signals were altered from run to run. Since the location of the signals in the data is randomized and the number of signals present in a row or column will significantly impact the value of $E_{i j}$, we would expect to see a noticeable effect on the value of the Type one errors associated with the Yate's statistic. As hypothesized these error rates increased in a similar fashion to the percentage of signals in the table. This implies that there is a direct correlation between the values of $E_{i j}$ and the Type one error rates further implying that the sampling variability in the expected frequencies is causing these problematic test statistics to not hold their size appropriately. Keep in mind that the methods that are holding that were previously stated to use normal transformations are 
not using the $E_{i j}$ 's to perform these transformations and as such their impact on the statistic and subsequently on the hypothesis testing problem is minimized.

Therefore, it seems reasonable to exclude these measures from comparison. A direct power analysis similar to the one in section 6.4 only allows us to compare the methods that have similar sizes. Otherwise our conclusions will be inappropriate and our ability to rank the methods will be inadequate.

Finally, though some of the methods discussed in section 6.5 may not achieve an ideal Type one error, some of these tests are still valid in that they achieve alpha values less than the prespecified one. The methods are the EBs, the SPRT and the PRR cut off and it seems important to mention that they are still valid as signal detection methods. The conclusions of section 6.4 show that these methods in fact do a decent job with respect to power. Although we cannot assess them in comparison to other methods due to the alpha level issue, these tests appear to have high specificity and as such we can conclude that their power is not inflated due to inappropriate critical values. Thus, while we will not discuss the methods further in the analysis, it is important to recognize that they are potentially valid methods of signal detection and more research is required to solidify the Type one error rates and to decide on their relative abilities in this field. 


\subsection{Power Analysis}

Keeping the results in section 6.5 in mind we will conduct another power analysis this time only of the methods that have Type one error rates which hold under simulation. These methods are the adjusted residual technique, the PRR confidence interval, the ROR confidence interval, Yule's Q's confidence interval and both WHO methods. Thus we are comparing six methods across three methods of signal size (relative risk) generation and at three levels of confidence. To begin, we will look at the power curves generated across fixed values of relative risk at $5 \%$ confidence. The actual points on the graph are removed to make them easier to read and we will simply focus on the Lowess curves.

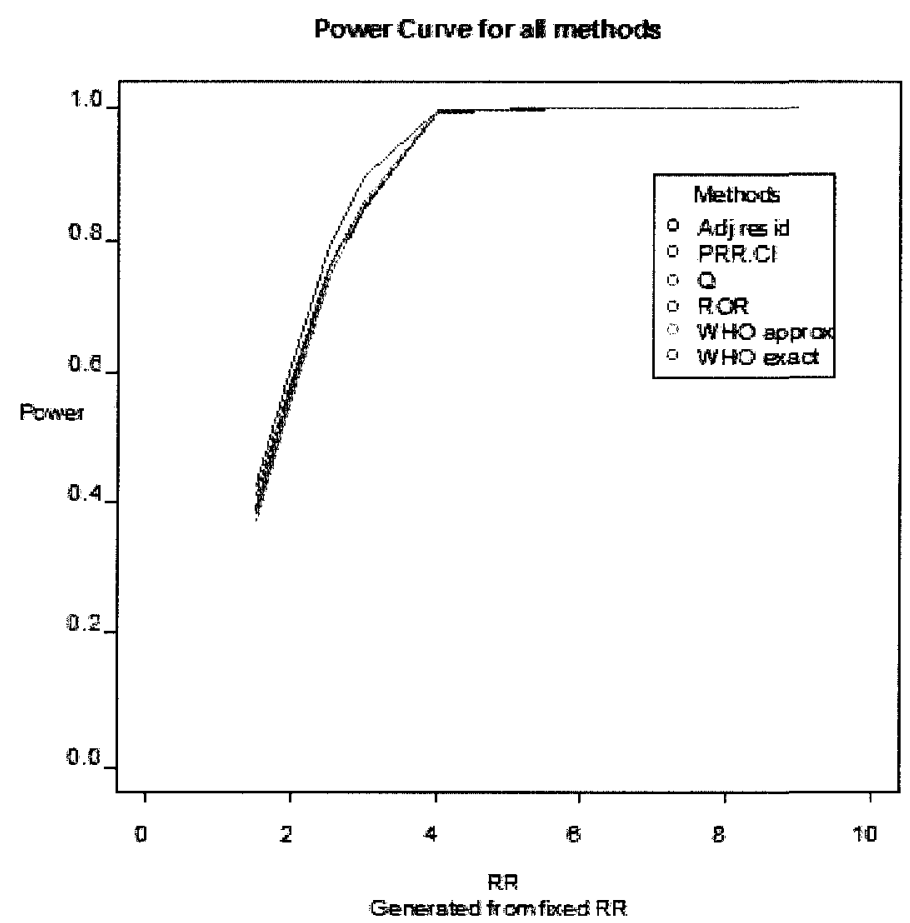

Figure 6.6.1 - Power versus RR for the remaining methods at $5 \%$ significance 
It appears that the methods all seem to classify quite well especially at approximately a relative risk of four or higher. A relative risk below two is difficult to detect; as the power dips below $80 \%$. Between two and four, the WHO exact method seems to do a slightly better than the others, however the difference is so small that it is hard to conclusively say that it is the best estimator. In fact, it could simply be due to the approximation that occurs in Lowess.

So it appears that our estimators have proven to classify quite nicely in mid to high ranges of relative risk, perhaps their performance will change with the introduction of variability to the signal sizes. We have uniformly and exponentially-generated relative risks and we can perform the same analysis on both sets of data to observe the impact.
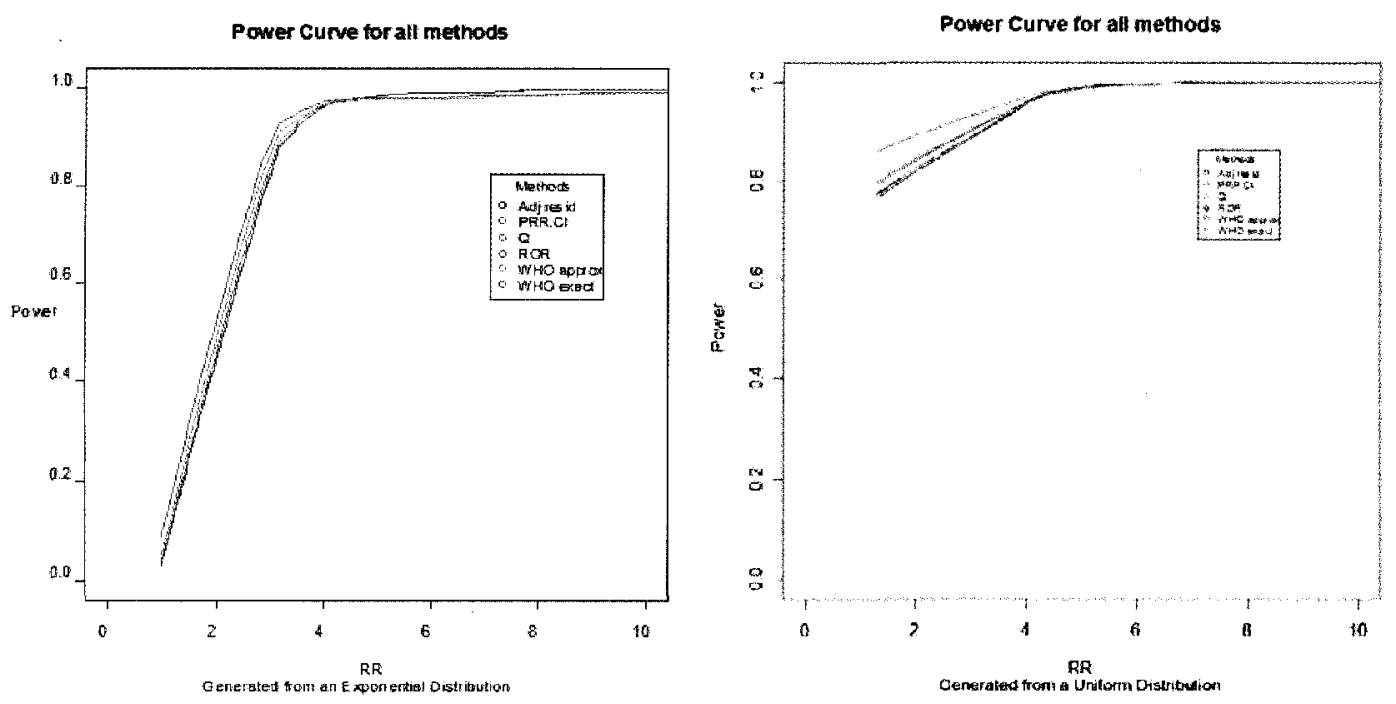

Figure 6.6.2 - Power comparison of remaining methods at $5 \%$ significance 
The graphs illustrate that power seems to be affected by difference in the underlying variability affecting the size of signal. The uniformly generated signal size data seems to have extremely high power at lower levels of relative risk compared with the fixed and exponential data. Note again that the Lowess approximation is causing the power to appear higher than it actually is and as such we will examine a plot of the original points for the uniform data and for completeness the exponential as well.
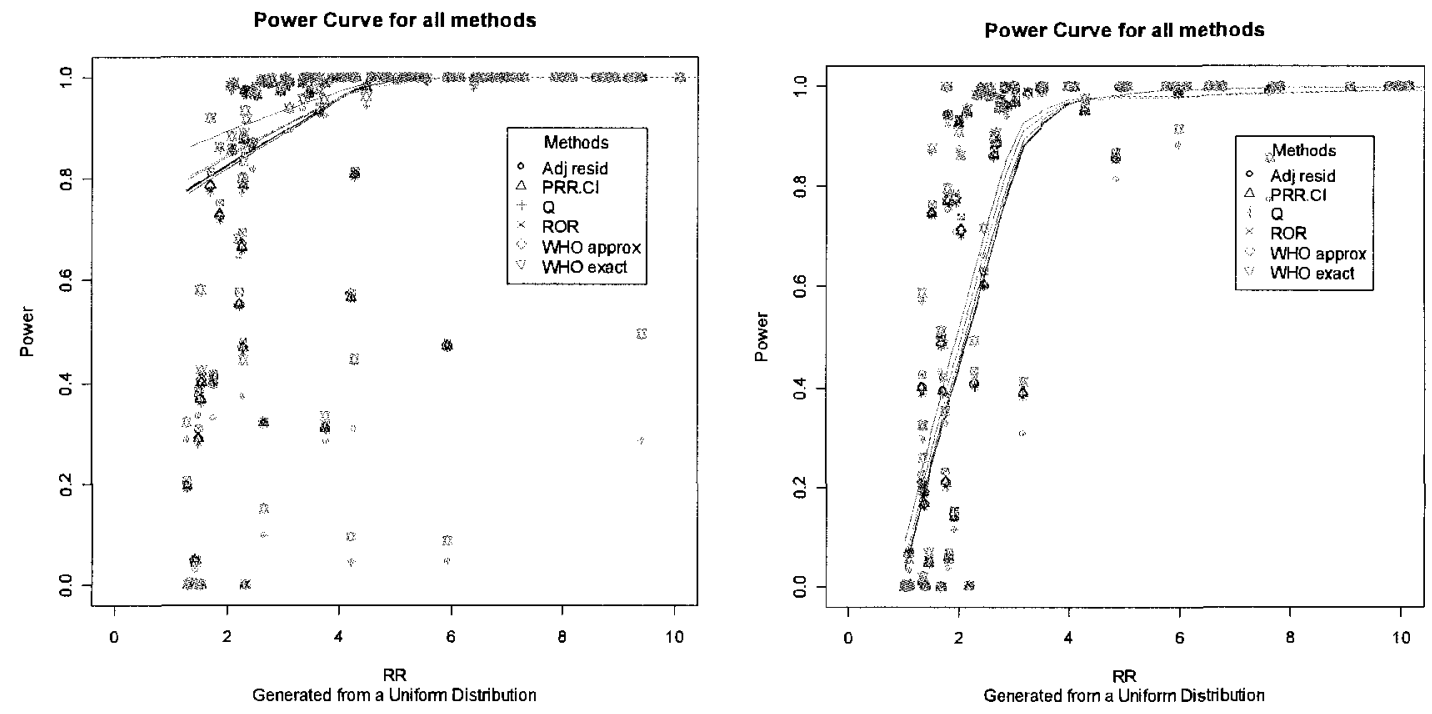

Figure 6.6.3-Actual data for power comparison

As expected there are a large number of points at low power for relative risks from 2 to 6 on the uniform plot. The Lowess method is not picking up on these as being very strong values and as such the approximation is inflating the power. That being said the comparison of methods under this distribution is still somewhat valid since the distribution of points in this area seems to come from all the estimators. Once again the 
WHO exact appears slightly higher than the rest and that holds true for both plots. The rest of the methods appear to perform at a relatively similar rate of classification.

The final comparison involving changes to the underlying distribution generating the size of the signal will be examined by exploring individual methods and the data from each of the three underlying distributions (fixed, exponential and uniform). For this analysis we will start by examining the ROR:

Power Curve for ROR at 5\%

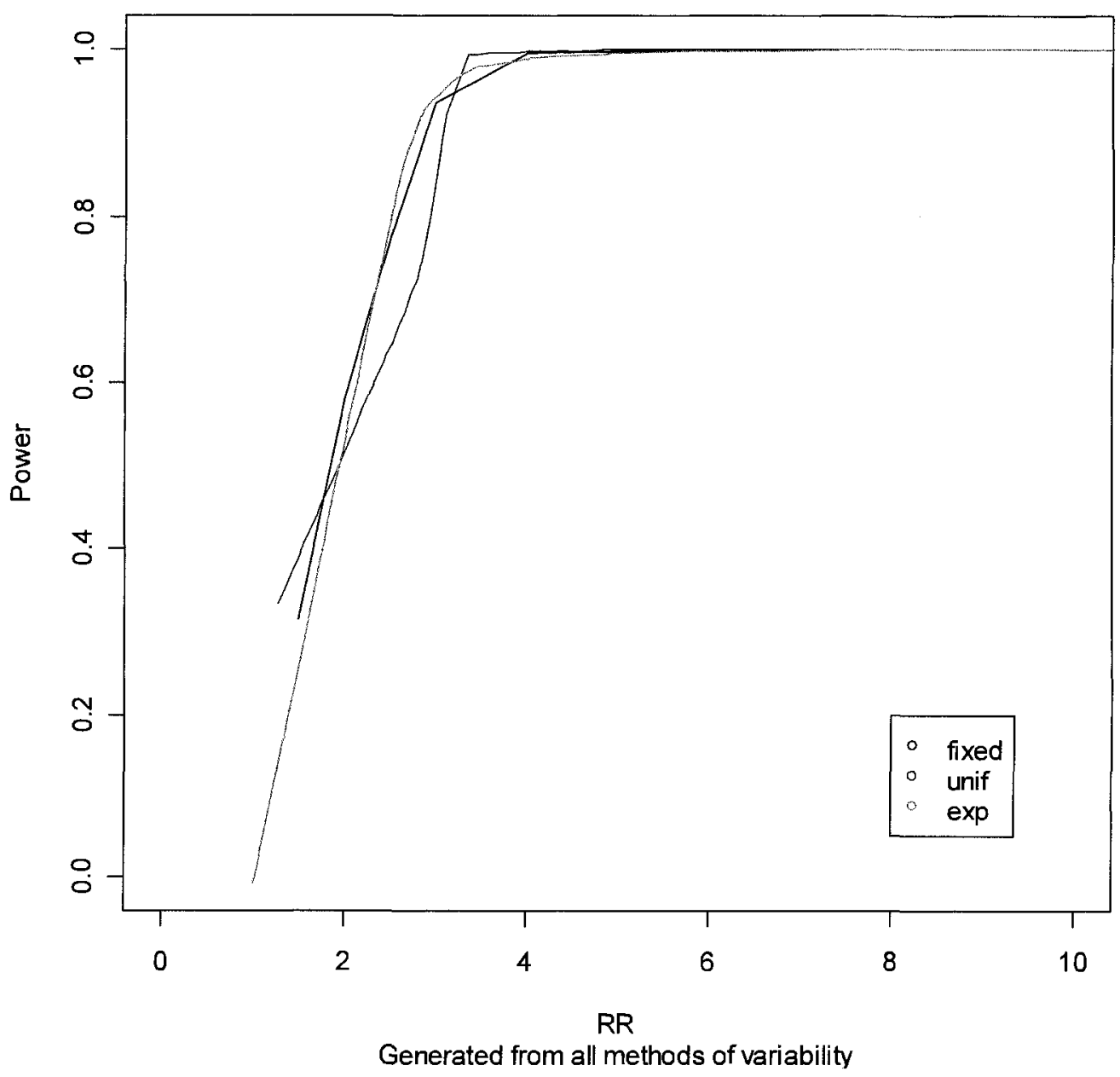

Figure 6.6.4 - Power comparison for ROR at $5 \%$ significance across three types of variability 
It seems that the fixed and exponential curves are fairly close together except as the relative risk approaches 3 where the exponential seems to beat out the fixed by a small amount. In this plot the amount seems too small to conclude that this is in fact the case, however this observation is fairly consistent across all of the estimators. As far as the uniform goes, a number of the estimators show a power curve that is lower than the others up until around 4 and then it surpasses the other signal size generation methods briefly as the relative risk increases. This observation applies to all the estimators except for the WHO approx method and some of these curves are displayed below.
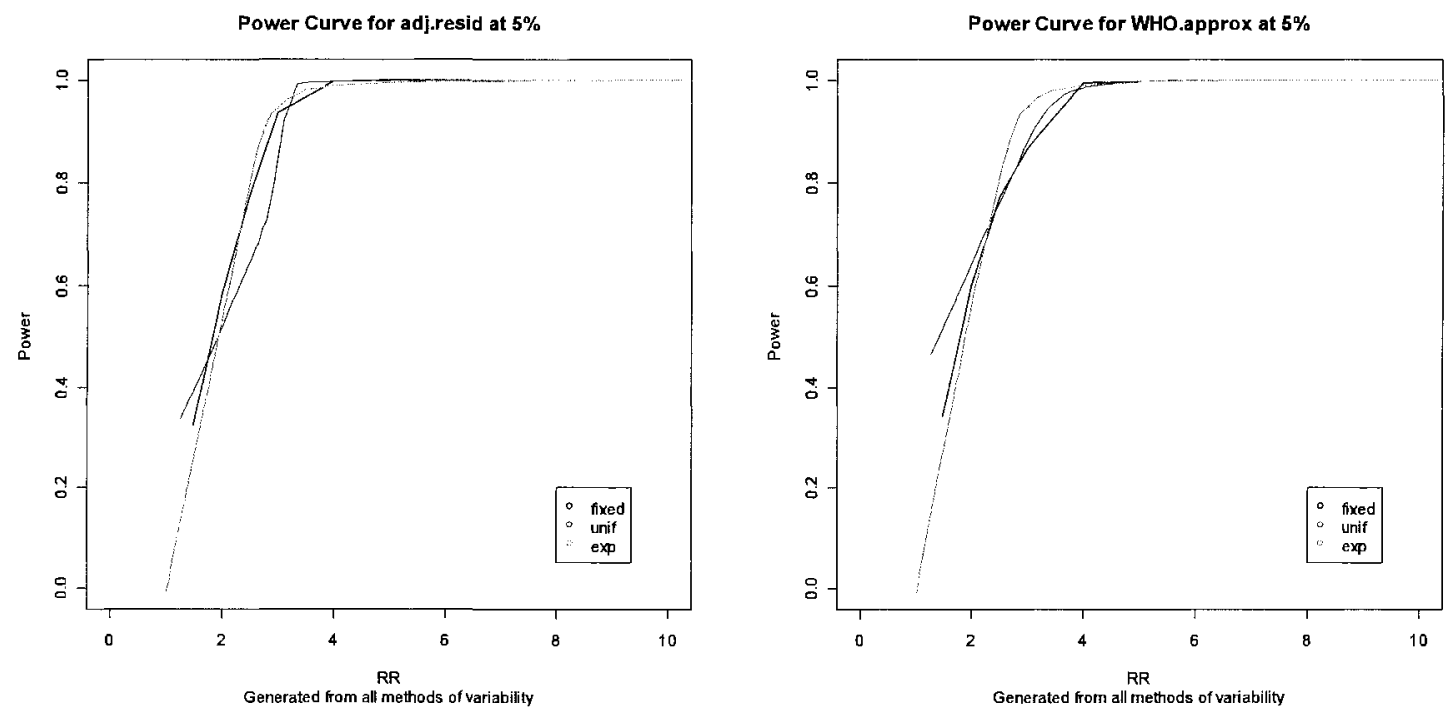

Figure 6.6.5 - Power comparison with respect to underlying variability for Adjusted Residual and WHO approximation methods

Thus, we can conclude that changing the underlying variability responsible for the size of signal has an effect on power however more research must be done. 
Now that we have examined the performance of the estimators with respect to the signal size and the methods of generation, we can begin to examine how altering the significance levels can effect power. To do this we will fix upon a single signal detection method and a single method of signal size determination. The reason for this simplicity is to avoid overanalyzing data that says the exact same conclusion. We are curious about a single question in this section and that is, how does altering the significance level affect the performance of the estimator? Thus, we will pick an estimator that seemed to perform fairly well and possesses an observed Type one error rate that is reasonably close to the prespecified values. For this analysis we will choose the reporting odds ratio as it meets the previous criteria. Also we will examine the curve where the size of signal was generated from a uniform distribution. 


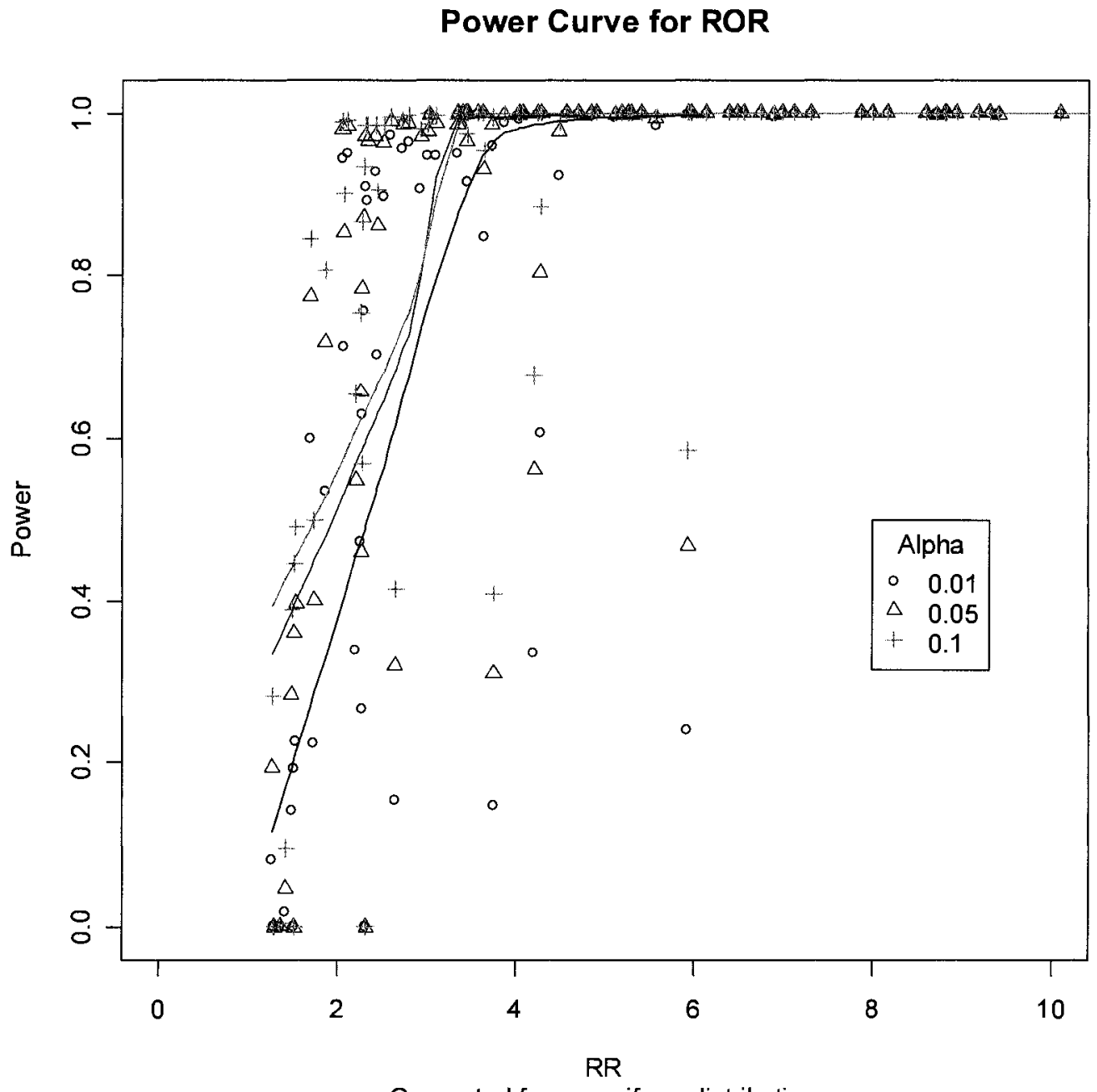

Generated from a uniform distribution

Figure 6.6.6 - Power comparison for ROR across three levels of significance

As is seen in figure 6.6.6 it seems clear that altering the alpha level will in fact alter the power as is expected; as the alpha level increases, so does the power. Below is a close up of the actual data between this region. 
ROR generated by a uniform for RR between 2 and 3

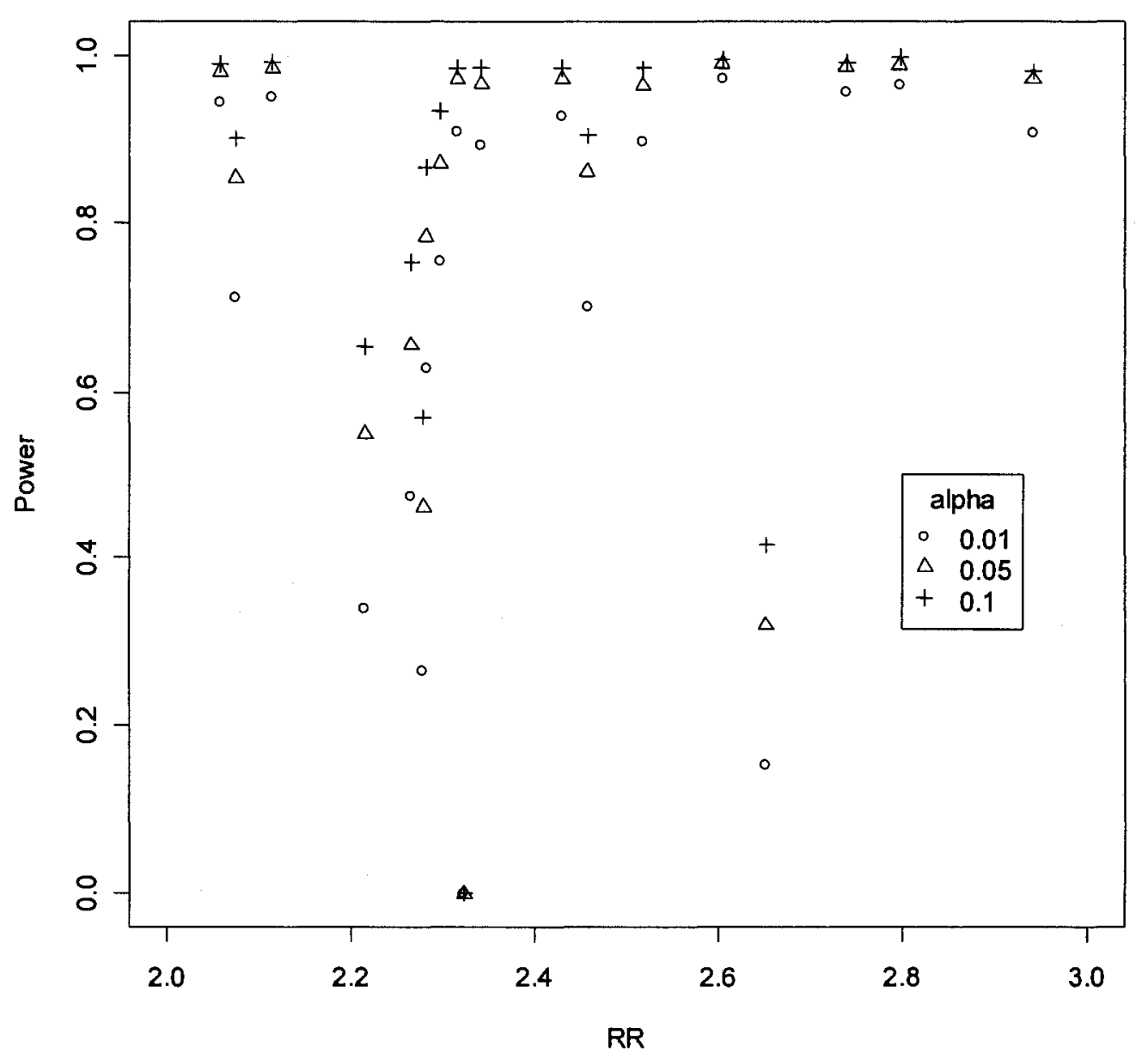

Figure 6.6.7 - Power comparison for ROR of three significance levels within a range of $R R=[2,3]$

Again it can be seen that the higher alpha level ' + ' has higher power over the alpha 0.05 points. Another way to tell is to examine the average for each set of points in this region. We get 0.7961053 for the alpha 0.05 mean between a relative risk of 2 and 3 , and 0.8355263 for the alpha 0.1 power values. Finally, keep in mind that this comparison of significance levels holds across all of the methods and methods of generating signal size. 


\subsection{Empirical Bayes Roux}

Finally, we are left with the empirical Bayes method using Roux's criteria (Roux et al., 2005) for signal detection. Thinking back to the theoretical discussion on this method, Roux's criteria for signal detection (3.2.9) looked at the probability that $\lambda_{i j}$ comes from a distribution such that the expectation exceeds one. They do this by weighting each of the posterior weights termed $Q_{n}(3.2 .4)$ by a value represented by $\delta_{k}$, where $\delta_{k}$ takes on a value of one if the posterior expectation of the $k^{\text {th }}$ piece of the mixture has an expectation greater than one and zero otherwise. The authors assert that this will in fact produce a situation in which this probability is comparable to 0.5 to determine whether a signal exists. This method essentially constructs a hypothesis test in which we are weighting the probabilities of belonging to certain regions and comparing them against each other.

The reason that this method needs to be discussed separately from the others is due to the reason behind the lack of ability to fix the Type one error rate. By the authors criteria, we are in a situation where the null hypothesis should be rejected if $Q_{n} \delta_{1}+\left(1-Q_{n}\right) \delta_{2}>c$ where $c=0.5$. This requires a form for the null distribution of the left hand side of the inequality to determine the value for $c$. Note that these $\delta_{k}$ 's are

effectively Bernoulli random variables with $p_{k}=P\left(\frac{\alpha_{k}+n_{i j}}{\beta_{k}+E_{i j}}>1\right)$ and we will assume that the $Q_{n}$ 's can be treated as constants for simplicity's sake. If we are unable to solve for $c$ with this constraint then allowing for randomness in the $Q_{n}$ 's will make the 
possibility of this critical value being analytically solved even more unlikely. Here, $p_{k}$ is a probability that depends on the observed and expected cell counts in the dataset. Since the posterior distribution shifts from cell to cell with respect to the parameters, this probability is going to depend on the data and as such attempting to derive a distributional form for a weighted sum of these Bernoulli's is extremely difficult. This is especially true under the condition of the null region since this region's definition depends on these probabilities. The null region can be expressed as $E B p>0.5 \Leftrightarrow\left\{\delta_{1}=\delta_{2}=1\right\} \cup\left\{\delta_{1}=0 \cap Q_{n}>0.5\right\} \cup\left\{\delta_{2}=0 \cap\left(1-Q_{n}\right)>0.5\right\}$ and from the first event in this space we can see that it is dependent on the probabilities of success in the Bernoulli random variables. Thus, it is impossible to fix this Type one error rate without adequate knowledge of the $p_{k}$ 's.

Examining the Type one error rates for this method from the simulation we get an average value of $29.212 \%$ for the uniform distribution set of results, $36.75 \%$ for the exponential and $24.9 \%$ for the fixed relative risk method. Below are the power curves for the three forms of variability as follows. 

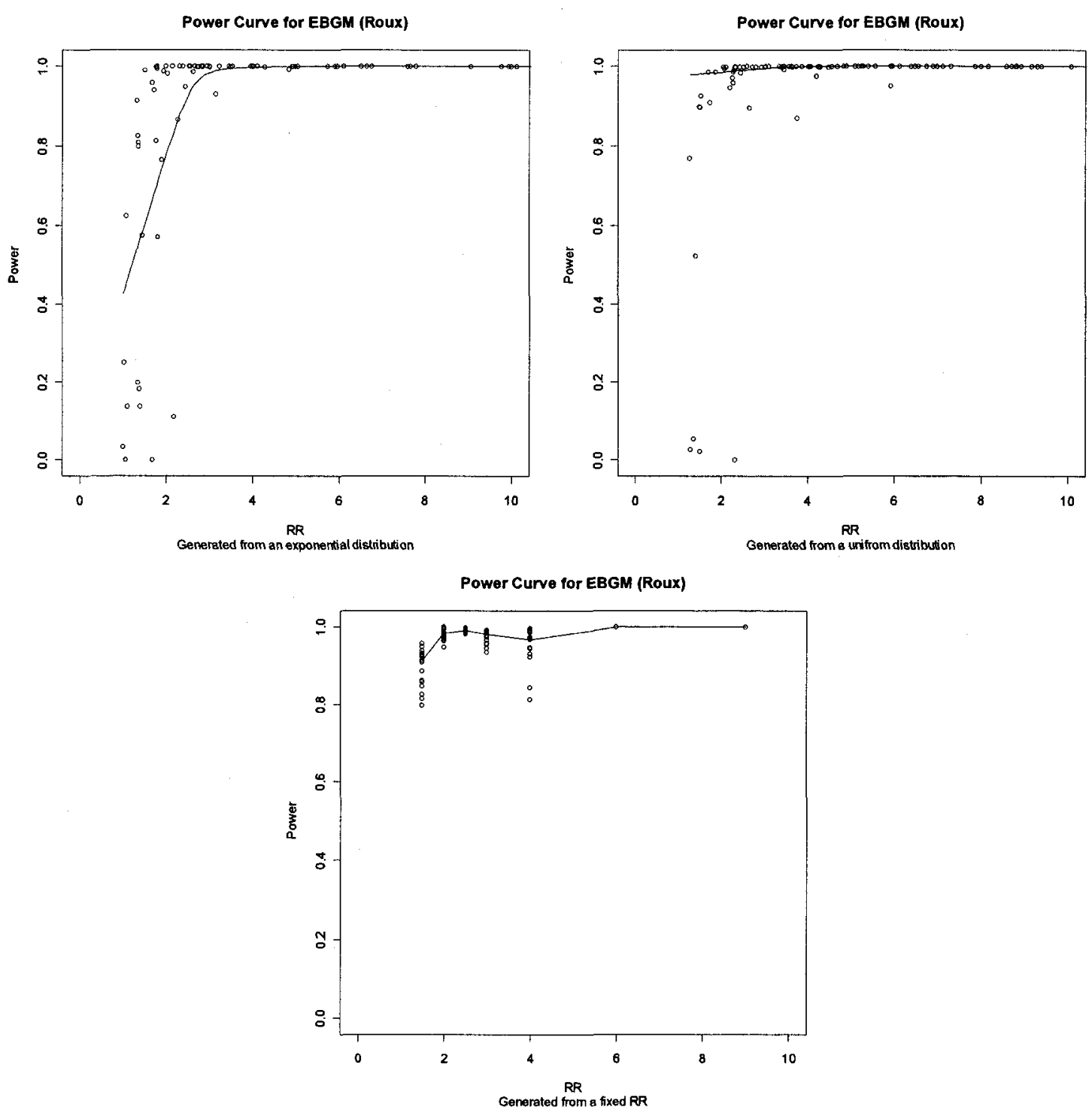

Figure 6.7.1 - Power versus RR for EBGM (Roux) across three types of underlying variability

Note that the exponential and uniform methods yield extremely inflated results with a large amount of points of almost $100 \%$ power at approximately a relative risk of 2 . The fixed method is even more inflated where there does not appear to be any points below 
$75 \%$. Thus, this method is over flagging signals at an extremely high rate and although it will more than likely flag the so-called true signals quite effectively, they will be buried among a large number of non-signals. Thus, this signal detection criteria is unadvisable unless the algorithm is altered to fix the Type one error rate at an appropriate value. 


\section{Chapter 7}

Conclusion

When attempting to make conclusions about the variety of signal detection methods discussed in this paper, we run into difficulties. To begin, all of these estimators are plagued by a common shortcoming that is out of their control: the data collection methods. In the theoretical sections in Chapters 2, 3 and 4, some of these issues were brought up for discussion. Specifically, the PRR, ROR and Yule's Q suffer from the assumption that the data set being examined is an adequate representation of real life. Since these methods rely on the makeup of the data matrix to draw inference about the background frequency levels of these drug/ADR pairs, they are heavily influenced by the data collection method. The SPRT requires prior knowledge of the distribution of a signal to appropriately set up hypotheses and subsequently a likelihood function. Finally, the Bayesian methods make assumptions on the makeup of the 'true' population by imposing the specifications of prior distributions. These methods may be the least impacted by poor data collection methods in that they apply probability distributions and allow them to be updated. The adjusted Pearson residual method, proposed in this discussion for use in pharmacovigilance, uses a standardizing transformation to effectively shift any distributional issues with the underlying data to attempt to deal with this problem. This technique of using transformations to assist in lowering the impact of 
the biasing effects of the data is also used by some of the previous methods and the results are favourable.

Ignoring the data collection issues, we can then proceed to discuss the performance of the estimators in further detail. The purpose is to see whether they flag situations in which the correlation between a drug and ADR is larger than would be expected due to chance alone. The estimators seem to accomplish this at high levels of relative risk, or when the signal is large enough. The issue of how well these estimators perform is more of a concern in the lower levels of signal size. We have seen that the Bayesian methods were constructed for this issue in that their use of priors to detect small signals can be quite effective. The WHO methods performed among the best in the power analysis conducted here, and the empirical Bayes method did relatively well in the preliminary power analysis in section 6.4. Even though its Type one error rate was estimated quite lower than what was specified, this allows us to infer that the power observed for this method was not biased upwards and as such we can still infer that it is a good method, although we cannot compare it with other estimators. Again a point that was brought up in the theoretical discussion is that these priors are potentially incorrect and the Bayesian method really shows its strengths in longer term surveillance, as the updating of the methods causes the posterior to get closer and closer to reality as more data is collected. Coupled with the observation in the previous paragraph that the dependency of the methods on the raw data is minimized allows for fairly good forms of signal detection all around. The PRR, ROR and Yule's Q do fairly well at finding signals 
at lower levels of relative risk as well. The adjusted residual technique seems to perform at similar levels to these previous methods. Again, the remaining methods are difficult to compare due to the Type one error issue discussed in section 6.5 .

In simulation we observed that the estimator's abilities to classify are affected by the underlying variability in signal size generation. Practically this means that the hypothetical probability distribution attached to the size of dependency between drug and ADR will impact on the ability for these estimators to flag the appropriate drug/ADR pairs. This is an area of research that needs more work. Another question that can be pondered is the altering of the significance levels. As was seen in the simulation adjusting these levels altered the power and detection abilities of the methods. The question is one of social impact in this case. By increasing the magnitude of Type one error, we improve power which practically means that we will be telling researchers to study more drug/ADR pairs than may be needed under a lower significance level, however we will be accurately flagging more true signals. This becomes a balancing act between resources and signal detection.

In this discussion we pointed out a number of areas in which conclusions appear to be questionable. The formula for the logarithmic variance of the PRR, the variance of $\log$ lambda in the empirical Bayes approach, the fixing of the alpha levels in simulation and Roux's signal detection criteria are all areas that require some further study. While a correction to the formula for the logarithmic variance of the PRR was proposed in this paper, the covariance term in Gould's variance equation requires the development of the 
joint distribution of two log gamma random variables. Fixing the alpha levels require an evaluation of an appropriate null distribution for the SPRT and empirical Bayes methods. Also coming up with a random effects type model may alleviate some of the issues found with random expected frequencies which would help the methods proposed in section 6.5 accurately determine appropriate critical values. After these issues are corrected, a power analysis will be a much more useful tool for comparison of the methods.

In the broader sense the future of pharmacovigilance has already begun in the development of multi item comparison methods expanding on the methods proposed in the literature. Examining multiple simultaneous correlations is one way this field has expanded. Fortunately, the field has also expanded in data collection and we are beginning to see complete patient data from hospitals and health organizations. This data will remove a number of the issues plaguing the methods studied in this paper. Finally, an interesting piece of research and development surrounding passive pharmacovigilance could be the development of simultaneous signal detection techniques. For example, allowing a number of these methods to classify at the same time and applying the results to a multivariate probability distribution could yield a much stronger method of signal detection.

In conclusion, this field of pharmacovigilance is full of problems and glitches that require fine tuning and development over time. However, this field has had success in pre-emptively flagging signals which lead to further research from the pharmacologists and has resulted in subsequent action. For every negative characteristic there appears to 
be a positive outcome in this field of research. As long as pharmacovigilance experts continue to develop and rethink the problem of the search for signals, this ever-evolving field will become stronger and stronger. The future of pharmacovigilance will manifest itself in the improved abilities of experts to flag these potentially dangerous interactions, whether it is in passive surveillance or in the new more comprehensive patient data. 


\section{Appendix 1}

\section{Vioxx Example}

\section{Code to generate Vioxx data}

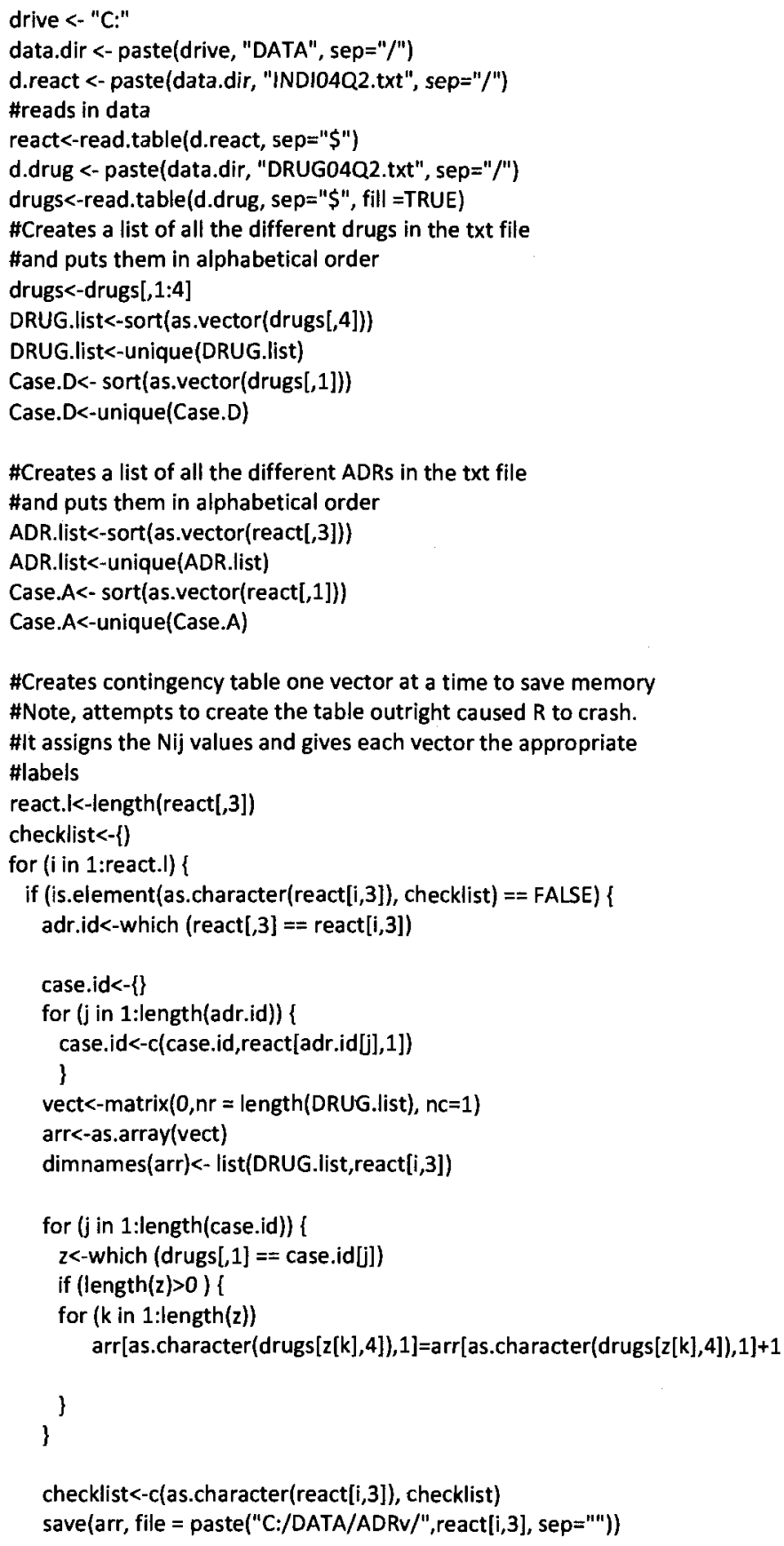




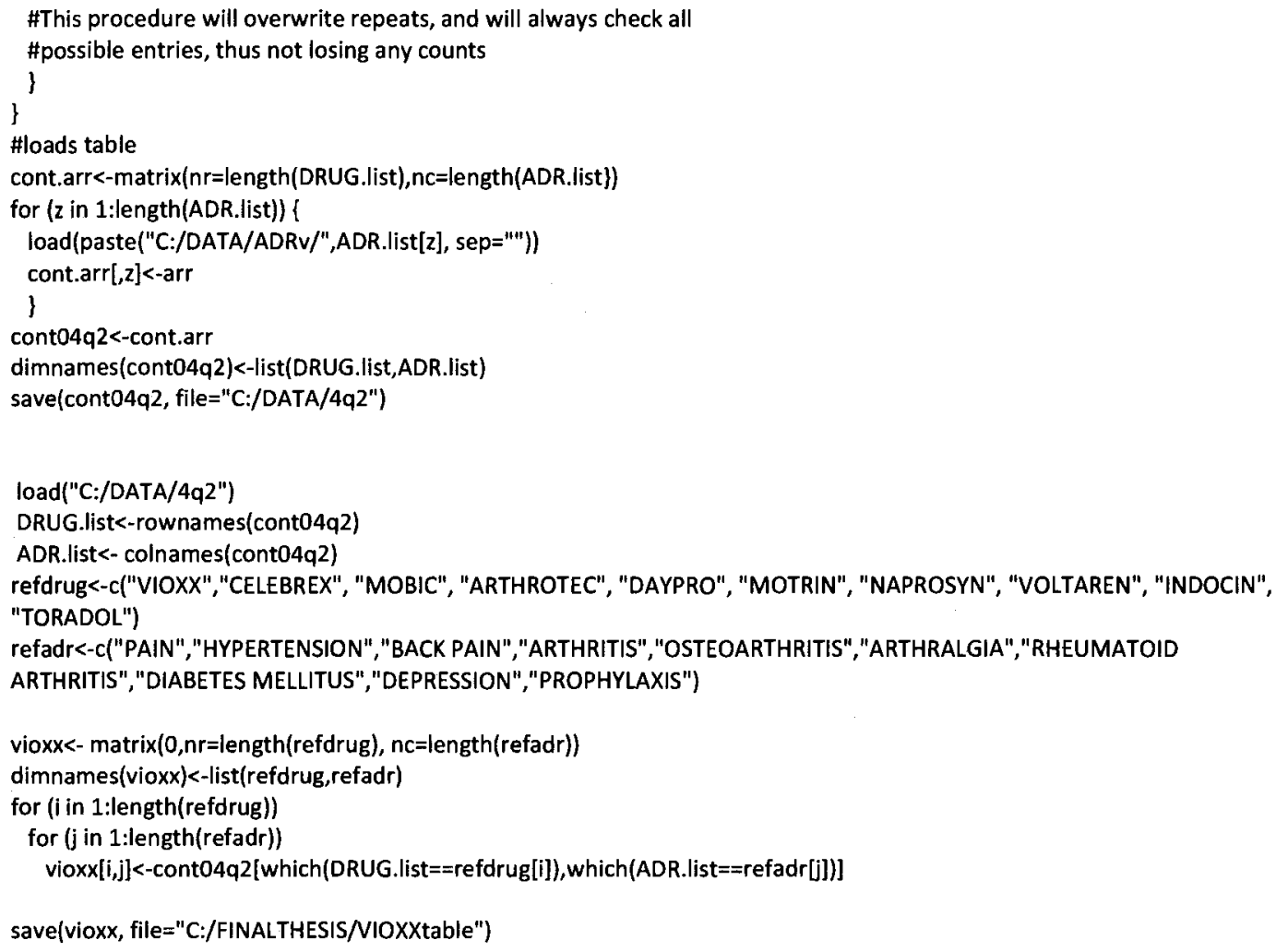




\section{Vioxx signals flagged}

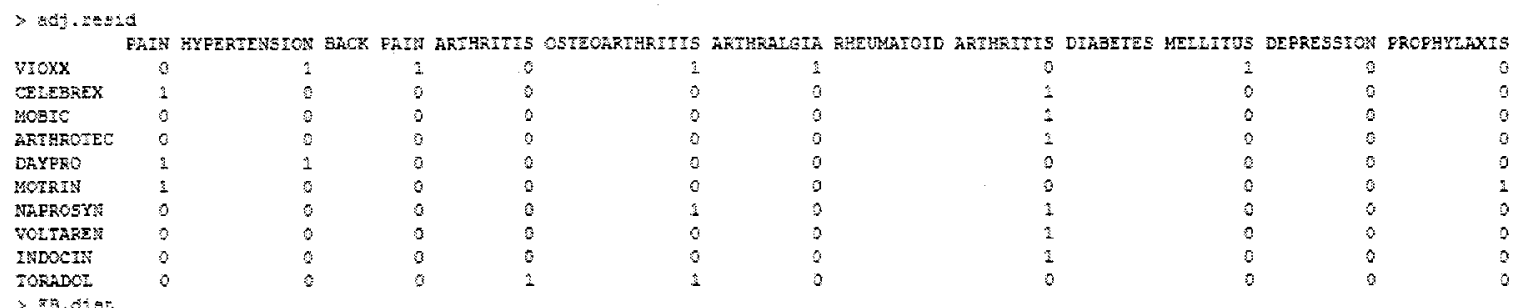

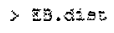

rovy

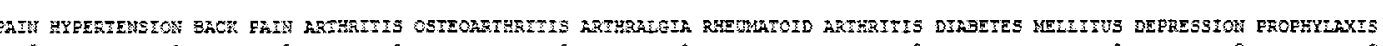
CELEgax weresc ARTMAOTEC DAYPRO Morez: Yorkz: FARCSY: VOLTARE: introcts

TORALOE

$>$ I3.

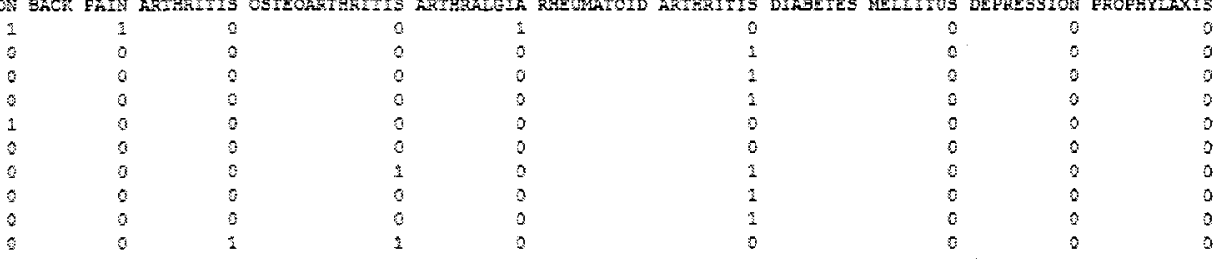

vasx

Eñ

(1)

1
1
0
0
0
0
0
0
1

TIS FRTARALE
1
0
0
0
0
0
1
0
1
1

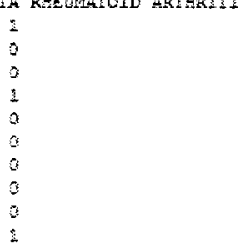

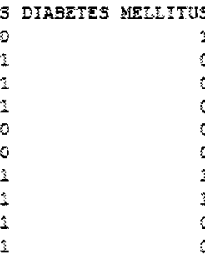

TORHDOL

Fet.

\begin{tabular}{|c|c|c|c|}
\hline 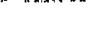 & gary & WYFERTENSIOA & BACE PAIM \\
\hline $\operatorname{moxx}$ & 8 & $\stackrel{1}{1}$ & 3 \\
\hline CEIEEREX & $\hat{i}$ & 0 & 0 \\
\hline YOBJE & 0 & $a$ & 0 \\
\hline AETREOTE & 0 & 0 & 2 \\
\hline DAYEPO & $\vdots$ & $\hat{1}$ & 6 \\
\hline MOTRIY & 1 & 0 & 0 \\
\hline WAFROSYY & 2 & $a$ & 0 \\
\hline YoLEASE: & 2 & 0 & 0 \\
\hline zhoosm & $a$ & 0 & 0 \\
\hline TOREXOLE & 2 & 0 & 0 \\
\hline
\end{tabular}

TORAROL

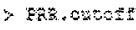

0
0
0
0
0
0
0
0
0
1

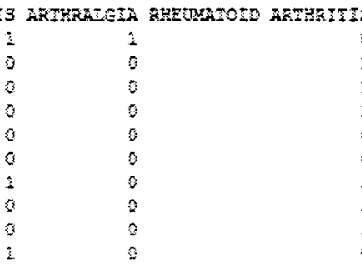

vioxx

CELEBhr

ARTBKORE

ARTEROTO

DAYFRO

NAEROETX

IOETAREM

FURATC:

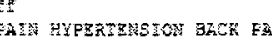

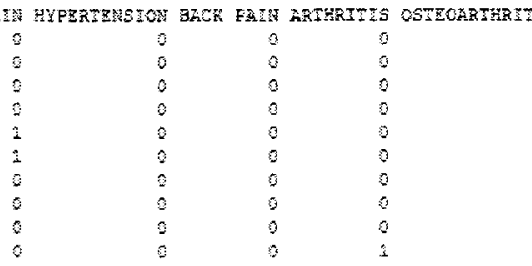

0
0
0
0
0
0
0
0
0
0

$>\mathrm{Q}$

VICXX
CELIEREX

Moste

ARTAROTEC

DAYFRO

MOTRIM

MARTOST:

morjare:

IMDOET:

TORADOE

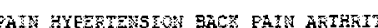

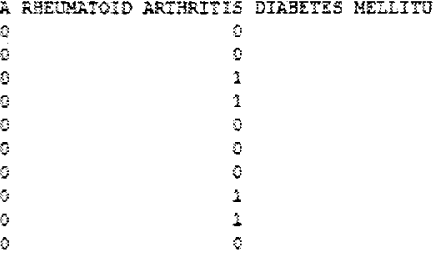

3
0
0
0
2
2
2
0
1

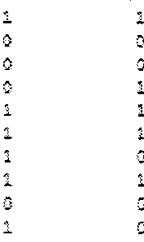

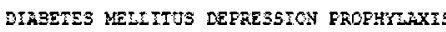
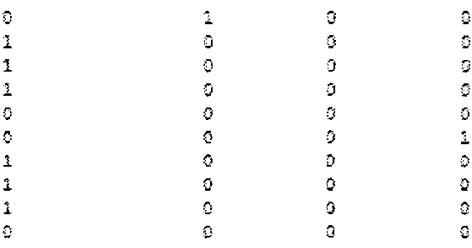

$$
\begin{aligned}
& 0 \\
& 0 \\
& 0 \\
& 0 \\
& 0 \\
& 0 \\
& 0 \\
& 0 \\
& 0
\end{aligned}
$$
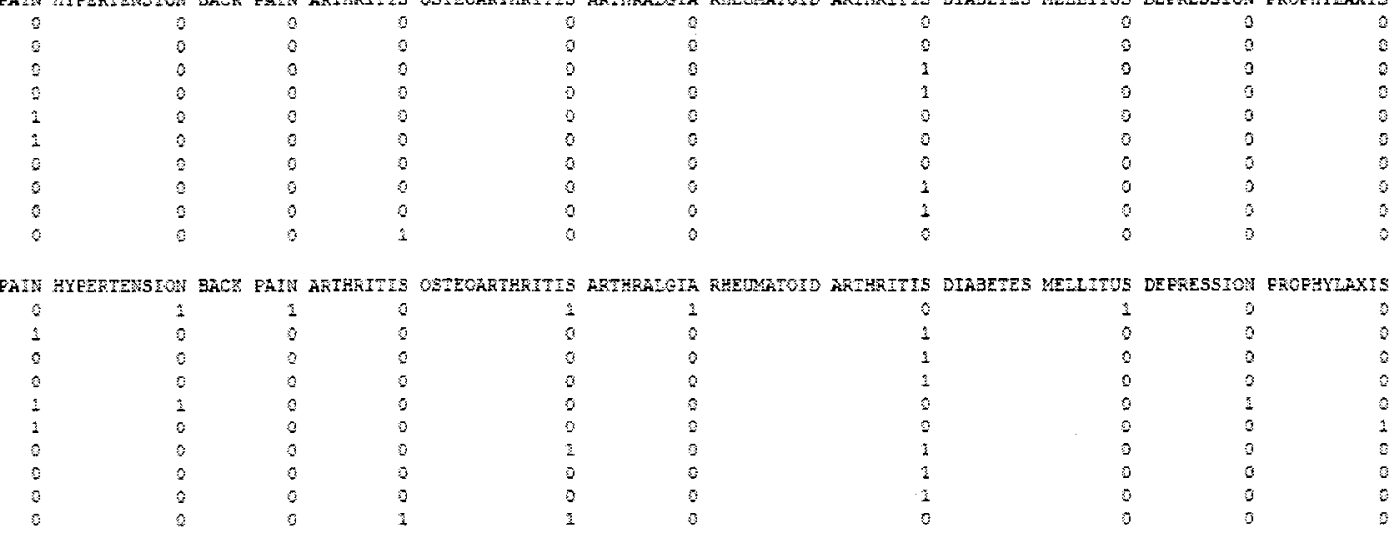

$\begin{array}{lll}0 & 1 & 0 \\ 0 & 0 & 0 \\ 0 & 0 & 0 \\ 0 & 0 & 0 \\ 1 & 0 & 0 \\ 0 & 0 & 0 \\ 0 & 0 & 0 \\ 0 & 0 & 0 \\ 0 & 0 & 0 \\ 0 & 0 & \end{array}$

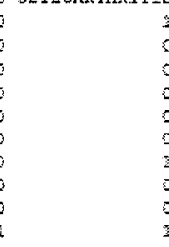
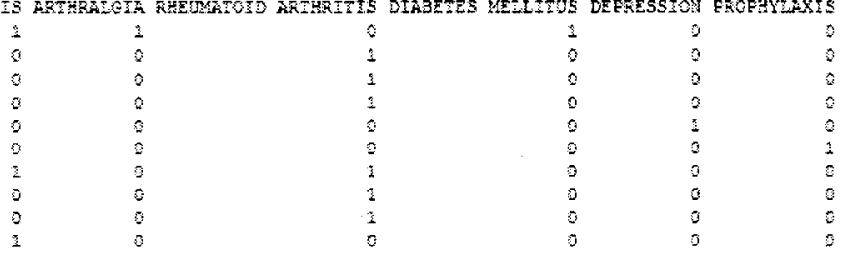


\begin{tabular}{|c|c|c|c|c|c|c|c|c|c|c|c|}
\hline tor & BDTS & HYF GTRETO & $B=\mathrm{Cr}$ & & & & & & & & \\
\hline y3oxx & 8 & $i$ & & $\hat{3}$ & 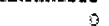 & 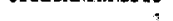 & 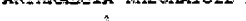 & 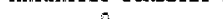 & 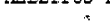 & - & \\
\hline CELEBREX & $\hat{i}$ & $n$ & & 3 & 3 & 3 & 3 & 3 & 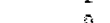 & 3 & 3 \\
\hline Mose: & 0 & 2 & & 0 & 0 & 0 & 0 & 1 & 0 & 0 & 3 \\
\hline RRTRORE & 0 & 0 & & 0 & 0 & 0 & 0 & 1 & 0 & 0 & s. \\
\hline DAYERO & $i$ & 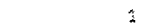 & & 0 & 6 & $a$ & 5 & 0 & $a$ & 5 & 5 \\
\hline MOThN & $i$ & 0 & & 0 & $a$ & $a$ & 0 & 0 & 0 & 0 & i \\
\hline NăFROSY⿱ & 2 & 0 & & 0 & 0 & 1 & 0 & 1 & 0 & 2 & 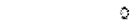 \\
\hline WLTAREM & 0 & 0 & & 0 & g & $\mathrm{a}$ & 0 & 1 & 0 & 0 & 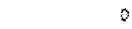 \\
\hline IMDOEII & 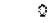 & 0 & & 0 & 0 & 0 & 2 & 1 & 0 & 0 & 0 \\
\hline TOEAEOL: & a & 0 & & 0 & $i$ & 3 & $\hat{s}$ & $\mathrm{~s}$ & 0 & 3 & t \\
\hline
\end{tabular}

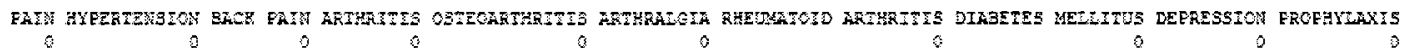

vexx

CELEgR

RRTEROTE

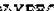

uor

MAFROSH

VORARES

IMPXTEN

TORADOL

-

$\begin{array}{ll}0 & 0 \\ 0 & 0 \\ 0 & 0 \\ 0 & 0 \\ 0 & 0 \\ 0 & 0 \\ 0 & 0 \\ 0 & 0 \\ 0 & 0 \\ 0 & i\end{array}$

$\begin{array}{ll}0 & 0 \\ 0 & \\ 0 & \\ 0 & 0 \\ 0 & 0 \\ 0 & 0 \\ 2 & 0 \\ 0 & 0 \\ 3 & 0 \\ 1 & 0\end{array}$

$\begin{array}{ll}0 & 0 \\ 0 & 1 \\ 0 & 1 \\ 0 & 1 \\ 0 & 1 \\ 0 & 1 \\ 0 & 1 \\ 0 & 1\end{array}$

0
1
1
1
0
0
1
1
1
0

$\begin{array}{ll}0 & 0 \\ 0 & 0 \\ 0 & 0 \\ 0 & 0 \\ 0 & 0 \\ 0 & 0 \\ 0 & 0 \\ 0 & 0 \\ 0 & 0\end{array}$

$\begin{array}{ll}0 & 0 \\ 0 & 2 \\ 0 & 0 \\ 0 & 0 \\ 0 & 0 \\ 0 & 0 \\ 0 & 0 \\ 0 & 0\end{array}$

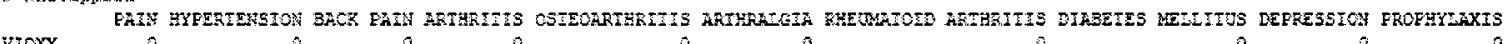

\begin{tabular}{|c|c|c|c|c|c|c|c|c|c|}
\hline $\operatorname{vroxx}$ & 8 & 0 & 0 & 0 & 5 & 0 & 0 & 0 & 0 \\
\hline $\operatorname{cec} 58 \mathrm{x}=\mathrm{x}$ & 0 & 0 & 0 & 0 & 0 & 0 & I & 0 & 0 \\
\hline mose & 8 & 0 & 0 & o & 0 & 0 & 1 & 3 & 3 \\
\hline AETHATEC & 0 & 0 & 0 & 0 & 0 & $\vec{i}$ & 1 & 0 & 0 \\
\hline EAYERO & 1 & $\frac{1}{2}$ & 0 & 0 & 0 & 0 & 0 & g & 2 \\
\hline WTRRIN & 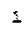 & 0 & 0 & a & $a$ & 0 & 0 & 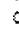 & B \\
\hline SG.FROSYM & 3 & 0 & 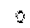 & 0 & 1 & 0 & 1 & 0 & 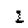 \\
\hline VOLEHRE: & 0 & 0 & 0 & 0 & 0 & $a$ & 3 & 3 & 0 \\
\hline IMLOCEN & 0 & 0 & 0 & $a$ & $a$ & 2 & 3 & 8 & 0 \\
\hline TORADOE & 0 & 0 & 0 & $i$ & 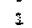 & 0 & 8 & a & 0 \\
\hline
\end{tabular}

Tho exact

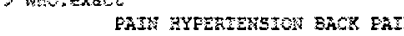

Troxx

CEI ESER

MOEIC

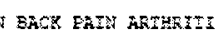

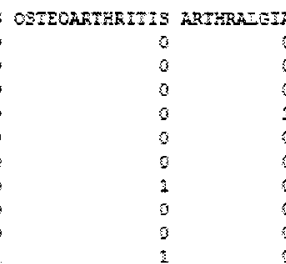

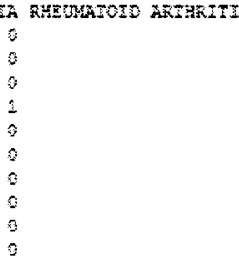

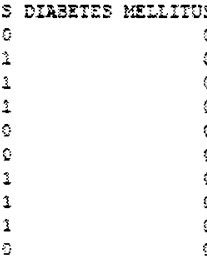

ropasor

Y Yace

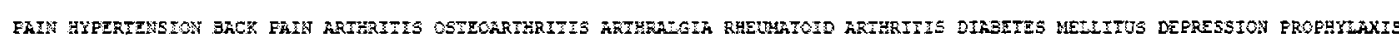

YIOWX

CIIEBEZX

Mosic

ASTERTE:

ERYERO

MOTrI

RTFROST

WETAREX

vospan

3

\begin{tabular}{|c|c|}
\hline$\overline{1}$ & \\
\hline 3 & \\
\hline$\$$ & \\
\hline$a$ & \\
\hline 8 & \\
\hline $\bar{s}$ & \\
\hline 0 & \\
\hline 1 & \\
\hline $\begin{array}{l}0 \\
\frac{1}{3}\end{array}$ & \\
\hline
\end{tabular}

0
0
0
2
0
0
0
1
0
5

2
0
1
0
0
0
2
0
0
0
1

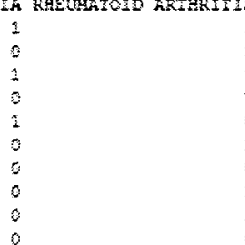

$\begin{array}{cccc}\text { IS DIREFTES MELITUS DEFRESSION FROPHYAXIS } \\ 2 & 1 & 0 & 0 \\ 1 & 0 & 0 & 0 \\ 1 & 0 & 0 & 0 \\ 2 & 0 & 0 & 0 \\ 0 & 0 & 0 & 0 \\ 1 & 2 & 0 & 0 \\ 0 & 0 & 0 & 0 \\ 1 & 0 & 0 & 0 \\ 3 & 0 & 0 & 0 \\ 0 & 1 & 0 & 1\end{array}$




\section{Appendix 2}

\section{Simulation Study}

\section{Code for signal detection}

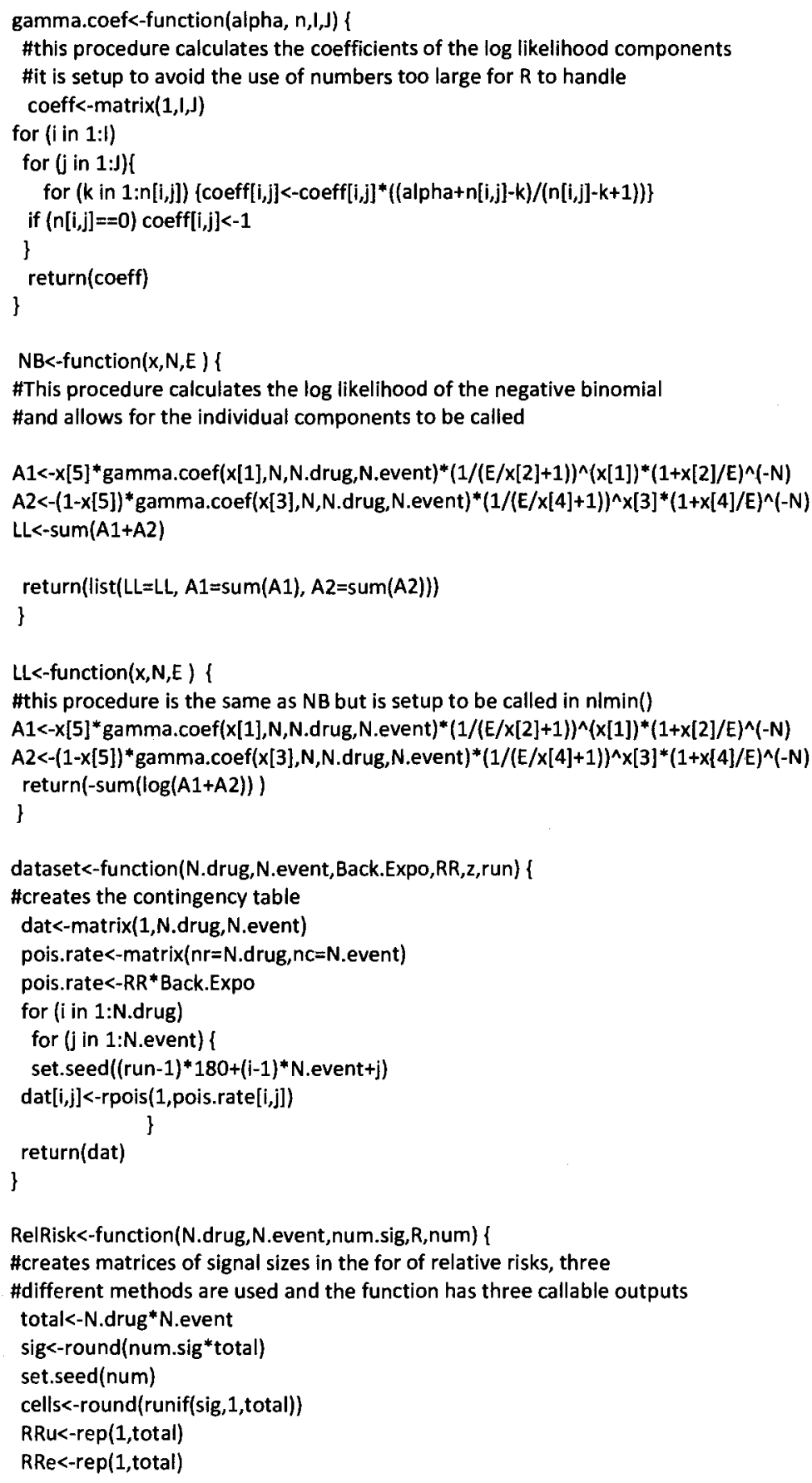




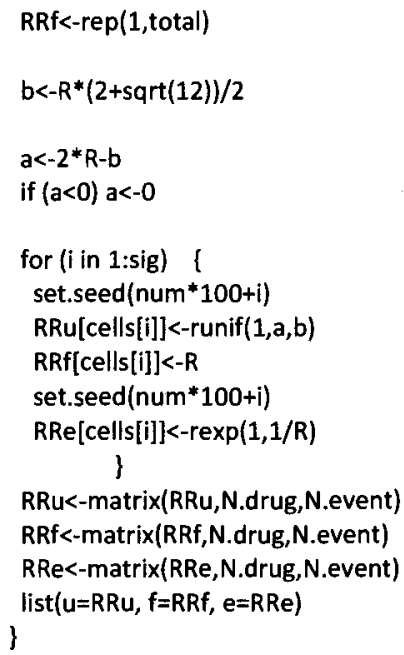

estim<-function(run,N.drug,N.event,Back.expo, RR, name.run,z) \{

\#The main function, it will call dataset() to generate new random contingency \#tables 1000 times and will assess each table for signals across all the estimators. \#The output will be a number of matrices of similar dimensions as the generated table \#and each cell value represents the number of signals flagged in that cell diverge.count $<-0$

RelR<-RR

c.PRR.cutoff $<-$ matrix $(0, n r=N$. drug, $n c=N$.event $)$

c.PRR.Cl<-matrix (0, nr=N.drug, nc= N.event) c.PRR.cutoff. $90<-$ matrix $(0, \mathrm{nr}=\mathrm{N} . \mathrm{drug}, \mathrm{nc}=\mathrm{N}$.event $)$ c.PRR.CI.90<-matrix (0, nr=N.drug, nc= N.event) c.PRR.cutoff. $99<-$ matrix $(0, n r=N . d r u g, n c=N$.event $)$ c.PRR.Cl.99<-matrix(0, nr=N.drug,nc= N.event)

c.ROR<-matrix $(0, \mathrm{nr}=\mathrm{N} . \mathrm{drug}, \mathrm{nc}=\mathrm{N}$.event $)$ c.ROR.99<-matrix (0, nr=N.drug,nc=N.event) c.ROR.90<-matrix (0, nr=N.drug, $n c=N$. event $)$

c. $Q<-$ matrix $(0, \mathrm{nr}=\mathrm{N} . \mathrm{drug}, \mathrm{nc}=\mathrm{N}$. event $)$ c. $Q .90<-$ matrix $(0, n r=N$.drug, $n c=N$.event $)$ c. $Q .99<-$ matrix $(0, n r=N . d r u g, n c=N . e v e n t)$

c.Yates $<-$ matrix $(0, n r=N$. drug, nc $=N$.event $)$ c.Yates. $99<-$ matrix $(0, n r=N . d r u g, n c=N . e v e n t)$

c. Yates.90<-matrix $(0, \mathrm{nr}=\mathrm{N} . \mathrm{drug}, \mathrm{nc}=\mathrm{N}$. event $)$

c.SPRT<-matrix (0, nr=N.drug, nc $=$ N.event $)$ c.SPRT.99<-matrix(0, nr=N.drug, nc= N.event) c.SPRT.90<-matrix $(0, \mathrm{nr}=\mathrm{N}$. drug, $\mathrm{nc}=\mathrm{N}$. event $)$

c. WHO.approx<-matrix $(0, \mathrm{nr}=\mathrm{N} . \mathrm{drug}, \mathrm{nc}=\mathrm{N}$.event $)$ c.WHO.exact<-matrix $(0, n r=N . d r u g, n c=N$.event $)$ c. WHO. approx. $.90<-$ matrix $(0, \mathrm{nr}=\mathrm{N}$. drug, $\mathrm{nc}=\mathrm{N}$.event $)$ c. WHO.exact. $90<-$ matrix $(0, \mathrm{nr}=\mathrm{N} . \mathrm{drug}, \mathrm{nc}=\mathrm{N}$.event $)$ c. WHO.approx. $99<-$ matrix $(0, \mathrm{nr}=\mathrm{N}$.drug, $\mathrm{nc}=\mathrm{N}$.event $)$ c.WHO.exact. $99<-$ matrix $(0, n r=N . d r u g, n c=N$.event $)$

c.EB.roux $<-$ matrix $(0, n r=N . d r u g, n c=N$.event $)$ c.EB.dist $<-$ matrix $(0, n r=N . d r u g, n c=N$.event $)$ c.EB.dist.99<-matrix (0, nr=N.drug,nc=N.event) 


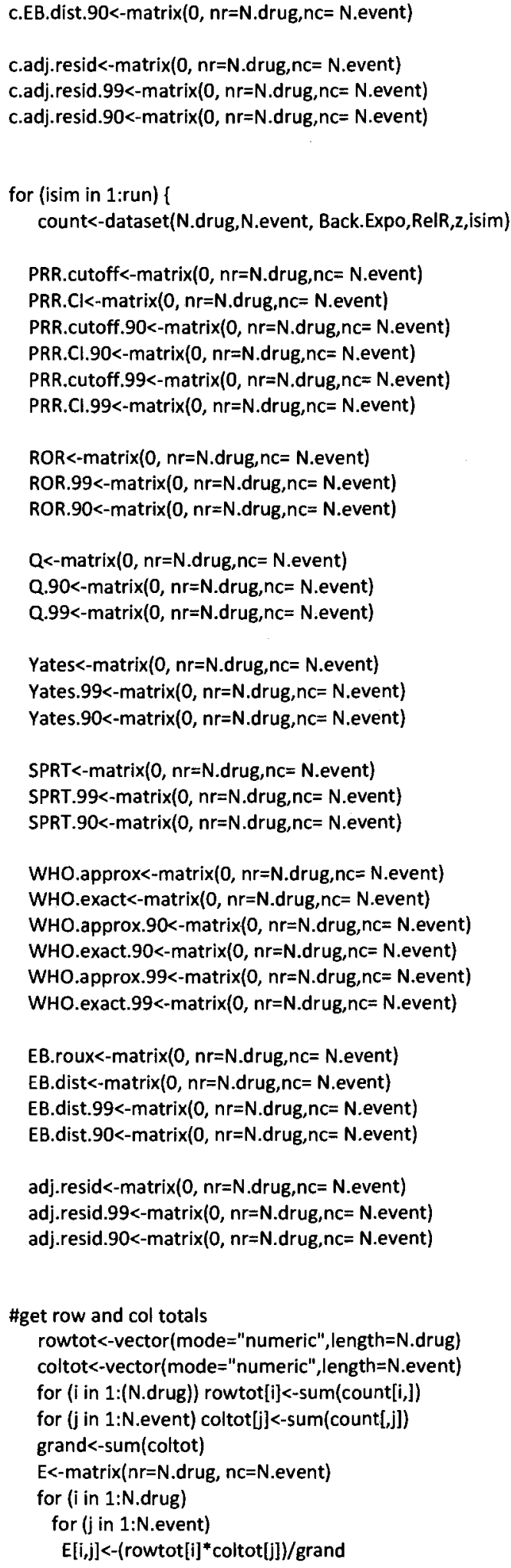

EB.roux $<-$ matrix $(0, n r=N . d r u g, n c=N$. event $)$ EB.dist $<-$ matrix $(0, n r=N$. drug, $n c=N$.event $)$ EB.dist. $99<-$ matrix $(0, n r=N$.drug, nc $=N$.event $)$ EB.dist. $90<-$ matrix $(0, n r=N . d r u g, n c=N$.event $)$

adj.resid<-matrix (0, $\mathrm{nr}=\mathrm{N} . \mathrm{drug}, \mathrm{nc}=\mathrm{N}$. event $)$ adj.resid.99<-matrix (0, nr=N.drug,nc= N.event) adj.resid.90<-matrix $(0, n r=N . d r u g, n c=N . e v e n t)$

\section{\#get row and col totals}

rowtot $<-$ vector(mode $=$ "numeric", length=N.drug) coltot $<$-vector(mode $=$ "numeric", length $=N$.event) for (i in 1:(N.drug)) rowtot[i]<-sum(count $[i]$, for ( $j$ in 1:N.event) coltot $[j]<-s u m($ count $[j]$ ) grand<-sum(coltot)

$\mathrm{E}<-$ matrix(nr=N.drug, nc=N.event) for (i in 1:N.drug)

for ( $\mathrm{j}$ in 1:N.event)

$E[i, j]<-\left(\operatorname{rowtot}[i]^{*}\right.$ coltot $\left.[j]\right) /$ grand 


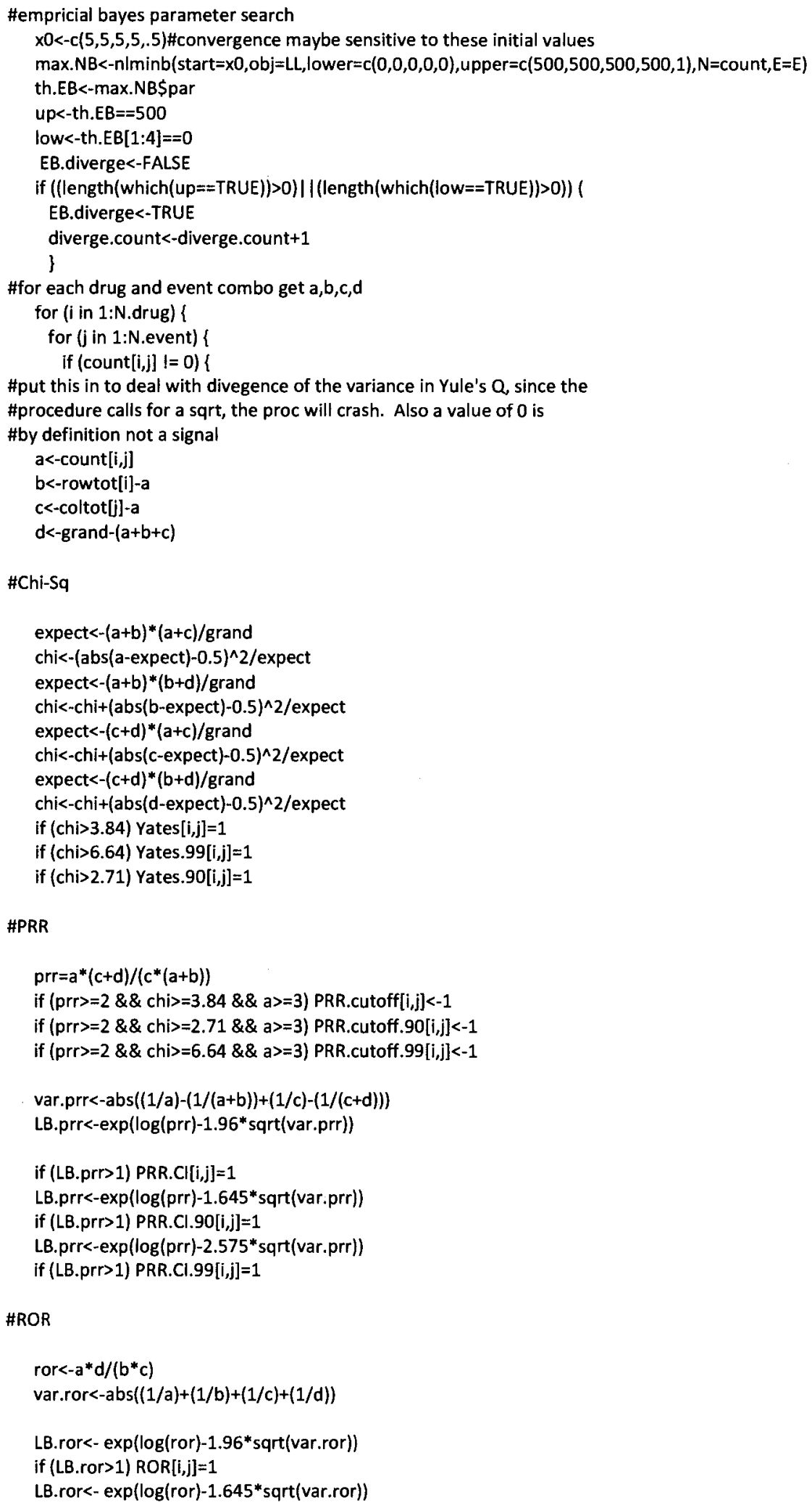


if (LB.ror>1) ROR.90[i,j] $=1$

LB.ror<- exp(log(ror)-2.575*sqrt(var.ror))

if (LB.ror>1) ROR.99[i,j] $=1$

\section{\#Yule's Q}

Yule. $Q<-($ ror-1)/(ror+1)

LB.Yule.Q<-Yule.Q-1.96*0.5*(1-Yule. Q^2)*sqrt(var.ror)

if (LB.Yule. $Q>0) Q[i, j]=1$

LB.Yule.Q<-Yule.Q-1.645*0.5*(1-Yule.Q^2)* sqrt(var.ror)

if (LB.Yule. $Q>0)$ Q $Q .90[i, j]=1$

LB.Yule.Q<-Yule.Q-2.575*0.5*(1-Yule.Q^2)*sqrt(var.ror)

if (LB.Yule.Q>0) $Q .99[i, j]=1$

\#SPRT wth an $2 R R$ (event) $=R R$ (coincidental) and type1=type $2=0.05$

\#2.94=In((1-beta)/alpha))

if $\left(\left(a^{*} \log (2)-E[i, j]\right)>=2.94\right) \operatorname{SPRT}[i, j]=1$

if $\left(\left(\mathrm{a}^{*} \log (2)-E[i, j]\right)>=2.197\right) S P R T .90[i, j]=1 \quad \# a=.1, B=.1$

if $\left(\left(a^{*} \log (2)-E[i, j]\right)>=4.59512\right)$ SPRT.99[i,j] $]=1 \quad \# a=.01, \mathrm{~B}=.01$

\#WHO method

p1<-1+a+b

p $2<-1+d-a-b$

$q 1<-1+a+c$

$q 2<-1+d-a-c$

$r 1<-1+a$

$r 2<-3+d-a$

\#approx

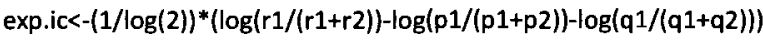

var.ic $<-a b s\left((1 /(2 * \log (2)))^{*}\left(p 2 /(p 1 *(p 1+p 2+1))+q 2 /\left(q 1^{*}(q 1+q 2+1)\right)+r 2 /(r 1 *(r 1+r 2+1))\right)\right)$

if ((exp.ic-1.96*sqrt(var.ic))>0) WHO.approx[i,j] $]=1$

if ((exp.ic-1.645*sqrt(var.ic))>0) WHO.approx.90[i,j]=1

if ((exp.ic-2.575*sqrt(var.ic) )>0) WHO.approx.99[i,j] $=1$

\#exact

exp.exact.ic $<-(1 / \log (2))^{*}($ digamma(r1)-digamma(r1+r2)-(digamma(p1)-digamma(p1+p2)+digamma(q1)-digamma(q1+q2)))

var.exact.ic<-abs $\left(\left(1 /\left(2^{*} \log (2)\right)\right)^{*}(\right.$ trigamma(r1)-trigamma(r1+r2)-(trigamma(p1)-trigamma(p1+p2)+trigamma(q1)-

trigamma $(q 1+q 2))))$

if ((exp.exact.ic-1.96*sqrt(var.exact.ic))>0) WHO.exact $[1, j]=1$

if $(($ exp.exact.ic-1.645*sqrt(var.exact.ic) $)>0)$ WHO.exact. $90[i, j]=1$

if $(($ exp.exact.ic-2.575*sqrt(var.exact.ic $))>0)$ WHO.exact. $99[i, j]=1$

if (EB.diverge==FALSE)

\#empirical bayes

negbin<-NB(th.EB,count,E)

Qn<-negbin\$A1/negbin\$LL

W1<-digamma(th.EB[1]+a)-log(th.EB[2]+E[i,j])

W2<-digamma(th.EB[3]+a)- $\log ($ th. EB $[4]+E[i, j])$

Ex.EBGM<-(1/log(2))*(Qn*W1+(1-Qn)*W2)

crit<-th. $E B[5]^{*}$ qgamma(0.025, shape=th.EB[1]+a, scale $=(1 /(E[i, j]+$ th. $\left.E B[2]))\right)+(1-\text { th. } E B[5])^{*} q g a m m a(0.025$, shape $=$ th. $E B[3]+a$, scale $=(1 /(E[i, j]+$ th. EB $[4])))$

if (crit>1) EB.dist $[i, j]<-1$

crit<-th.EB[5]* $q g a m m a(0.05$, shape $=$ th. $E B[1]+a$, scale $=(1 /(E[i, j]+$ th. $E B[2])))+(1-\text { th. } E B[5])^{*} q g a m m a(0.05$, shape $=$ th. $E B[3]+a$, scale $=(1 /(E[i, j]+$ th. $E B[4])))$

if (crit>1) EB.dist.90[i,j]<-1 
crit<-th.EB[5]*qgamma $(0.005$, shape=th. $E B[1]+a$, scale $=(1 /(E[i, j]+t h . E B[2]))+(1-$ th. $E B[5]) *$ qgamma $(0.005$, shape $=$ th. EB $[3]+a$, scale $=(1 /(E[i, j]+$ th. $E B[4])))$

if (crit>1) EB.dist.99[i,j]<-1

\#Roux criteria
delta1<-0
delta2<-0
if $((($ th. EB $[1]+\mathrm{a}) /($ th. EB $[2]+E[i, j]))>1)$ delta $1<-1$
if $((($ th. EB $[3]+\mathrm{a}) /($ th. EB $[4]+E[i, j]))>1)$ delta2<-1

EBp $<-Q n *$ delta1+(1-Qn)*delta2

if $(\mathrm{EBp}>0.5) \mathrm{EB}$.roux $[i, j]=1$

\}

\#Adjusted resid

rij<-(a-E[i,j])/sqrt(E[i,j]*(1-rowtot[i]/grand)*(1-coltot[j]/grand))

if (rij>1.96) adj.resid[i,j]=1

if (rij>1.645) adj.resid.90[i,j]=1

if (rij>2.575) adj.resid.99[i,j] $=1$

)

\}

1

c.PRR.cutoff $<-c . P R R . c u t o f f+P R R . c u t o f f$

c.PRR.Cl<-c.PRR.CI+PRR.CI

c.PRR.cutoff.99<-c.PRR.cutoff.99+PRR.cutoff.99

c.PRR.Cl.99<-c.PRR.Cl.99+PRR.Cl.99

c.PRR.cutoff. $90<-$ - .PRR.cutoff. $90+$ PRR.cutoff. 90

c.PRR.Cl.90<-c.PRR.Cl.90+PRR.Cl.90

C. $R O R<-C . R O R+R O R$

C.ROR.99<-C.ROR.99+ROR.99

C.ROR. $90<-$ C.ROR. $90+$ ROR. 90

c. $Q<-c . Q+Q$

c. Q. $90<-C . Q .90+Q .90$

c. Q. $99<-c . Q .99+Q .99$

c.Yates<-c.Yates+Yates

c.Yates.99<-c.Yates.99+Yates.99

c.Yates.90<-c.Yates.90+Yates.90

c.SPRT <-c.SPRT+SPRT

C.SPRT.99<-c.SPRT.99+SPRT.99

c.SPRT.90<-c.SPRT.90+SPRT.90

c. WHO.approx<-c. WHO.approx+WHO.approx

c. WHO.exact<-c. WHO.exact+WHO.exact

c.WHO.approx. $99<-c$. WHO.approx.99+WHO.approx.99

c.WHO.exact.99<-c.WHO.exact.99+WHO.exact.99

c.WHO.approx. $90<-$ - WHO.approx. $90+$ WHO.approx. 90

c.WHO.exact. $90<-c$. WHO.exact. $90+$ WHO.exact. 90

c.EB.roux $<-c$.EB.roux+EB.roux

c.EB.dist<-c.EB.dist+EB.dist

c.EB.dist. $90<-$ c.EB.dist. $90+$ EB.dist. 90 


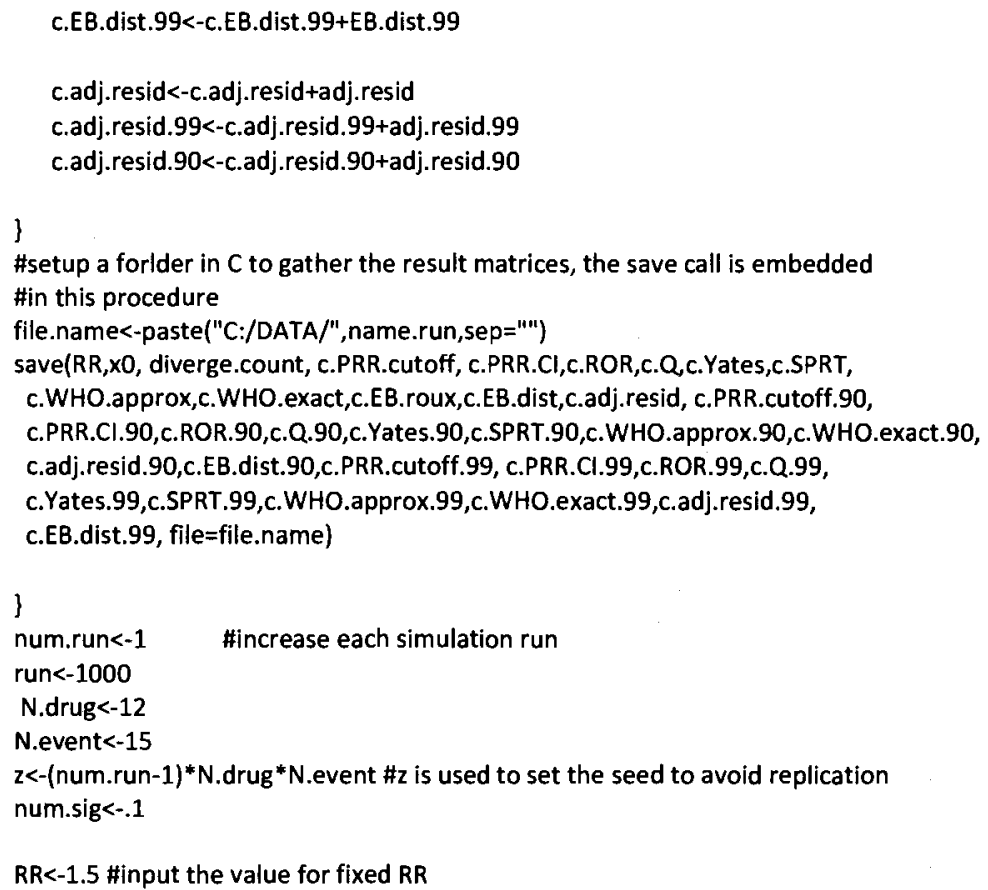

$R R<-1.5$ \#input the value for fixed RR

set.seed(num.run)

Backmatrix<-matrix(runif(N.event,.00002,.00007))

Expo<-seq $(200000$, by $=10000$,length $=N$. drug $)$

Back.Expo<-Expo\%*\%t(Backmatrix)

RR<-RelRisk(N.drug,N.event, num.sig,RR, num.run)

fi.title<-as.character(num.run)

un.title<-paste(fi.title,".un",sep="")

ex.title<-paste(fi.title,".ex",sep="")

estim(run,N.drug,N.event,Back.expo,RR\$̦f,fi.title,z)

estim(run,N.drug,N.event,Back.expo, RR\$̧u, un.title,z)

estim(run,N.drug,N.event,Back.expo,RR\$e,ex.title,z)

Function to generate Power coordinates

powercoor $<$-function(sig,RR,div) \{

signals<-which $(R R>1)$

RR.X $x<-\{\}$

Pow. $Y<-\{\}$

for (j in 1:length(signals)) Pow.Y[j]<-sig[signals[j]]/(1000-div)

for ( $j$ in 1:length(signals)) RR.X[j]<-RR[signals[j]]

Pow.Coor<-rbind(RR.X,Pow.Y)

return(t(Pow.Coor)) 


\section{Power Curves for Simulation}

Generated from a Fixed RR
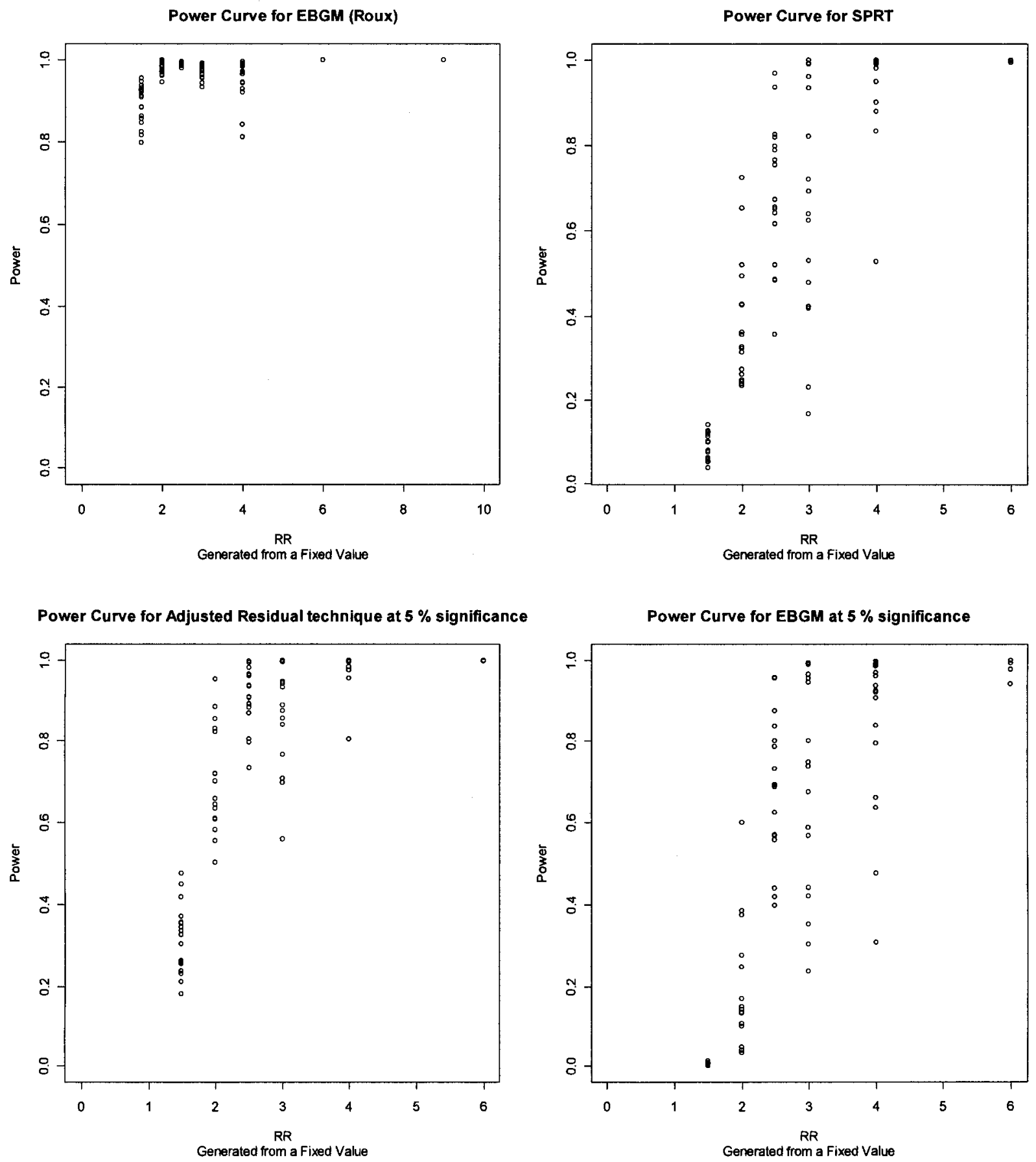

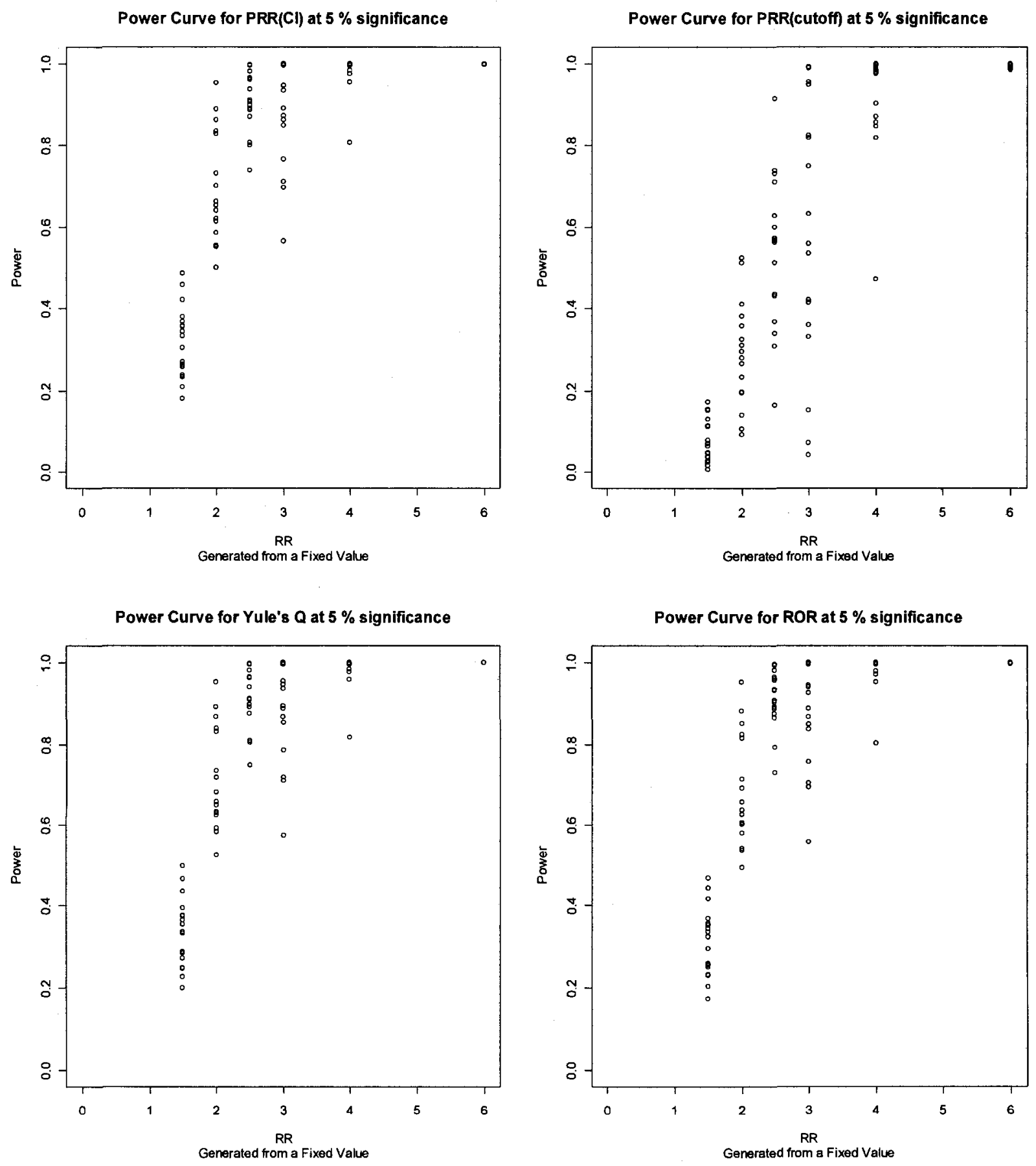

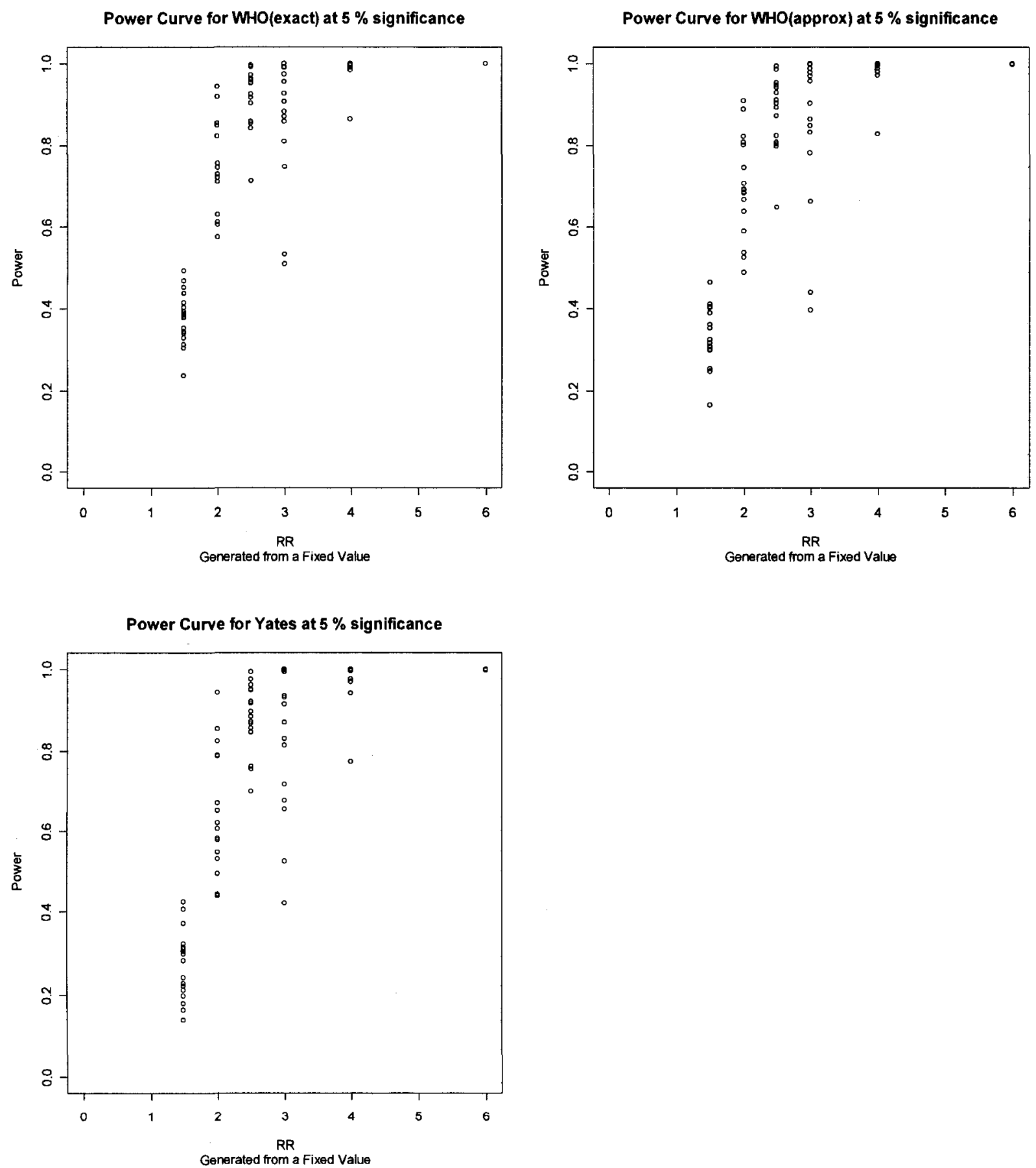

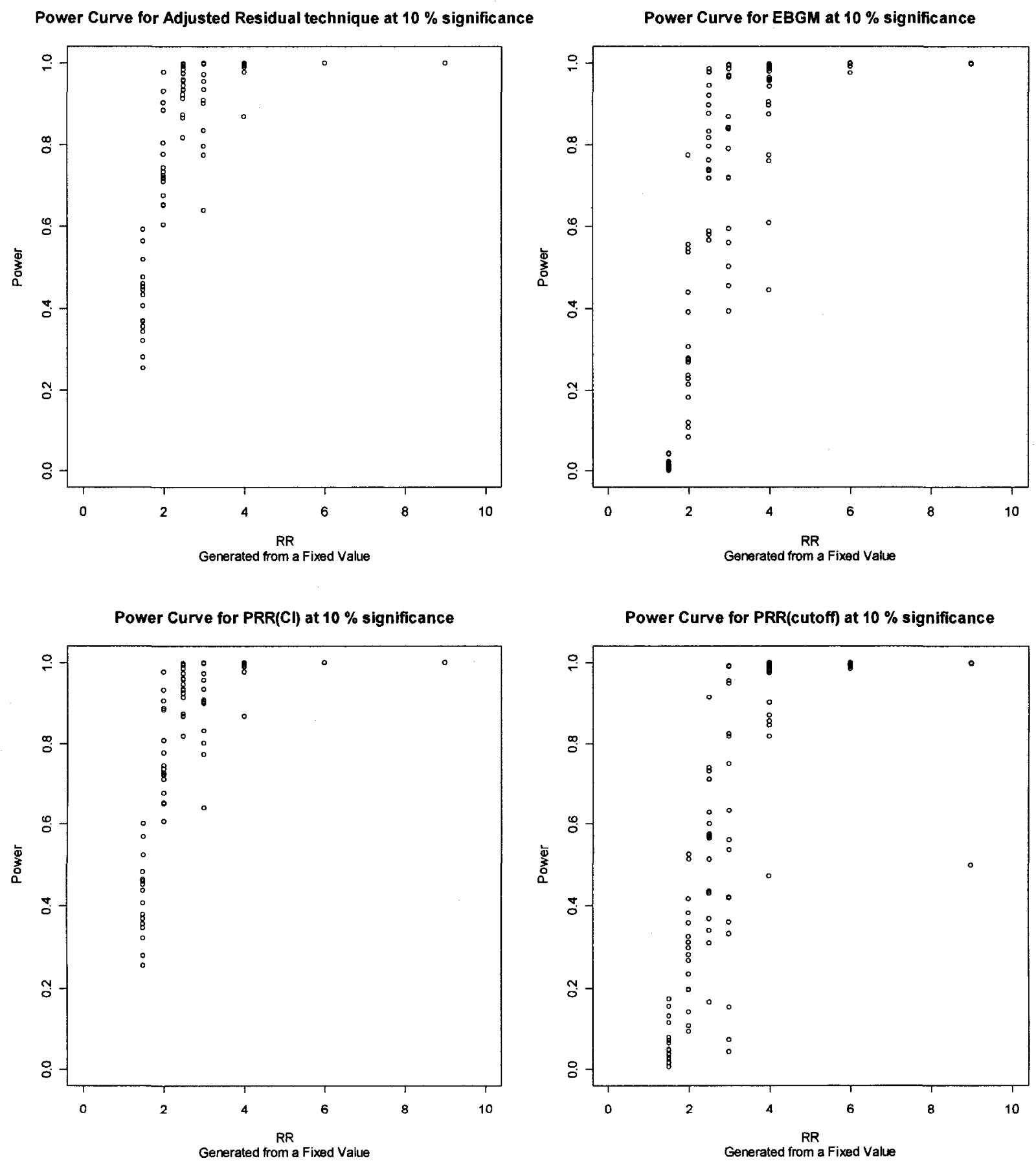


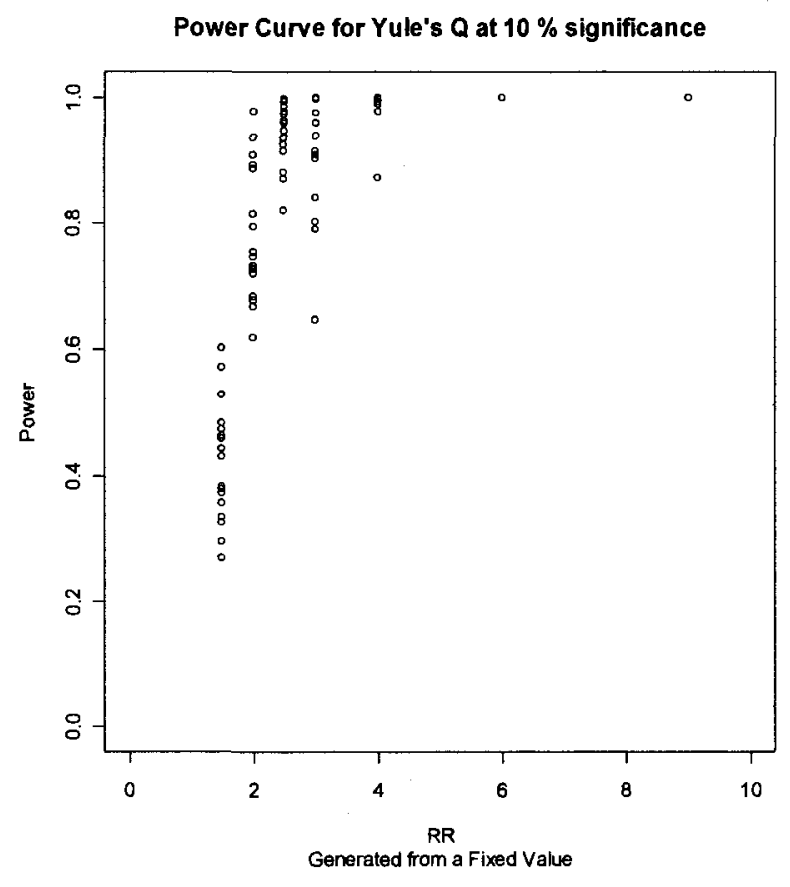

Power Curve for WHO(exact) at $10 \%$ significance

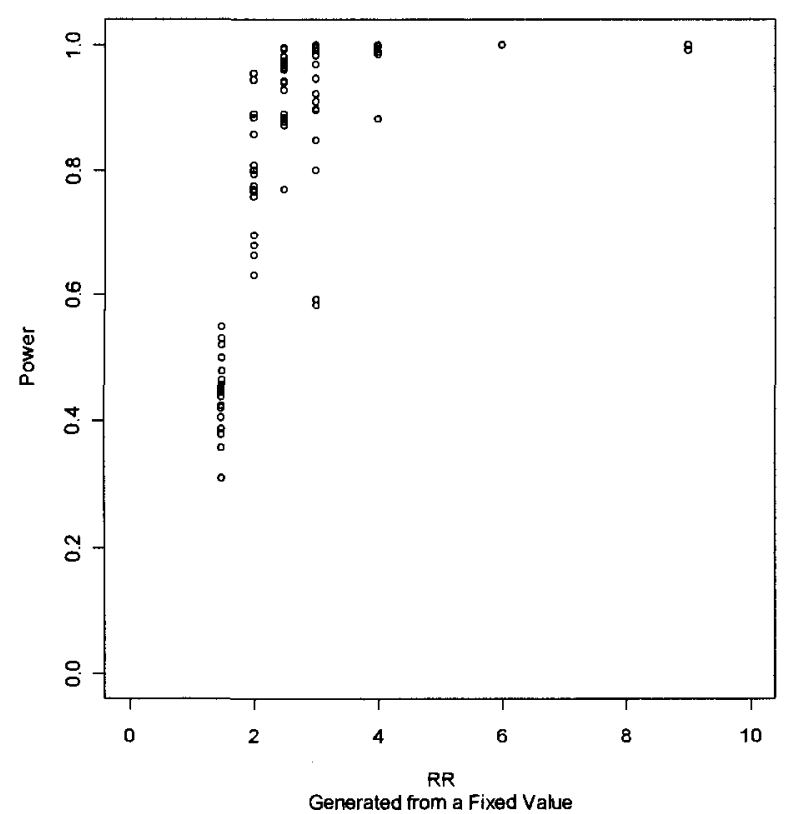

Power Curve for ROR at $10 \%$ significance

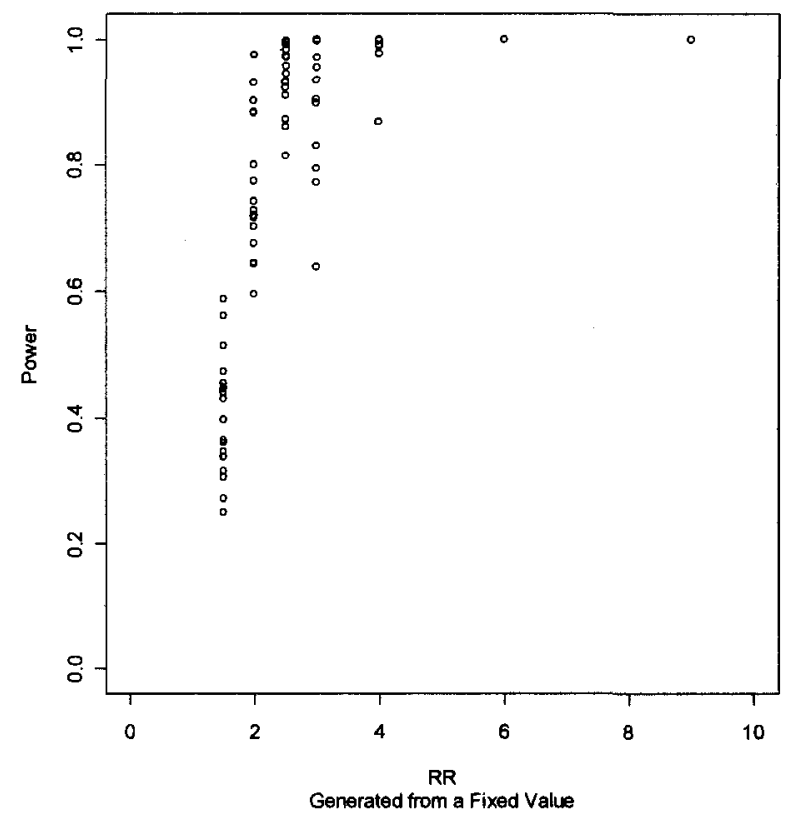

Power Curve for WHO(approx) at $10 \%$ significance

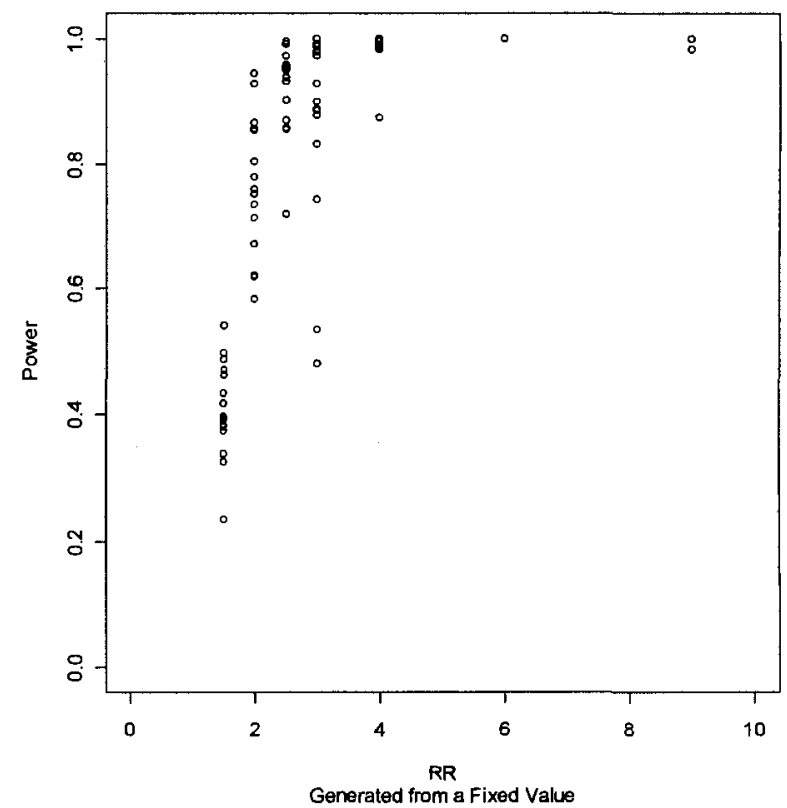


Power Curve for Yates at $10 \%$ significance

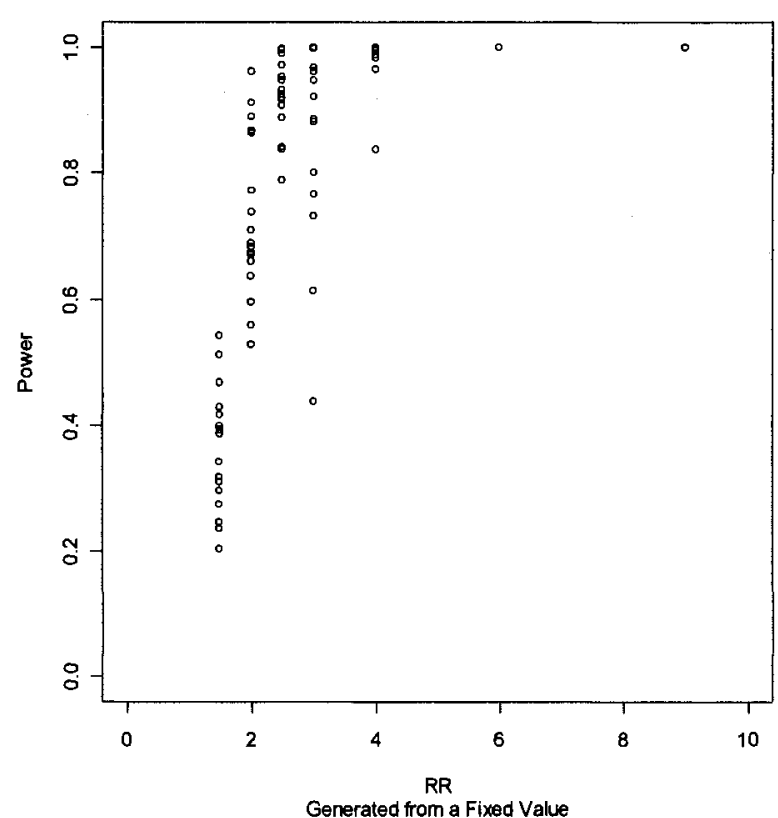

Generated from a Fixed Value 

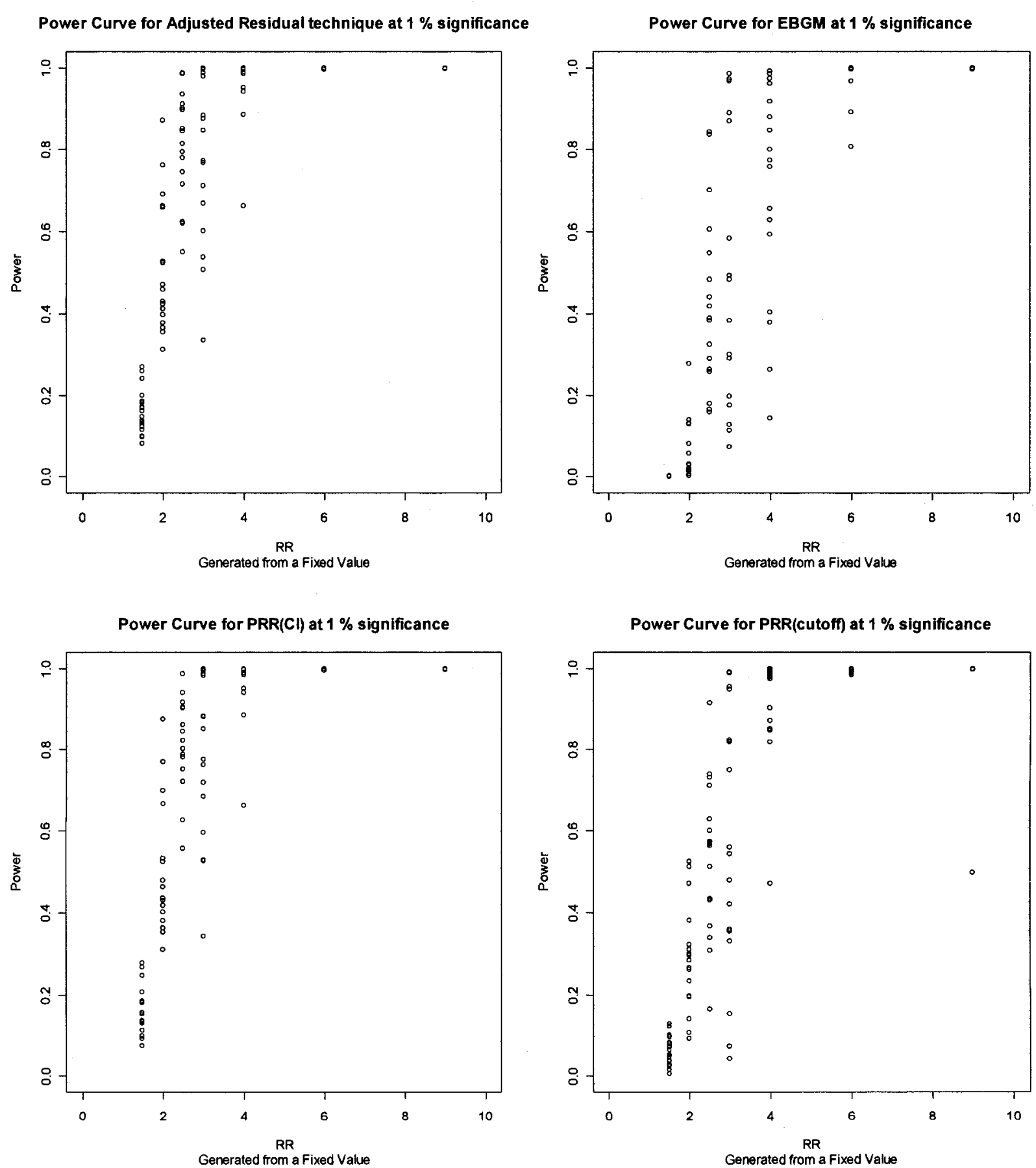

Generated from a Fixed Value 

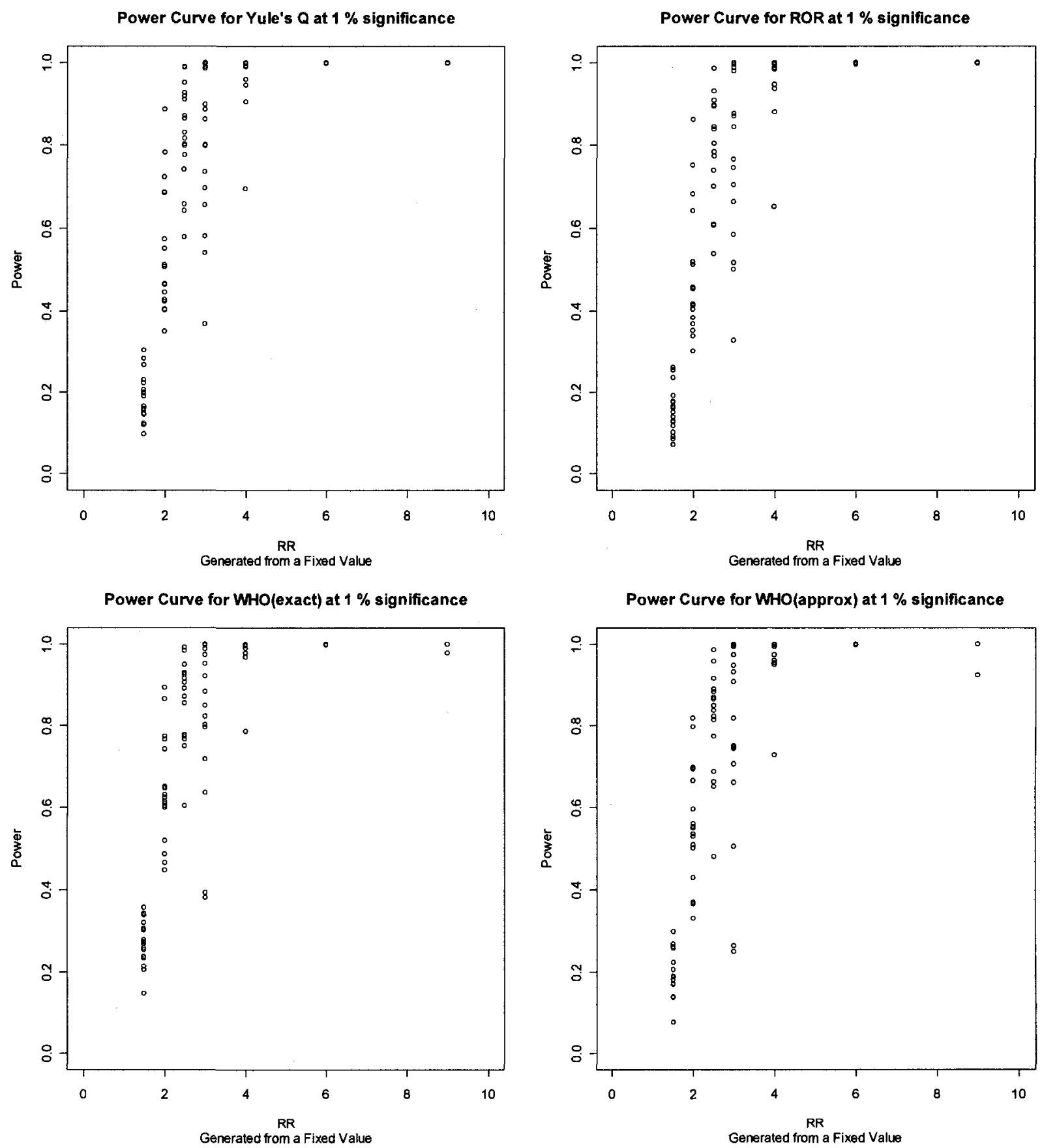
Power Curve for Yates at $1 \%$ significance

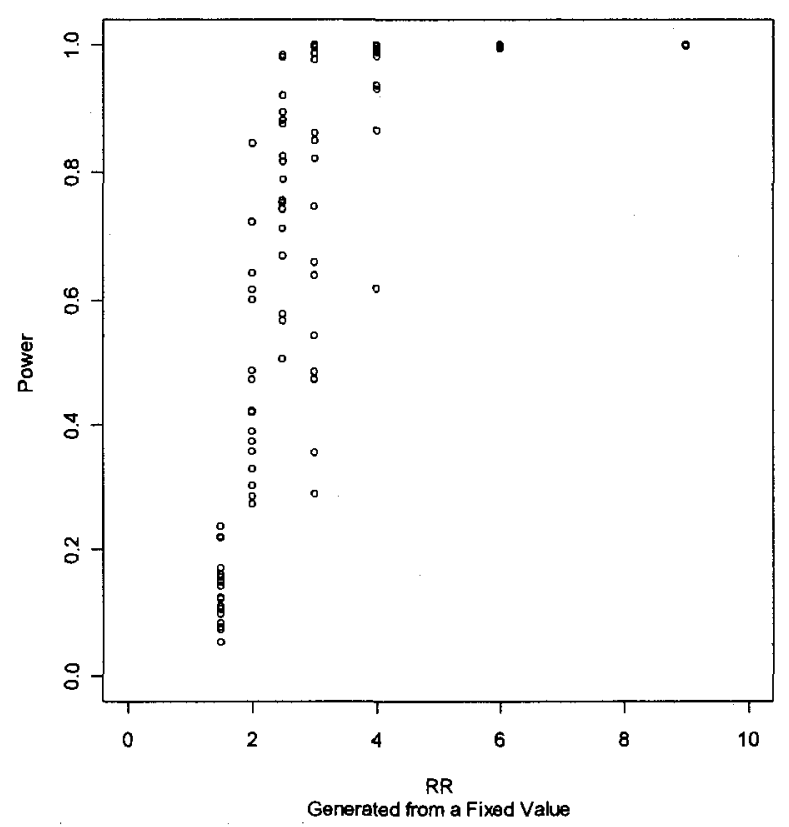


Generated from a Uniform distribution
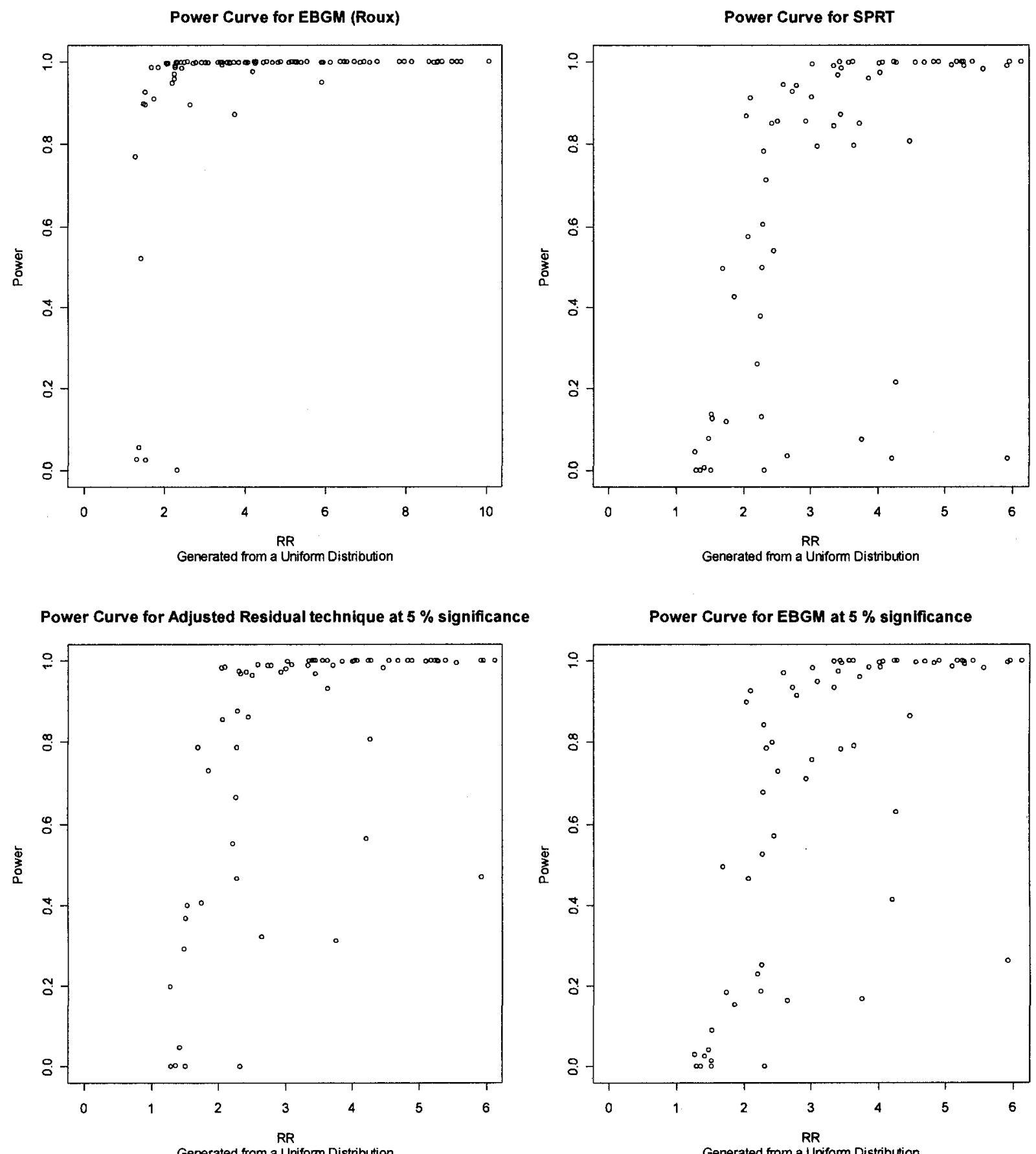

Generated from a Uniform Distribution Generated from a Uniform Distribution 

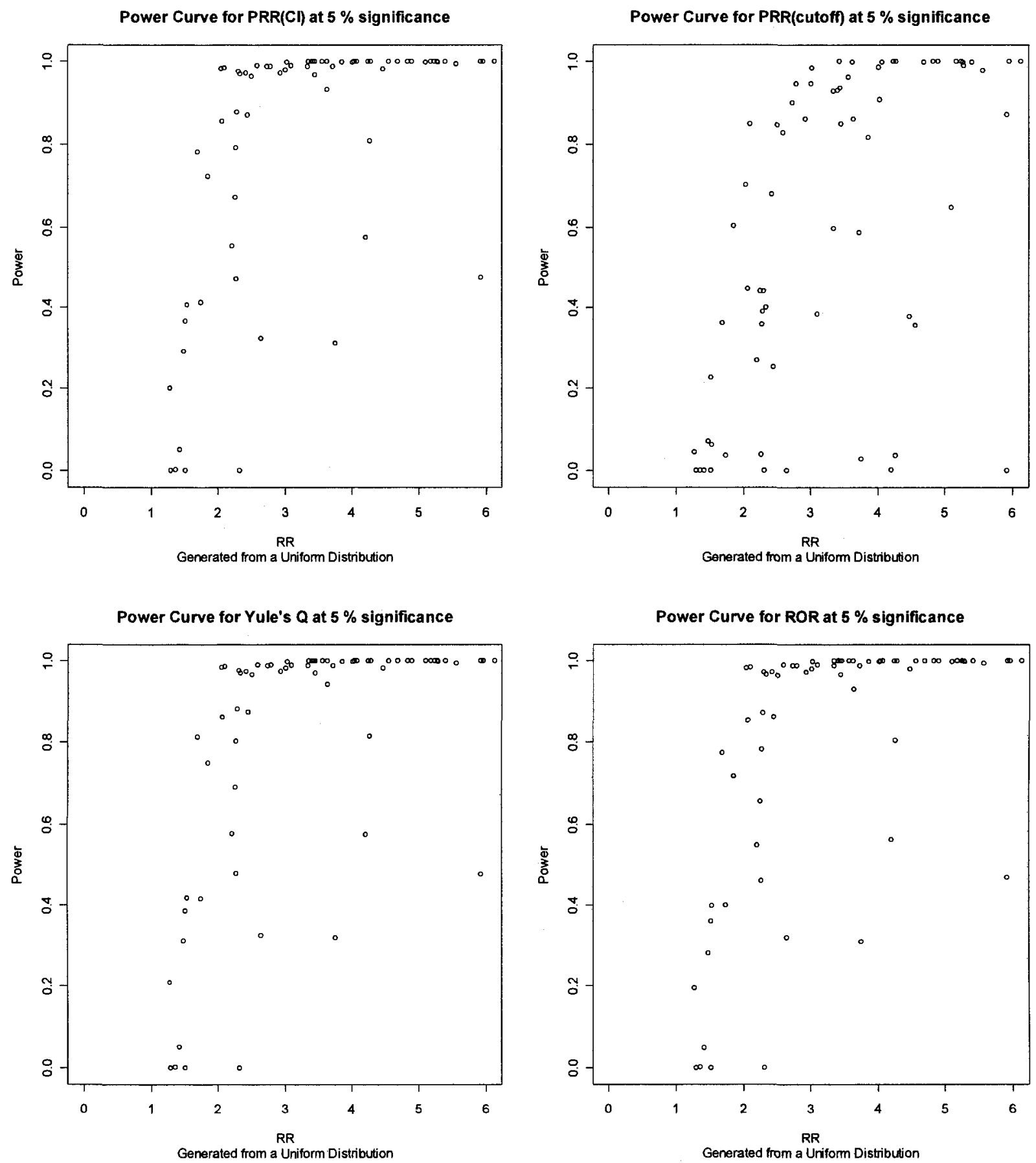
Power Curve for WHO(exact) at $5 \%$ significance

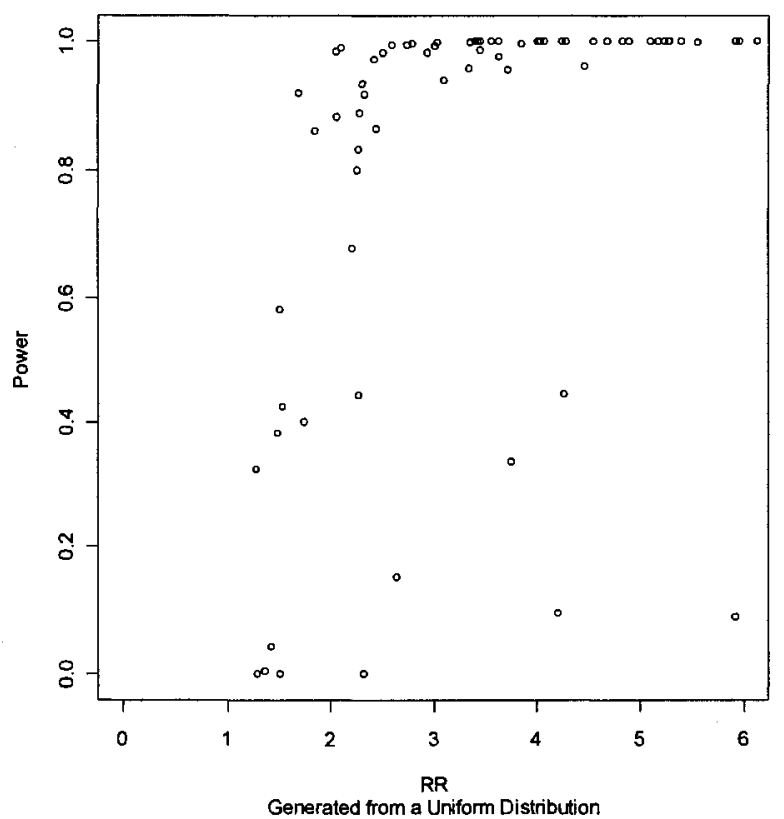

Power Curve for Yates at $5 \%$ significance

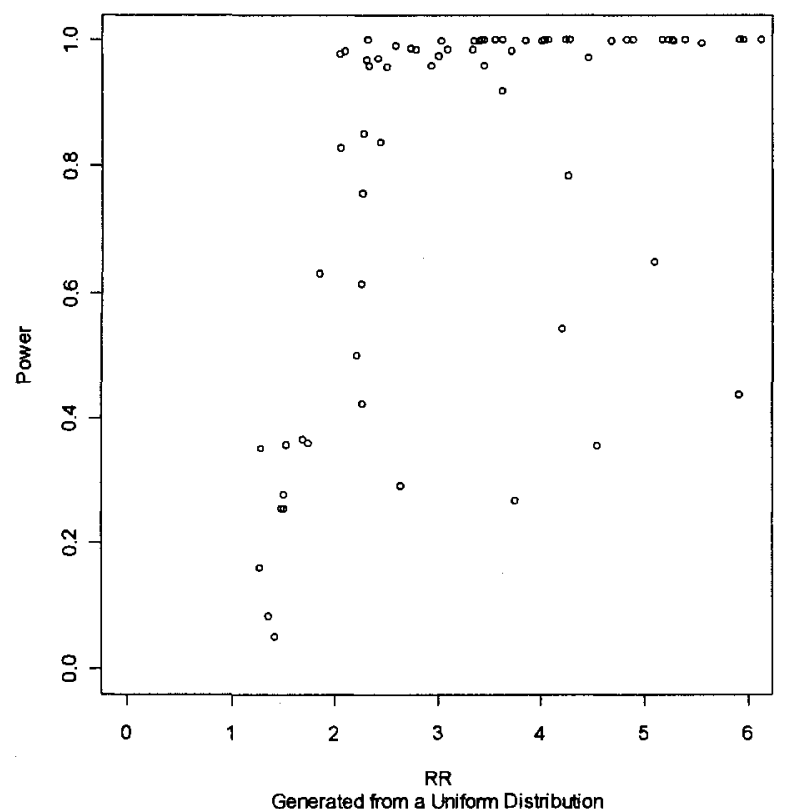

Power Curve for WHO(approx) at $5 \%$ significance

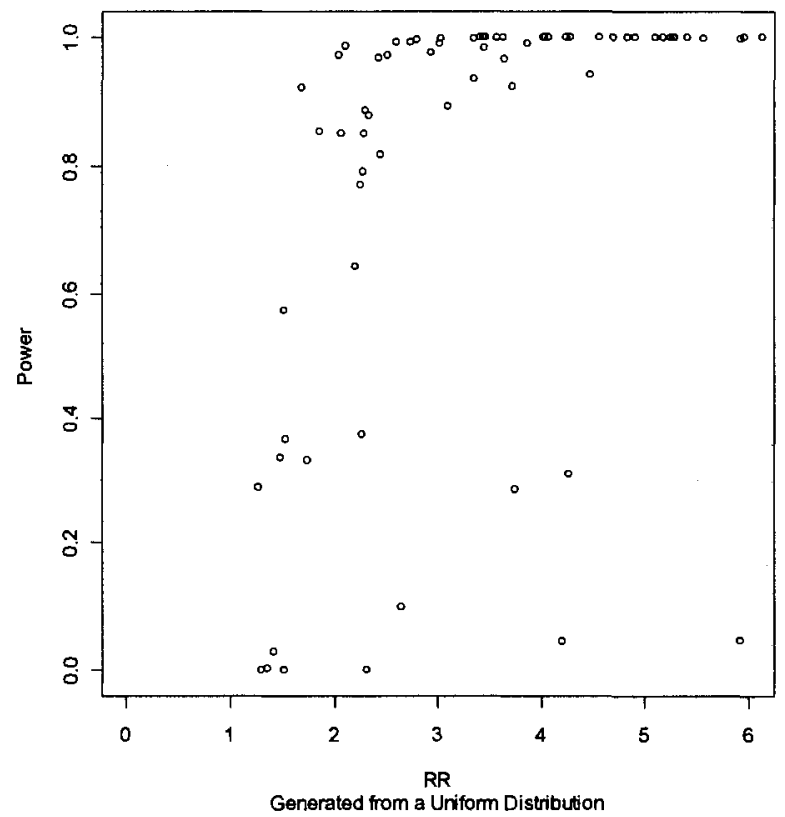



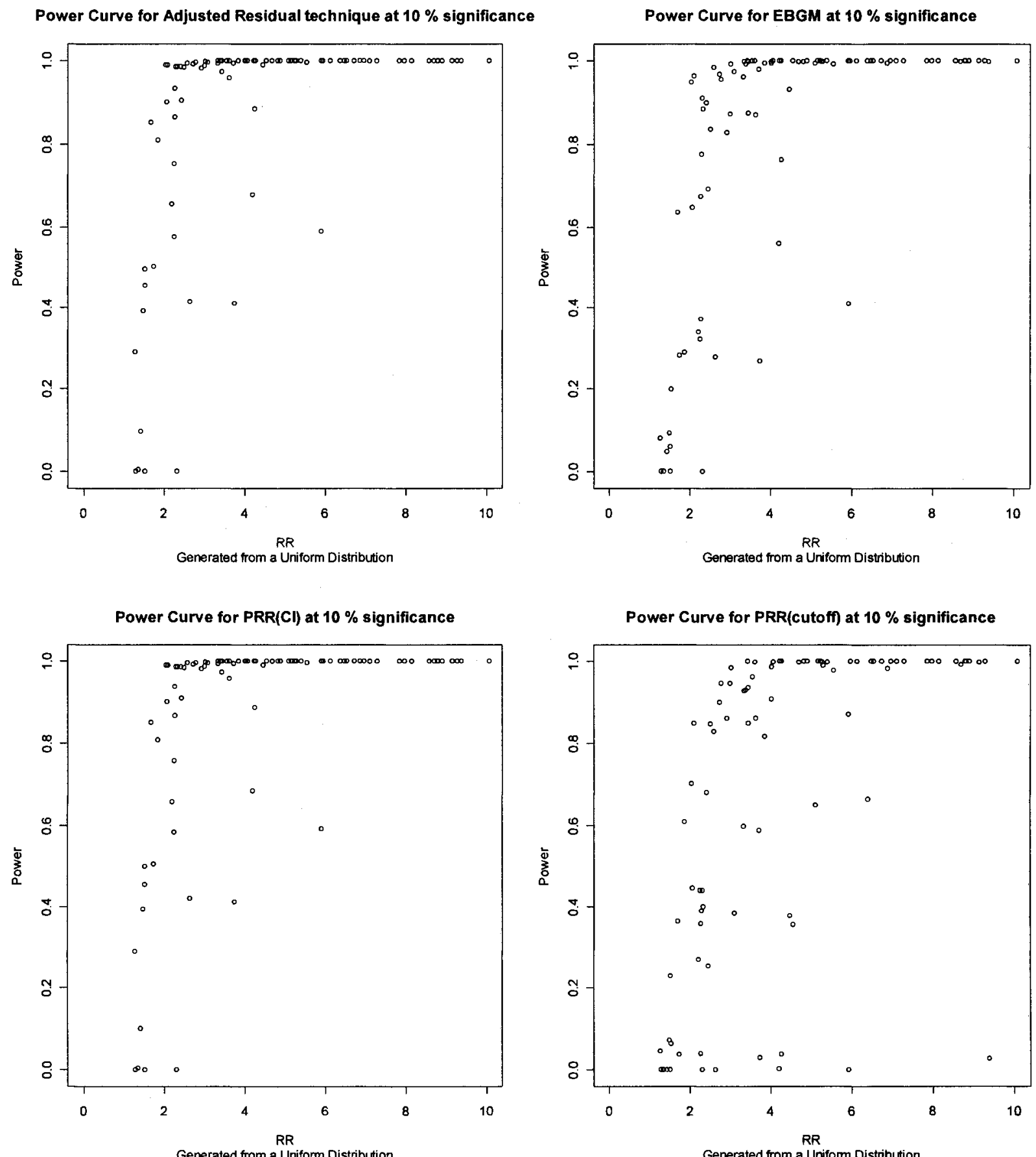

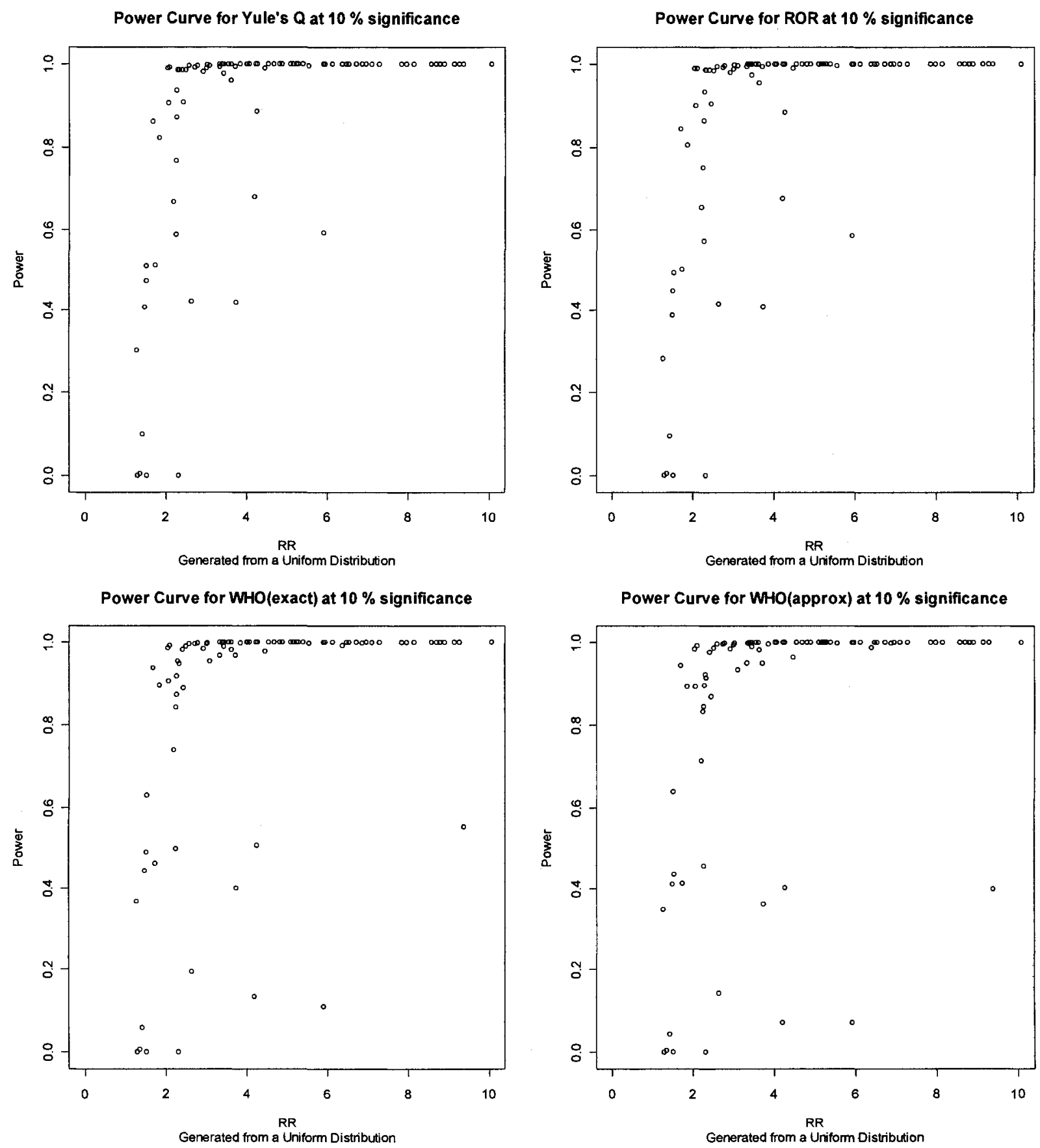


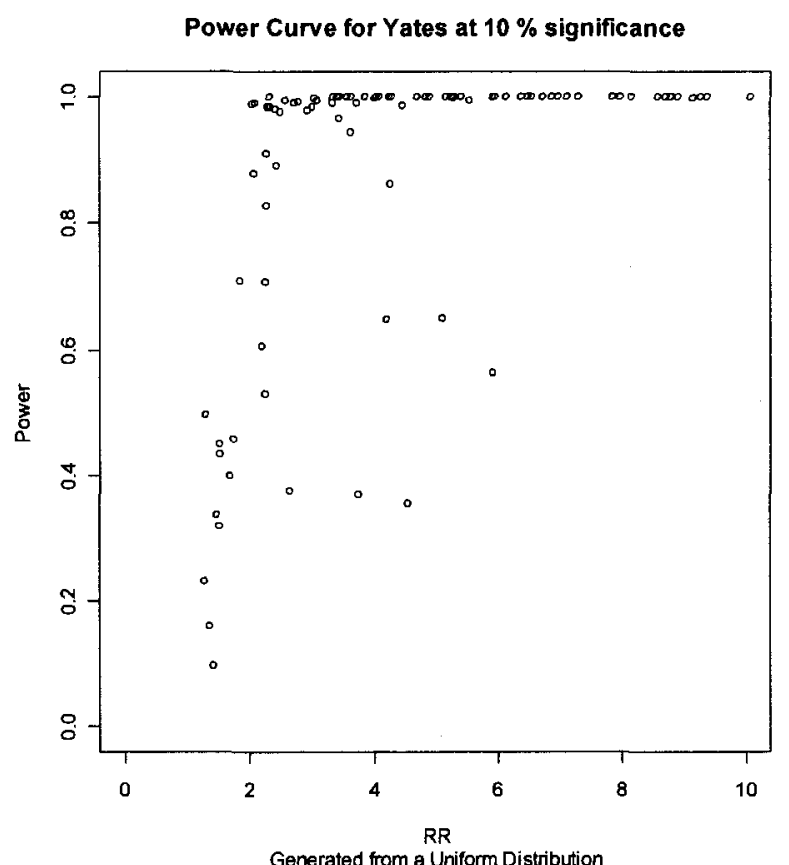



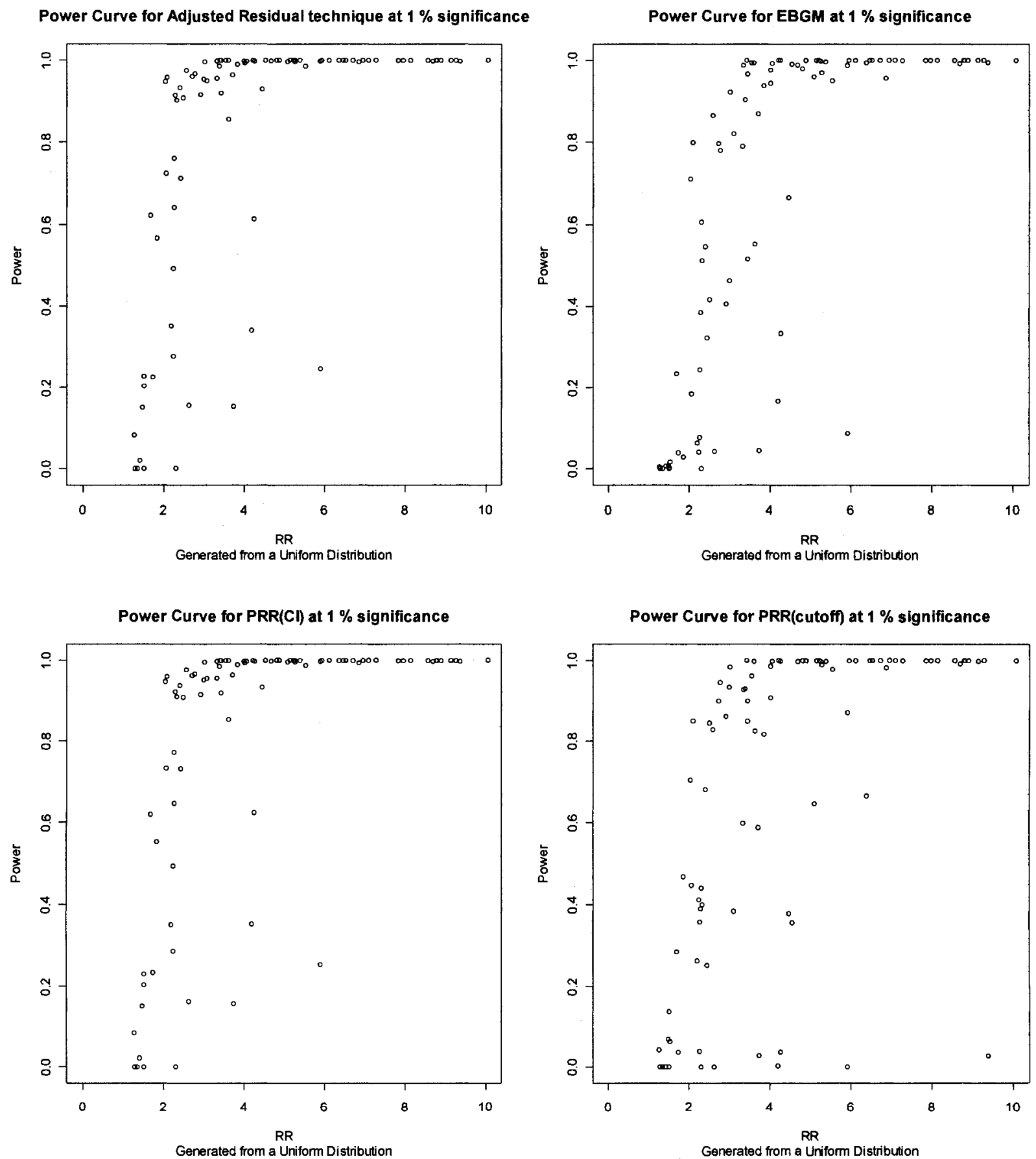

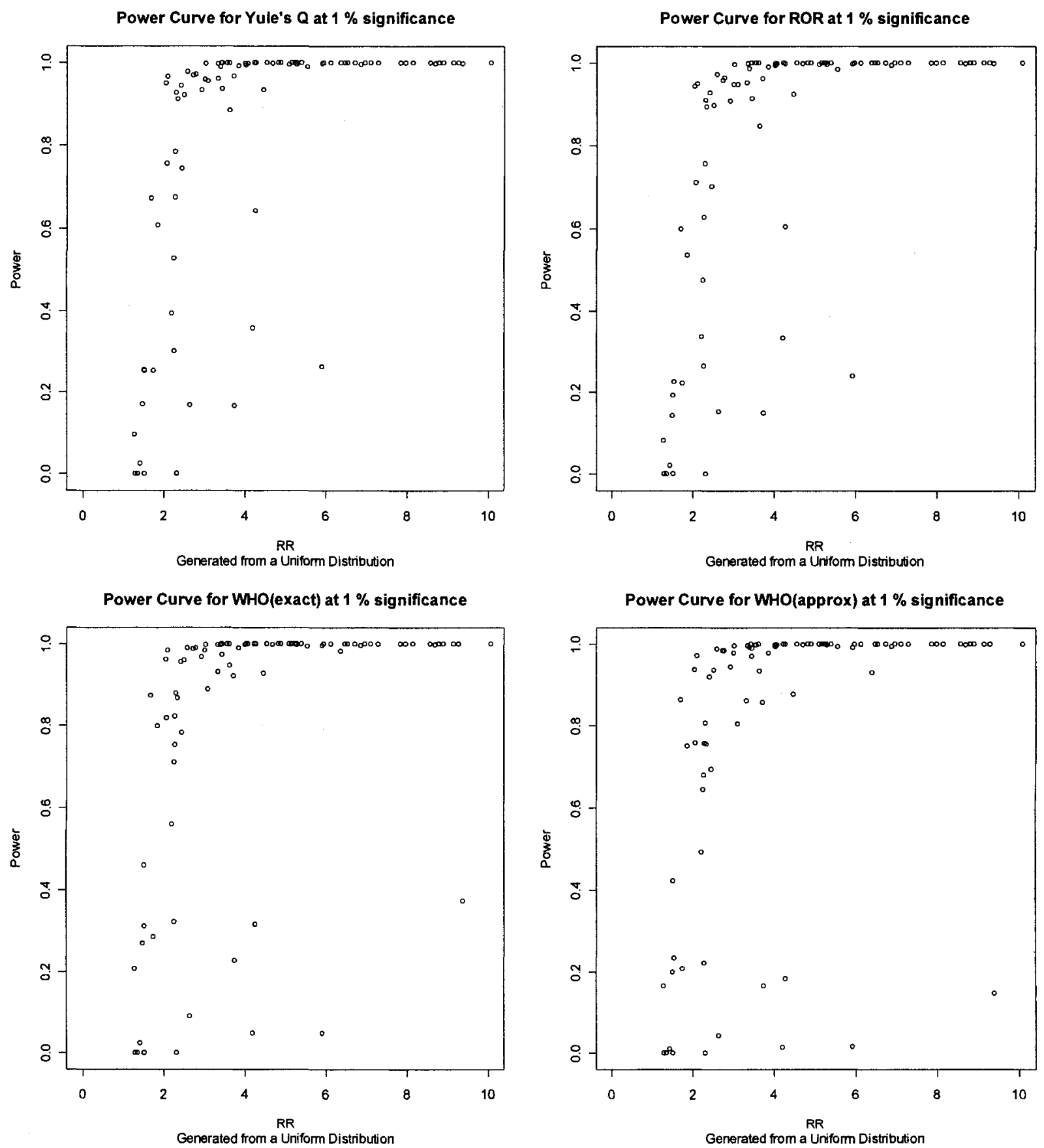
Power Curve for Yates at $1 \%$ significance

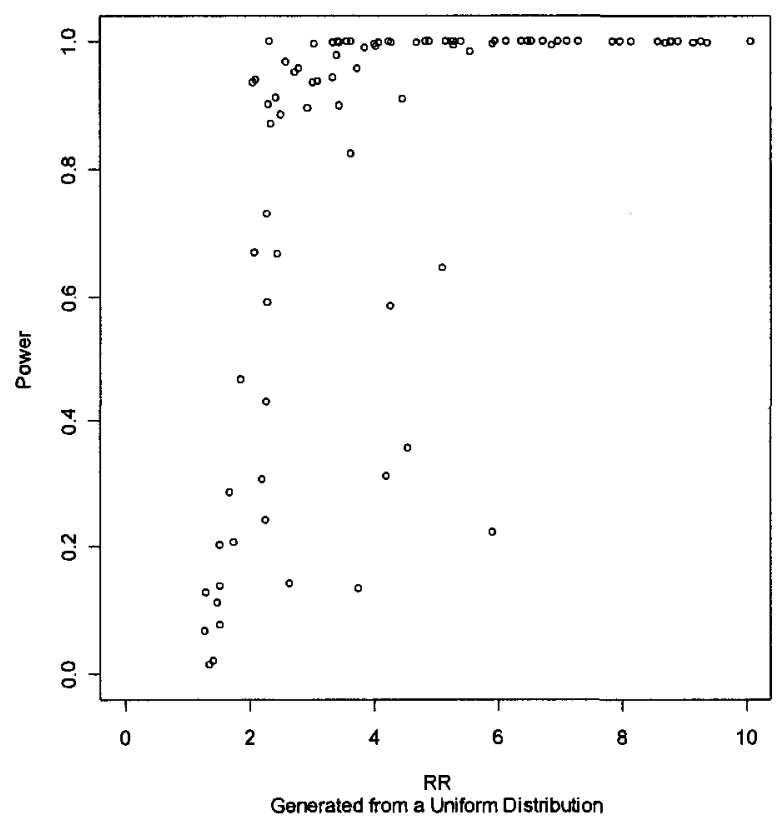


Generated from an Exponential Distribution
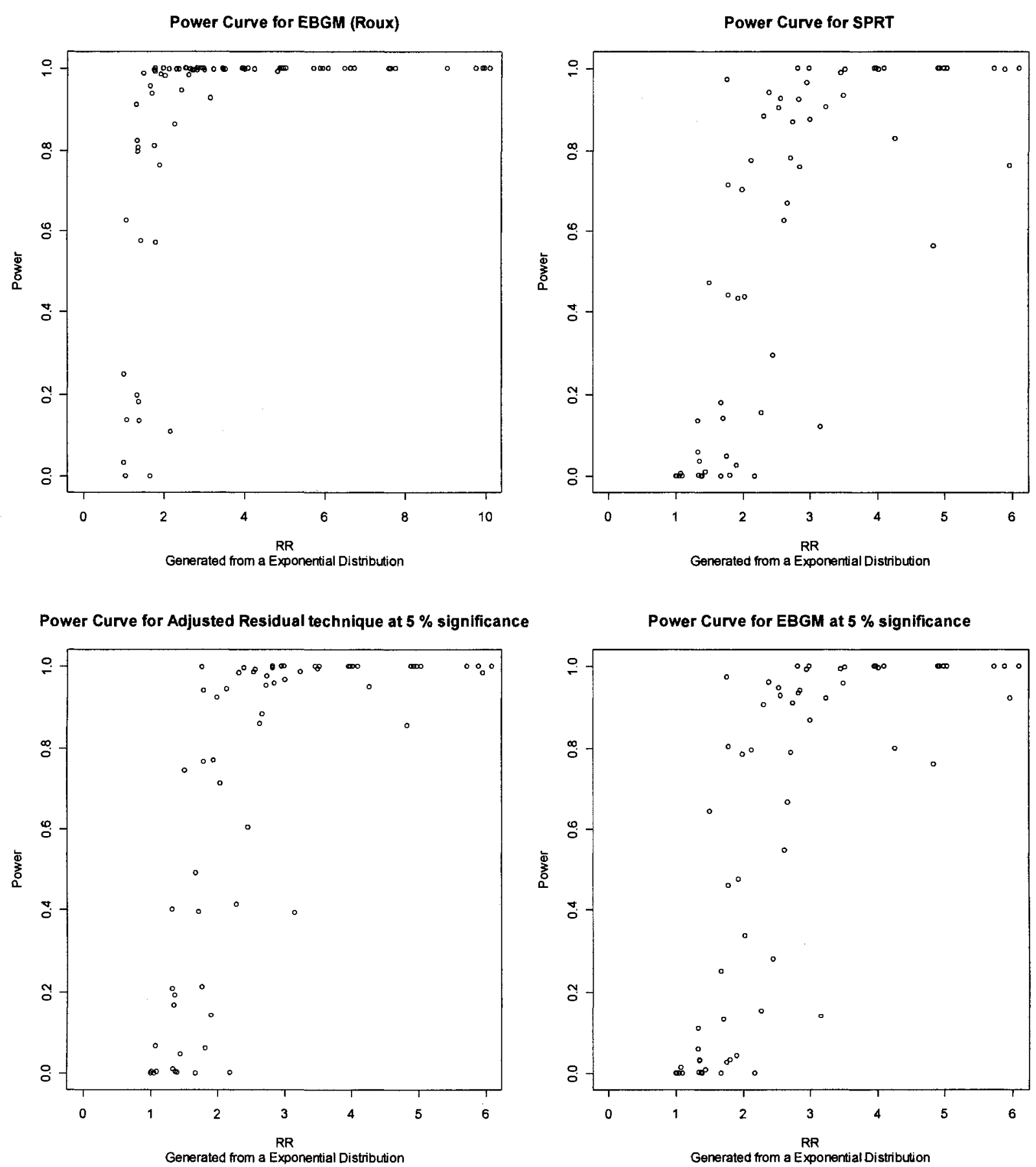

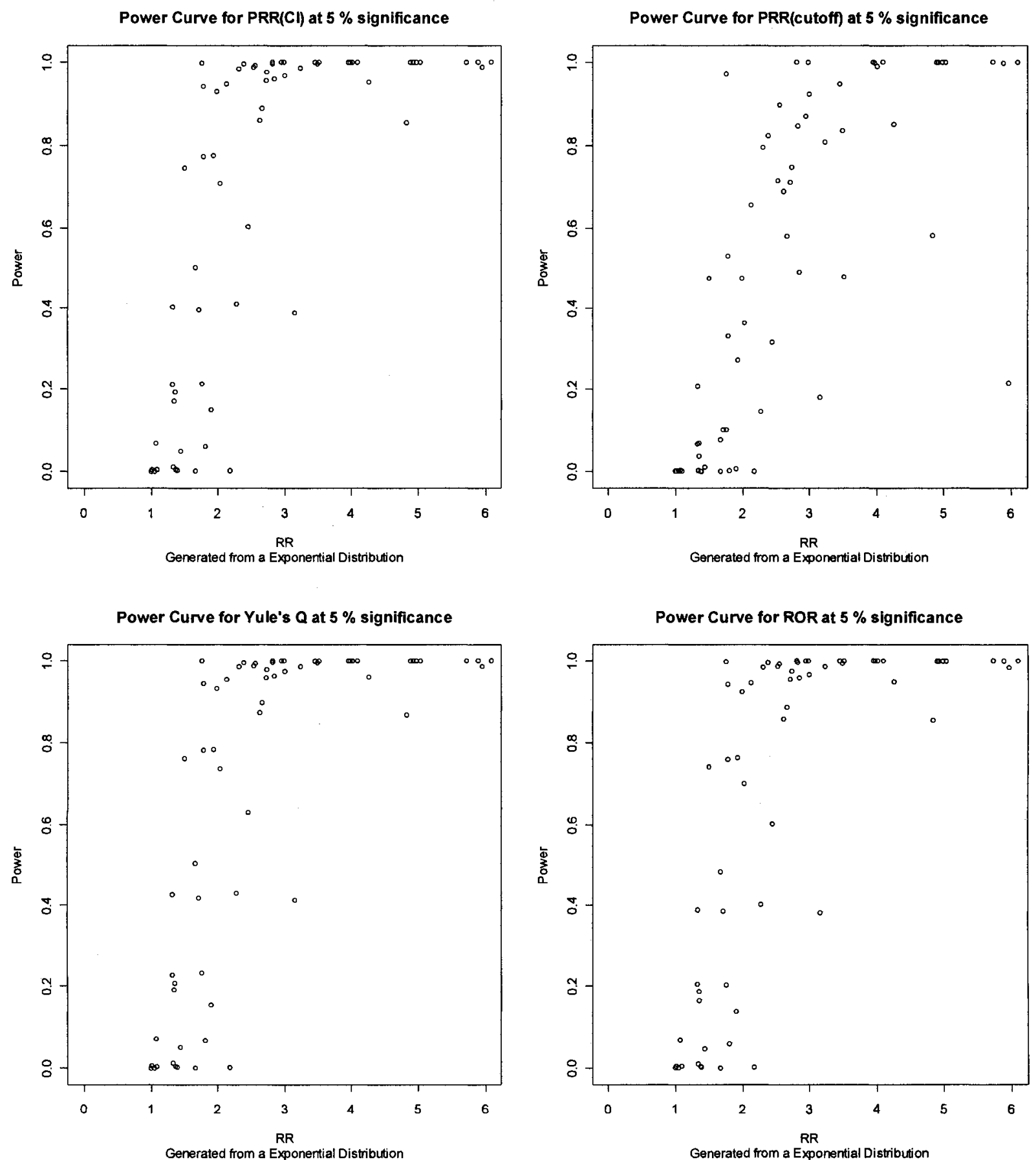

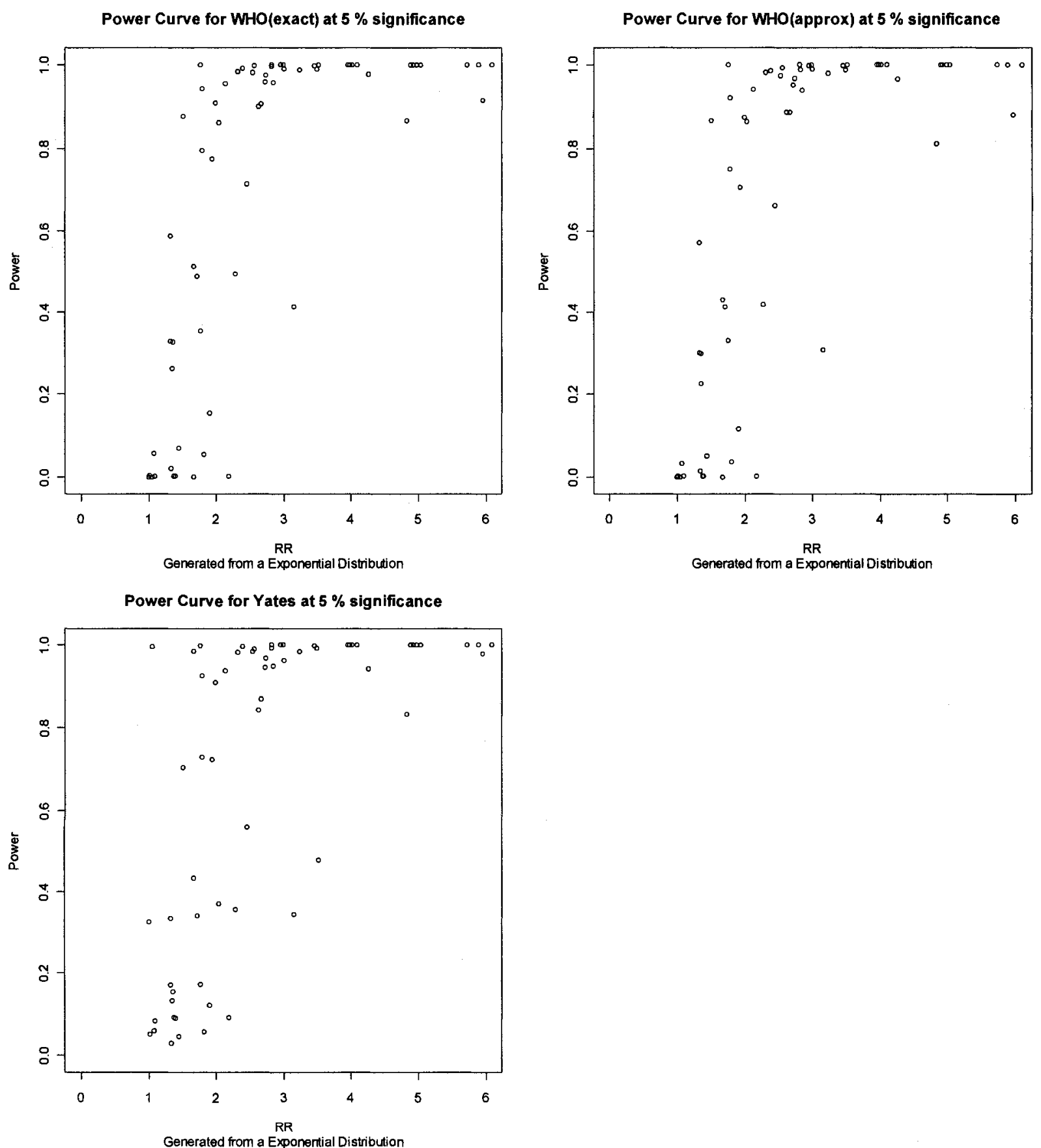

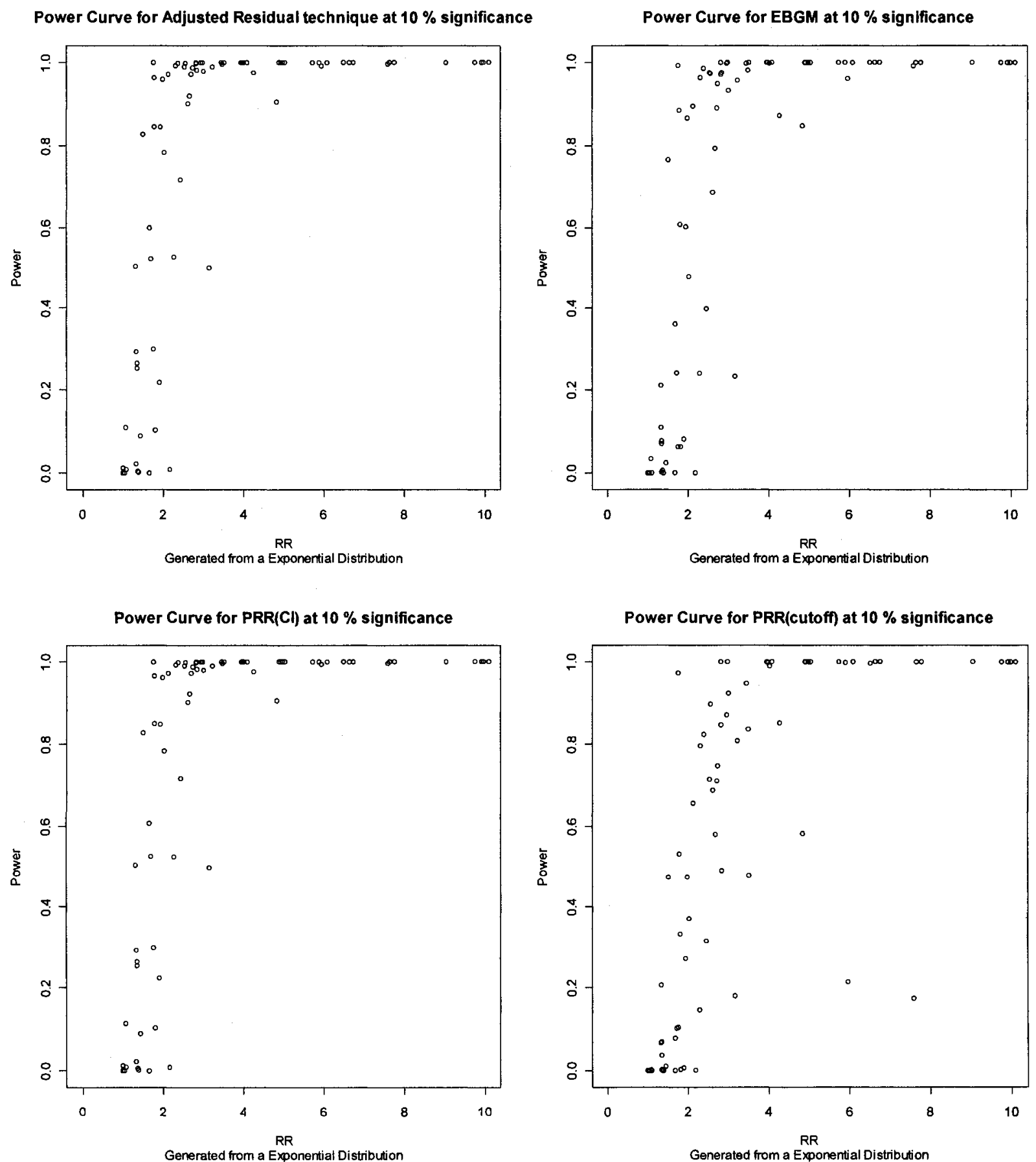

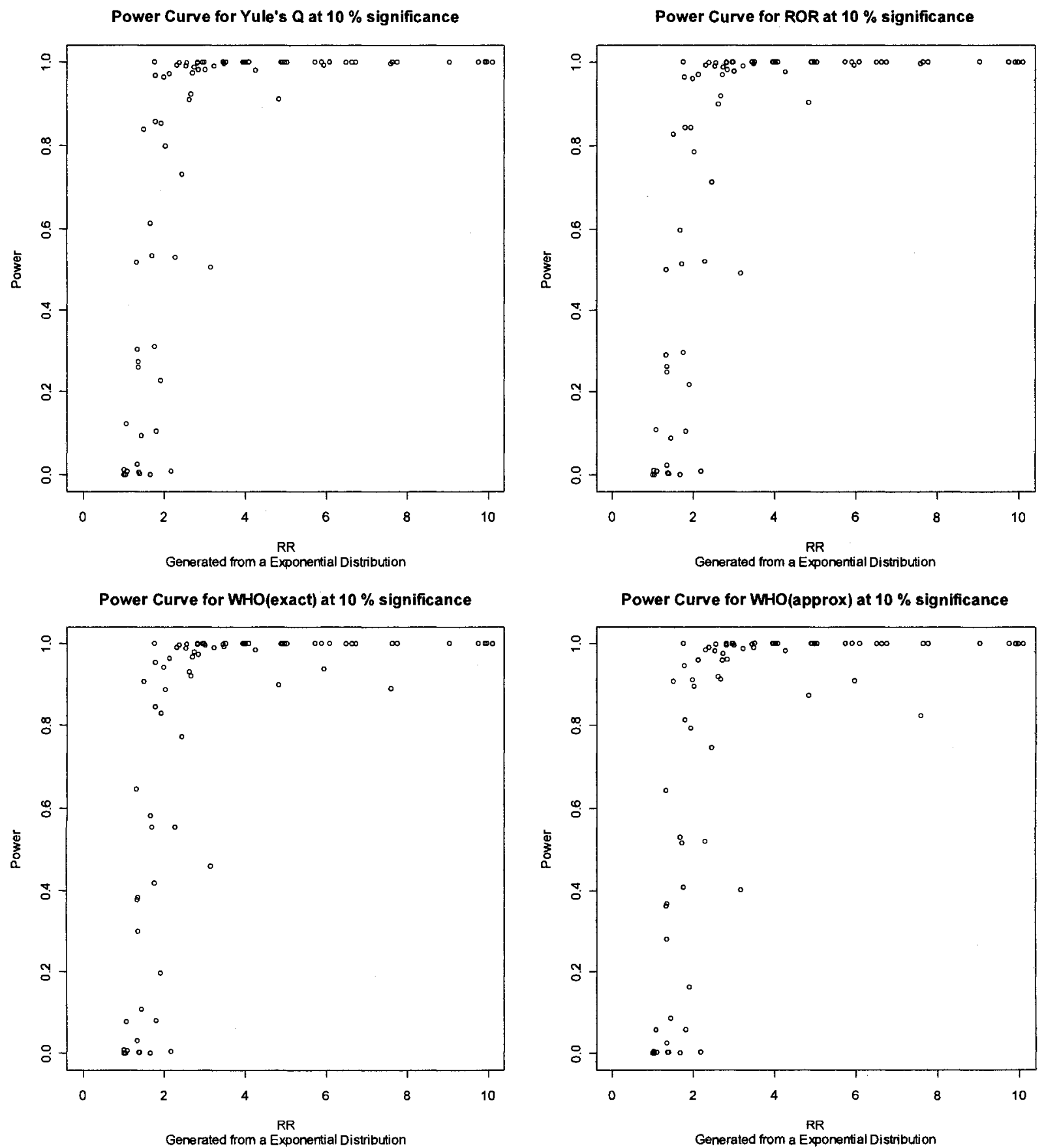


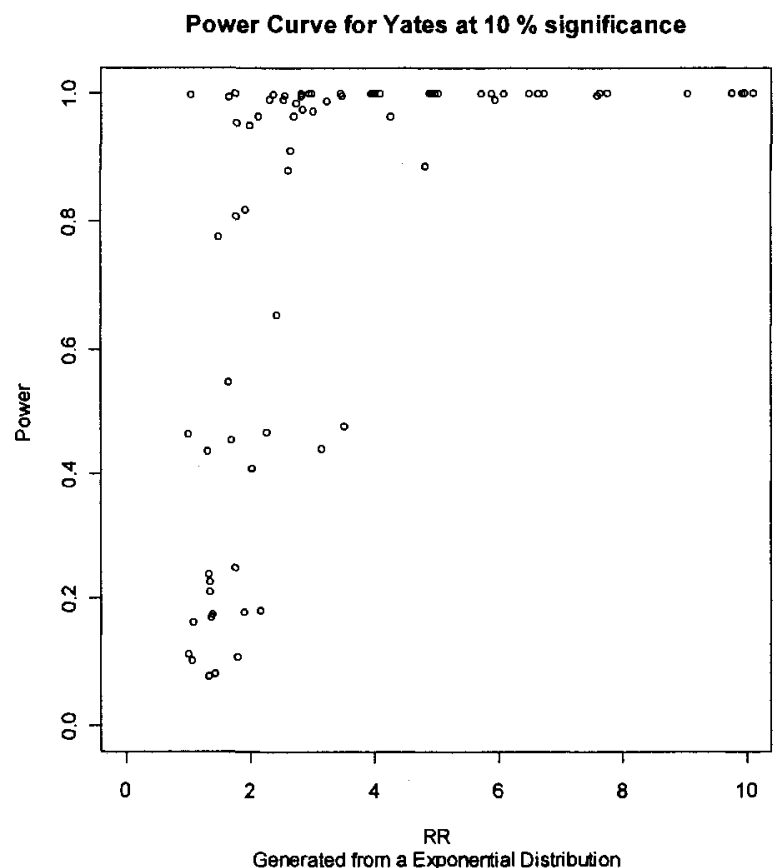



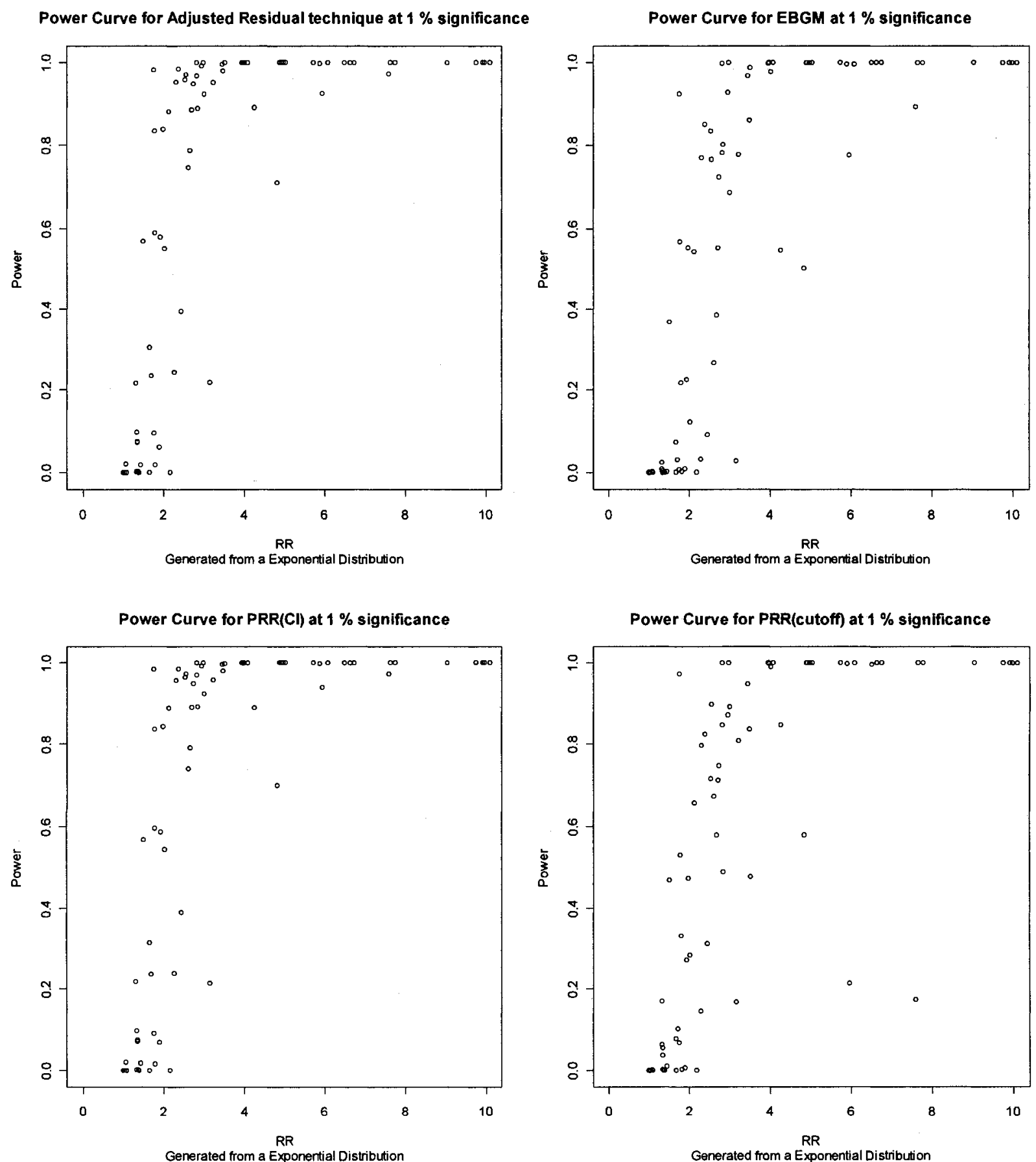

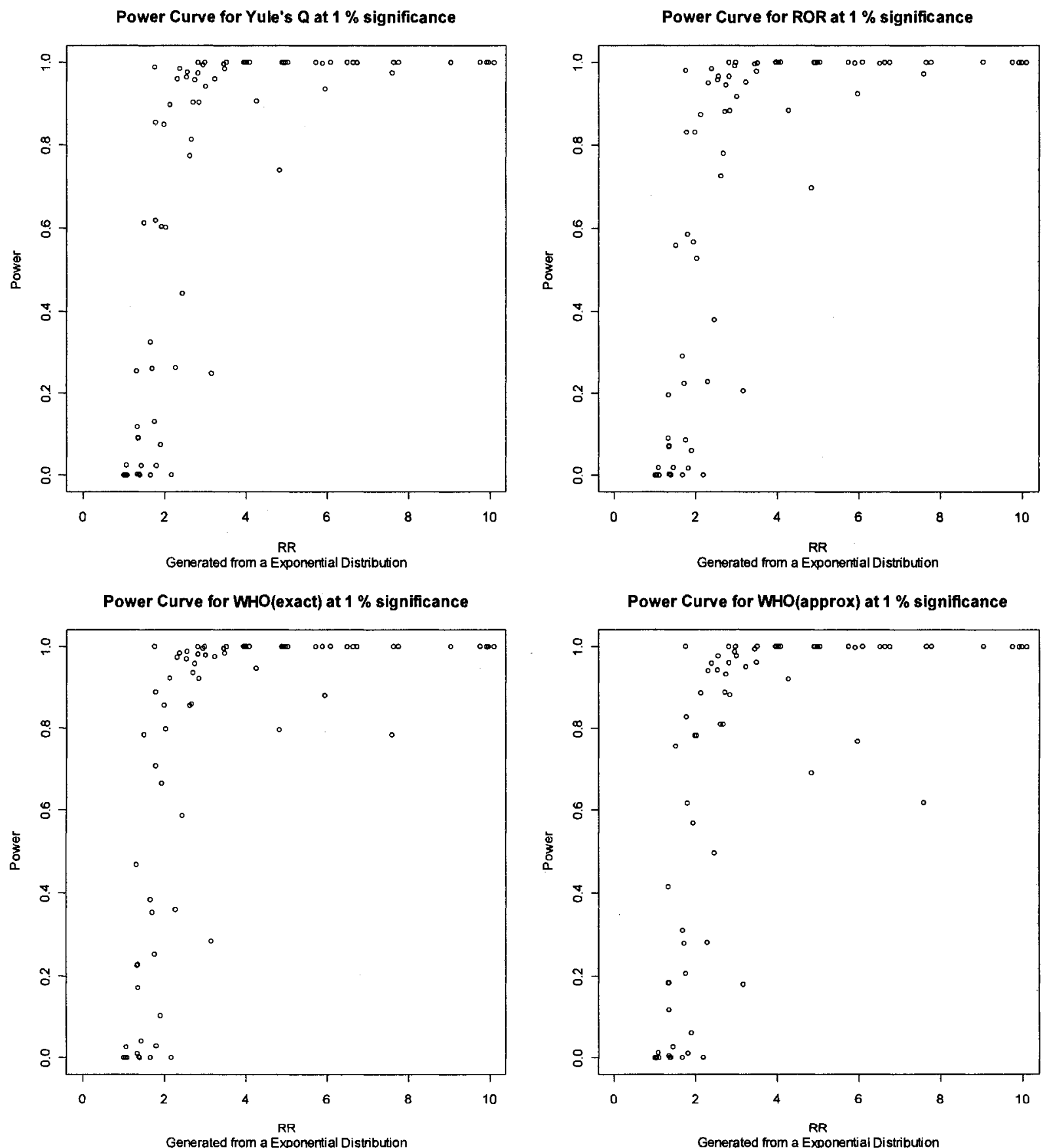


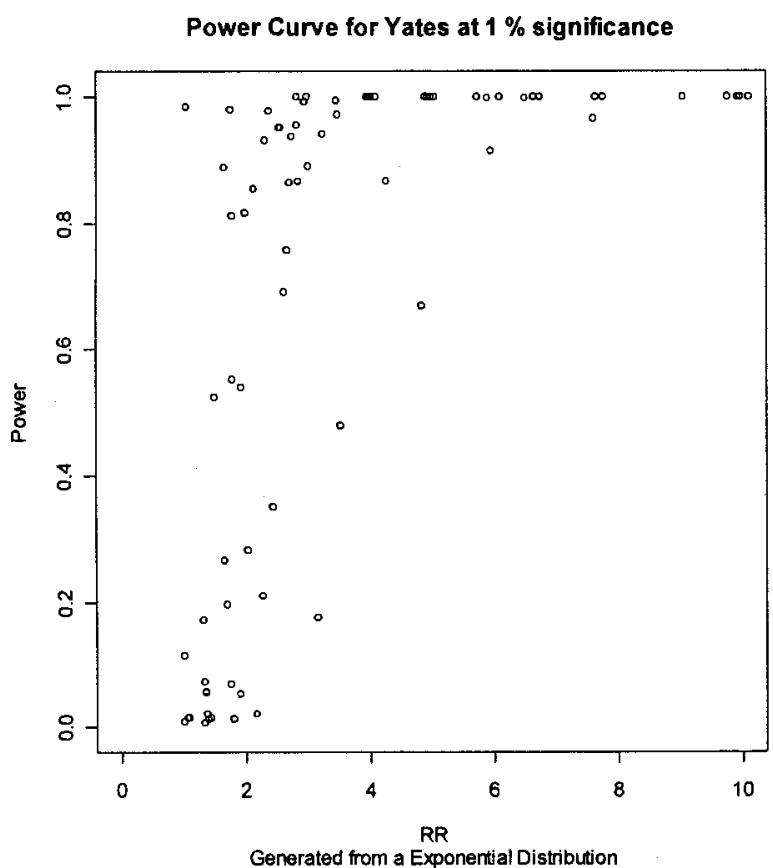


1) FDA website, $<$ http://www.fda.gov/cder/drug/infopage/COX2/default.htm $>$,

2) Agresti, A., 2002,Agresti, A. Categorical Data Analysis: New York, John Wiley.

3) Balakrishnan, N., and V. B. Nevzorov, 2003,Balakrishnan, N., and V. B. Nevzorov A Primer on Statistical Distributions: New Jersey, John Wiley \& Sons Inc..

4) Bate, A., 2007, Bayesian confidence propagation neural network: Drug Saf, v. 30, no. 7, p. 623-625.

5) Bate, A., and I. R. Edwards, 2006, Data mining in spontaneous reports: Basic Clin.Pharmacol.Toxicol., v. 98, no. 3, p. 324-330.

6) Bate, A., M. Lindquist, I. R. Edwards, S. Olsson, R. Orre, A. Lansner, and R. M. De Freitas, 1998, A Bayesian neural network method for adverse drug reaction signal generation: Eur.J.Clin.Pharmacol., v. 54, no. 4, p. 315-321.

7) Bate, A., M. Lindquist, I. R. Edwards, and R. Orre, 2002, A data mining approach for signal detection and analysis: Drug Saf, v. 25, no. 6, p. 393-397.

8) Bombardier, C. et al., 2000, Comparison of upper gastrointestinal toxicity of rofecoxib and naproxen in patients with rheumatoid arthritis. VIGOR Study Group: N.Engl.J.Med., v. 343, no. 21, p. 1520-8, 2.

9) Cleveland, W. S., 1979, Robust Locally Weighted Regression and Smoothing Scatterplots: Journal of the American Statistical Association, v. 74, p. 829-836. 
10) D'Este, G. M., 1981, A Morgenstern-type bivariate gamma distribution: Biometrika, v. 68 , no. 1, p. $339-340$.

11) Dumouchel, W., 1999, Bayesian Data Mining in Large Frequency Tables, with an Application to the FDA Spontaneous Reporting System: The American Statistician, v. 53 , p. $177-190$.

12) Dumouchel,W, C Volinsky, T Johnson, C Cortes, D Pregibon. Squashing Flat Files Flatter. Proc.KDD 1999 , 6-15. 1999. San Diego, AMC Press.

13) Eklund, P., L. Kallin, and T. Riissanen, 2000, Fuzzy Systems: Report, p. 27-32.

14) Evans, S., 2003, Sequential probability ratio tests applied to public health problems: Controlled Clinical Trials, v. 24, p. 67S.

15) Evans, S. J., P. C. Waller, and S. Davis, 2001, Use of proportional reporting ratios (PRRs) for signal generation from spontaneous adverse drug reaction reports: Pharmacoepidemiol.Drug Saf, v. 10, no. 6, p. 483-486.

16) Gould, A. L., 2003, Practical pharmacovigilance analysis strategies: Pharmacoepidemiol.Drug Saf, v. 12, no. 7, p. 559-574.

17) Greenland, S., and K. J. Rothman, 2008,Greenland, S., and K. J. Rothman Modern Epidemiology: Philadelphia, Lippincott-Raven.

18) Hauben, M., D. Madigan, C. M. Gerrits, L. Walsh, and E. P. van Puijenbroek, 2005, The Role of Data Mining in Pharmacovigilance: Expert Opinion on Drug Safety, v. 4, no. 5 , p. $929-948$. 
19) Kotz, S., N. Balakrishnan, and N. L. Johnson, 2000,Kotz, S., N. Balakrishnan, and N. L. Johnson Continuous Multivariate Distributions: Models and Applications: New York, John Wiley \& Sons Inc..

20) Orre, R., A. Lanser, A. Bate, and M. Lindquist, 2000, Bayesian neural networks with confidence estimations applied to data mining: Computational Statistics and Data Analysis, v. 34, p. 473-493.

21) Rothman, K. J., S. Lanes, and S. T. Sacks, 2004, The reporting odds ratio and its advantages over the proportional reporting ratio: Pharmacoepidemiol.Drug Saf, v. 13, no. 8 , p. 519-523.

22) Roux,E, F Thiessard, A Fourrier, B Begaud, P Tubert-Bitter. Spontaneous Reporting System Modelling for Data Mining Methods Evaluation in Pharmacovigilance. AIME Workshop on Intelligent Data Analysis in Medicine and Pharmacology (IDAMAP). 2003.

Ref Type: Conference Proceeding

23) Roux, E., F. Thiessard, A. Fourrier, B. Begaud, and P. Tubert-Bitter, 2005, Evaluation of statistical association measures for the automatic signal generation in pharmacovigilance: IEEE Trans.Inf.Technol.Biomed., v. 9, no. 4, p. 518-527.

24) Solomon, D. H., S. Schneeweiss, R. Levin, and J. Avorn, 2004, Relationship between COX-2 specific inhibitors and hypertension: Hypertension, v. 44, no. 2, p. 140-145.

25) Szarfman, A., S. G. Machado, and R. T. O'Neill, 2002, Use of screening algorithms and computer systems to efficiently signal higher-than-expected combinations of 
drugs and events in the US FDA's spontaneous reports database: Drug Saf, v. 25, no. 6, p. 381-392.

26) Tubert, P., and B. Begaud, 1991, Random models for margins of a $2 \times 2$ contingency table and application to pharmacovigilance: Stat.Med., v. 10, no. 6, p. 991-999.

27) Tubert, P., B. Begaud, F. Haramburu, and J. C. Pere, 1991, Spontaneous reporting: how many cases are required to trigger a warning?: Br.J.Clin.Pharmacol., v. 32, no. 4, p. 407-408.

28) van Puijenbroek, E. P., A. Bate, H. G. Leufkens, M. Lindquist, R. Orre, and A. C. Egberts, 2002, A comparison of measures of disproportionality for signal detection in spontaneous reporting systems for adverse drug reactions: Pharmacoepidemiol.Drug Saf, v. 11 , no. 1 , p. 3-10.

29) Wald, A., 1945, Sequential Tests of Statistical Hypotheses: The Annals of Mathematical Statistics, v. 16, no. 2, p. 117-186.

30) Waller, P., P. E. van, A. Egberts, and S. Evans, 2004, The reporting odds ratio versus the proportional reporting ratio: 'deuce': Pharmacoepidemiol.Drug Saf, v. 13, no. 8, p. $525-526$.

31) Whelton, A., W. B. White, A. E. Bello, J. A. Puma, and J. G. Fort, 2002, Effects of celecoxib and rofecoxib on blood pressure and edema in patients $>$ or $=65$ years of age with systemic hypertension and osteoarthritis: Am.J.Cardiol., v. 90, no. 9, p. 959963.

32) Yates, F., 1934, Contingency table involving small numbers and the $\chi^{2}$ test: Journal of the Royal Statistical Society, v. 1, Supplement, p. 217-235. 